\title{
THE REGULARISATION OF THE $N$-WELL PROBLEM BY FINITE ELEMENTS AND BY SINGULAR PERTURBATION ARE SCALING EQUIVALENT IN TWO DIMENSIONS
}

\author{
ANDREW LORENT ${ }^{1}$
}

\begin{abstract}
Let $K:=S O(2) A_{1} \cup S O(2) A_{2} \ldots S O(2) A_{N}$ where $A_{1}, A_{2}, \ldots, A_{N}$ are matrices of nonzero determinant. We establish a sharp relation between the following two minimisation problems in two dimensions. Firstly the $N$-well problem with surface energy. Let $p \in[1,2], \Omega \subset \mathbb{R}^{2}$ be a convex polytopal region. Define

$$
I_{\epsilon}^{p}(u)=\int_{\Omega} d^{p}(D u(z), K)+\epsilon\left|D^{2} u(z)\right|^{2} \mathrm{~d} L^{2} z
$$

and let $A_{F}$ denote the subspace of functions in $W^{2,2}(\Omega)$ that satisfy the affine boundary condition $D u=F$ on $\partial \Omega$ (in the sense of trace), where $F \notin K$. We consider the scaling (with respect to $\epsilon$ ) of

$$
m_{\epsilon}^{p}:=\inf _{u \in A_{F}} I_{\epsilon}^{p}(u) .
$$
\end{abstract}

Secondly the finite element approximation to the $N$-well problem without surface energy. We will show there exists a space of functions $\mathcal{D}_{F}^{h}$ where each function $v \in \mathcal{D}_{F}^{h}$ is piecewise affine on a regular (non-degenerate) $h$-triangulation and satisfies the affine boundary condition $v=l_{F}$ on $\partial \Omega$ (where $l_{F}$ is affine with $D l_{F}=F$ ) such that for

$$
\alpha_{p}(h):=\inf _{v \in \mathcal{D}_{F}^{h}} \int_{\Omega} d^{p}(D v(z), K) \mathrm{d} L^{2} z
$$

there exists positive constants $\mathcal{C}_{1}<1<\mathcal{C}_{2}$ (depending on $A_{1}, \ldots, A_{N}, p$ ) for which the following holds true

$$
\mathcal{C}_{1} \alpha_{p}(\sqrt{\epsilon}) \leq m_{\epsilon}^{p} \leq \mathcal{C}_{2} \alpha_{p}(\sqrt{\epsilon}) \text { for all } \epsilon>0
$$

Mathematics Subject Classification. $74 \mathrm{~N} 15$.

Received June 7, 2007. Revised January 15, 2008.

Published online June 24, 2008.

\section{INTRODUCTION}

The main goal of this paper is to show the equivalence in two dimensions (in the sense of scaling) of two different regularisations of a non-convex variational problem that forms a model of crystalline microstructure,

Keywords and phrases. Two wells, surface energy.

1 MIS MPG, Inselstrasse 22, 04103 Leipzig, Germany. andrew.lorent@sns.it 
specifically regularisation by second order gradients (otherwise known as singular perturbation) and regularisation by discretation via finite elements.

We focus on the simplest problem with non-trivial symmetries, the $N$-well problem in two dimensions. To set the scene let us take the Ball-James [3,4], Chipot-Kinderlehrer [6] approach to crystal microstructure. We have an energy function $\mathcal{I}$ on the space of deformations $u: \Omega \subset \mathbb{R}^{3} \rightarrow \mathbb{R}^{3}$ which has the form

$$
\mathcal{I}(u)=\int_{\Omega} W(D u(x)) \mathrm{d} L^{2} x
$$

where $W$ is the stored energy density function that describes the various properties of the material. The function $W$ has its minimum on a set of matrices known as the wells

$$
K=S O(3) A_{1} \cup S O(3) A_{2} \ldots S O(3) A_{N} .
$$

Roughly speaking the $A_{1}, A_{2}, \ldots, A_{N}$ are symmetry related and represent the lattice states of the material.

Since $w$ must be invariant with respect to rotation of the ambient space the wells $K$ must have form (1.2). Functional $\mathcal{I}$ is minimised over the space of functions that have affine boundary condition $F \notin K$.

A key point is that functional $\mathcal{I}$ is not weakly lower semi-continuous. Minimising sequences form finer and finer oscillations, as is to be expected in any model designed to capture properties of microstructure.

Surprisingly for certain choices of $K$ of the form (1.2) in two or three dimensions, the quasiconvex hull (see [27] for precise definitions and more information) of $K$ (which we denote $K^{q c}$ ) is sufficiently rich to allow for the existence of $F \in K^{q c} \backslash K$ for which there exists an exact minimiser of $\mathcal{I}$ over a space of function with boundary conditions $F$. Specifically if $K=S O(2) \cup S O(2) H$ where $H=\left(\begin{array}{ll}\lambda & 0 \\ 0 & \mu\end{array}\right)$ and $\mu \lambda \geq 1$ [35], or $K=S O(2) A_{1} \cup S O(2) A_{2} \ldots S O(2) A_{k}$ where $A_{1}, A_{2}, \ldots, A_{k}$ satisfy a certain condition [14], or if $K$ are the so call cubic to tetragonal wells $K=S O(3) U_{1} \cup S O(3) U_{2} \cup S O(3) U_{3}$ where for $\lambda>1$

$$
U_{1}=\left(\begin{array}{ccc}
\lambda^{2} & 0 & 0 \\
0 & \frac{1}{\lambda} & 0 \\
0 & 0 & \frac{1}{\lambda}
\end{array}\right), U_{2}=\left(\begin{array}{ccc}
\frac{1}{\lambda} & 0 & 0 \\
0 & \lambda^{2} & 0 \\
0 & 0 & \frac{1}{\lambda}
\end{array}\right) \text { and } U_{3}=\left(\begin{array}{ccc}
\frac{1}{\lambda} & 0 & 0 \\
0 & \frac{1}{\lambda} & 0 \\
0 & 0 & \lambda^{2}
\end{array}\right)
$$

[15], then in these cases there is an exact minimiser to $\mathcal{I}$ for some $F \in K^{q c} \backslash K$. This follows from work of MüllerŚverák [29,30], Sychev [33,34], Kirchheim [19,20] and Conti-Dolzmann-Kirchheim [11], see also DacorognaMarcellini [12] for a different approach to some related problems. The approach of Müller-Šverák uses the theory of "convex integration" (denoted by CI from this point) developed by Gromov, it is one of the simplest results of the theory.

Functional $\mathcal{I}$ does not constrain oscillations of the gradient, it does not give a length scale or any restriction on the fine geometry of the microstructure. For many materials, the observed length scale of the microstructure is many orders larger than the atomic scale and for these materials functional $\mathcal{I}$ is only a first approximation. To overcome this the following adaption of the functional $\mathcal{I}$ is commonly made, see [27], Section 6 ,

$$
\mathcal{I}_{\epsilon}(u)=\int_{\Omega} W(D u(z))+\epsilon\left|D^{2} u(z)\right|^{2} \mathrm{~d} L^{2} z .
$$

Roughly speaking this is a regularisation of $\mathcal{I}$ that constrains the minimiser $u$ of $\mathcal{I}$ to have less than $M$ interfaces when typically $M$ will be a negative power of $\epsilon$ that depends on $K$ and $W$. For example if we take $K=S O(2) \cup S O(2) H$ (with $\operatorname{det}(H)=1$ ) and $W(\cdot) \sim d(\cdot, K)$ then using the characterisation of Šverák [35] (as will be explained later) we have the upper bound of $\inf \mathcal{I}_{\epsilon} \leq c \epsilon^{\frac{1}{6}}$. Let $v(z):=u(\sqrt{\epsilon} z) \epsilon^{-\frac{1}{2}}$, then $\int_{\epsilon^{-\frac{1}{2} \Omega}}\left|D^{2} v\right|^{2} \leq m_{\epsilon} \epsilon^{-1} \leq c \epsilon^{-\frac{5}{6}}$. Now $v$ satisfies the elliptic Euler Lagrange equation $\operatorname{div} D W(D v)+\Delta^{2} v=0$ which by standard elliptic regularity means $D v$ is Holder with Holder exponent $O(1)$, thus each interface running through $\epsilon^{-\frac{1}{2}} \Omega$ contributes $O\left(\epsilon^{-\frac{1}{2}}\right)$ to $\int_{\epsilon^{-\frac{1}{2}} \Omega}\left|D^{2} v\right|^{2}$ so we have at most $M \leq c \epsilon^{\frac{1}{2}} \epsilon^{-\frac{5}{6}}=c \epsilon^{-\frac{1}{3}}$ such interfaces. 
There have been a number of studies of simplified versions of functional $\mathcal{I}_{\epsilon}[8,22,26]$. However these works focus on the case where $\mathcal{I}_{\epsilon}$ acts on scalar functions and the wells of $\mathcal{I}_{\epsilon}$ are given by two matrices. In this case (scaling) sharp upper and lower bounds have been proved. For functional with wells that have rotational invariance, i.e. of the form (1.2), nothing is known about the energy of minimisers.

Another way to constrain oscillation in the gradient is to minimise $\mathcal{I}$ directly over the space of functions that are piecewise affine on a $h$ sized triangular grid. This is known as the finite element approximation of $\mathcal{I}$. There have been many studies of finite element approximations to functionals of the form $\mathcal{I}$, again for the simplified case where the wells are given by sets of two or three matrices [5,7,23,24].

Our main achievement in this paper is to show that for the specific stored energy function $W(\cdot) \sim d^{p}(\cdot, K)$ (for some $p \in[1,2]$ ) these two regularisations are scaling equivalent.

For the case where the wells of $\mathcal{I}$ are given by sets of two or three matrices (and $W(\cdot) \sim d(\cdot, K)$ ) it is possible to calculate the scaling of the energy of $\mathcal{I}_{\epsilon}$ and the scaling of the energy of the finite element approximation to $\mathcal{I}$ $[7,23]$. To be more specific given matrices $A, B$ with $\operatorname{rank}(A-B)=1$ using methods from [7] it can be shown that for wells $K_{1}=\{A, B\}$ the functional $\mathcal{I}^{1}$ minimised over the space of functions that are piecewise affine on a $h$-sized triangular grid ${ }^{1}$ and have affine boundary condition $F_{0}=\mu_{0} A+\left(1-\mu_{0}\right) B$ (for some $\mu_{0} \in(0,1)$ ) scales like $\sqrt{h}$. Strictly speaking the functional studied in [7] acts on scalar functions but the method works for the case stated above with minor modifications. In [23] three rank-1 connected matrices were considered, expanding on the methods of [7] it was shown in [23] that if functional $\mathcal{I}^{2}$ has wells $K_{2}=\left\{\left(\begin{array}{cc}-1 & 0 \\ 0 & -1\end{array}\right),\left(\begin{array}{cc}-1 & 0 \\ 0 & 1\end{array}\right),\left(\begin{array}{ll}1 & 0 \\ 0 & 0\end{array}\right)\right\}$ (and $W(\cdot) \sim d\left(\cdot, K_{2}\right)$ ) then over the space of piecewise affine functions with boundary condition $F_{1}=\left(\begin{array}{ll}0 & 0 \\ 0 & 0\end{array}\right)$ the energy of functional $\mathcal{I}^{2}$ scales like $h^{\frac{1}{3}}$. Using very similar methods to [7] and [23] it is possible to show that functionals $\mathcal{I}_{\epsilon}^{a}:=\int_{\Omega} \mathrm{d}\left(D u, K_{a}\right)+\epsilon\left|D^{2} u\right|^{2}$ for $a=1,2$ are such that their energy scales like inf $\mathcal{I}_{\epsilon}^{1} \sim \epsilon^{\frac{1}{4}}$ and $\inf \mathcal{I}_{\epsilon}^{2} \sim \epsilon^{\frac{1}{6}}$.

Thus for functionals whose wells are given by sets of two or three matrices our main theorem is of no interest, for in these cases we can calculate the scaling and it can be seen instantly that the energy of functional $\mathcal{I}^{a}$ taken over a space of function that are piecewise affine on a grid of size $\sqrt{\epsilon}$ scales in the same way as the energy of functional $\mathcal{I}_{\epsilon}^{a}$. The point of this paper is that we study functional $\mathcal{I}_{\epsilon}$ with wells

$$
K=S O(2) A_{1} \cup S O(2) A_{2} \ldots S O(2) A_{N}
$$

and for these wells the scaling of the energy of $\mathcal{I}_{\epsilon}$ and the scaling of the energy of $\mathcal{I}$ over the space of piecewise affine functions are completely unknown. In this case our main theorem tells us that these two problem, one discrete and one continuous, are scaling equivalent.

\subsection{Background and statement of main result}

To state our theorem we need to give some background. Given a polytopal region $\Omega$ and some small constant $\varsigma \in(0,1)$ we say a collection of disjoint triangles $\left\{\tau_{i}\right\}$ is an $(h, \varsigma)$-triangulation of $\Omega$ if $\bigcup_{i} \overline{\tau_{i}}=\Omega$ and every triangle $\tau_{i}$ contains a ball of radius $\varsigma h$ and has diameter less than $\varsigma^{-1} h$. Given $w \in S^{1}$ we denote by $\triangle_{h}^{\varsigma}(w)$ the set of regular triangulations with respect to axis $\langle w\rangle, w^{\perp}$ axis, by this we mean every triangle $\tau_{i}$ of distance $\varsigma^{-1} h$ from $\partial \Omega$ is a right angle triangle with sides parallel to $\langle w\rangle, w^{\perp}$. Finally we let $\mathcal{F}_{F}^{\varsigma, h}(w)$ denote the space of functions that are piecewise affine on some triangulation in $\triangle_{h}^{\varsigma}(w)$ and satisfy the affine boundary condition $u=l_{F}$ on $\partial \Omega$, where $l_{F}$ is a fixed affine function with $D l_{F}=F$.

Given two connected subsets of matrices $M, N \subset M^{2 \times 2}$ we say $M$ and $N$ are rank-1 connected if and only if there exists $A \in M$ and $B \in N$ and $v \in S^{1}$ such that $A v=B v$. The set of rank-1 directions connecting $M, N$ are the set of vectors $v \in S^{1}$ satisfying $A v=B v$ for some $A \in M, B \in N$.

\footnotetext{
${ }^{1}$ Whose edges are not parallel to the rank-1 connections between $A$ and $B$.
} 
For given triangulation $\left\{\tau_{i}\right\}$ and function $u \in \mathcal{F}_{F}^{\varsigma, h}(w)$ and triangle $\tau_{i}$ we define the neighbouring gradients by

$$
N_{i}(u)= \begin{cases}\left\{D u_{\left\lfloor\tau_{j}\right.}: \overline{\tau_{j}} \cap \overline{\tau_{i}} \neq \emptyset\right\} & \text { for } i \text { such that } \overline{\tau_{i}} \cap \partial \Omega=\emptyset \\ \left\{D u_{\left\lfloor\tau_{j}\right.}: \overline{\tau_{j}} \cap \overline{\tau_{i}} \neq \emptyset\right\} \cup\{F\} & \text { for } i \text { such that } \overline{\tau_{i}} \cap \partial \Omega \neq \emptyset .\end{cases}
$$

And for $u \in \mathcal{F}_{F}^{\varsigma, h}$ we define the jump triangles by

$$
J(u):=\left\{i: \exists A, B \in N_{i}(u) \text { such that }|A-B|>\varsigma^{-1}\right\} .
$$

Let $\sigma$ be the minimum of the absolute values of the eigenvalues of $A_{1}, \ldots, A_{N}$. Let $w_{1} \in S^{1}$ be such that for some $w_{2} \in w_{1}^{\perp}$ we have that $w_{1}, w_{2}, \frac{w_{1}-w_{2}}{\left|w_{1}-w_{2}\right|}$ are not in the set of rank-1 directions connecting $S O(2) A_{i}$ to $S O(2) A_{j}$ for any $i \neq j$, let $\varsigma \in\left(0,10^{-1} \sigma\right)$ we define function space

$$
\mathcal{D}_{F}^{\varsigma, h}\left(w_{1}\right):=\left\{v \in \mathcal{F}_{F}^{\varsigma, h}\left(w_{1}\right): \sum_{i \in J(v)} \sum_{M \in N_{i}(v)}\left|D v_{\left\lfloor\tau_{i}\right.}-M\right|^{2} \leq \varsigma^{-1} h^{-2} \int_{\Omega} d^{p}(D v, K)\right\} .
$$

When there is no ambiguity we will denote these function spaces just as $\mathcal{F}_{F}^{\varsigma, h}$ or $\mathcal{D}_{F}^{\varsigma, h}$. Clearly inf $v_{v \in \mathcal{F}_{F}^{\varsigma, h}} I_{0}^{p}(v) \leq$ $\inf _{v \in \mathcal{D}_{F}^{\varsigma, h}} I_{0}^{p}(v)$, the main reason for introducing function space $\mathcal{D}_{F}^{\varsigma, h}$ is that with our methods we can not show the sharpness of the lower bound

$$
\inf _{v \in \mathcal{F}_{F}^{s, \sqrt{\epsilon}}} I_{0}^{p}(v) \leq c \inf _{v \in A_{F}} I_{\epsilon}(v)
$$

(where $A_{F}$ is the subspace of functions in $u \in W^{2,2}(\Omega)$ with $D u=F$ in the sense of trace). So instead we will prove the stronger lower bound $\inf _{v \in \mathcal{D}_{F}^{\varsigma, \sqrt{\epsilon}}} I_{0}^{p}(v) \leq c \inf _{u \in A_{F}} I_{\epsilon}(u)$ and it turns out that function space $\mathcal{D}_{F}^{\varsigma, h}$ has enough structure to allow us to show the upper bound ${ }^{2}$

$$
\inf _{u \in A_{F}} I_{\epsilon}^{p}(u) \leq c \inf _{v \in \mathcal{D}_{F}^{s, \sqrt{\epsilon}}} I_{0}^{p}(v) .
$$

Our main theorem is the following.

Theorem 1.1. Let $K:=S O(2) A_{1} \cup S O(2) A_{2} \ldots S O(2) A_{N}$ where $A_{1}, A_{2}, \ldots, A_{N} \in M^{2 \times 2}$ are matrices of non-zero determinant. Let $\sigma$ be the minimum of the absolute values of the eigenvalues of $A_{1}, \ldots, A_{N}$.

Let $w_{1} \in S^{1}$ be such that for some $w_{2} \in w_{1}^{\perp}$, the vectors $w_{1}, w_{2}, \frac{w_{1}-w_{2}}{\left|w_{1}-w_{2}\right|}$ are not in the set of rank-1 directions connecting $S O(2) A_{i}$ to $S O(2) A_{j}$ for any $i \neq j$. Let $\Omega \subset \mathbb{R}^{2}$ be a polytopal convex domain. For $p \in[1,2]$ define

$$
I_{\epsilon}^{p}(u):=\int_{\Omega} d^{p}(D u(z), K)+\epsilon\left|D^{2} u(z)\right|^{2} \mathrm{~d} L^{2} z .
$$

Let $F \notin K$ and let $A_{F}$ denote the subspace of functions in $W^{2,2}(\Omega)$ that have boundary condition $D u=F$ on $\partial \Omega$ in the sense of trace. For $\varsigma \in\left(0,10^{-1} \sigma\right)$ let function space $\mathcal{D}_{F}^{\varsigma, h}\left(w_{1}\right)$ be defined by (1.5). If we define

$$
\alpha_{p}(h):=\inf _{v \in \mathcal{D}_{F}^{s, h}\left(w_{1}\right)} I_{0}^{p}(v) \text { and } m_{\epsilon}^{p}:=\inf _{u \in A_{F}} I_{\epsilon}^{p}(u)
$$

then there are positive constants $\mathcal{C}_{1}<1<\mathcal{C}_{2}$ (depending only on $\sigma, \varsigma, p$ ) for which

$$
\mathcal{C}_{1} \alpha_{p}(\sqrt{\epsilon}) \leq m_{\epsilon}^{p} \leq \mathcal{C}_{2} \alpha_{p}(\sqrt{\epsilon}) \text { for all } \epsilon>0 .
$$

\footnotetext{
${ }^{2}$ For further explanation as to why function space $\mathcal{D}_{F}^{\varsigma, h}$ allows us to prove (1.7) where as with our methods we can not show the same inequality for the larger function space $\mathcal{F}_{F}^{\varsigma, h}$ see Section 2.2 .
} 
The point of introducing parameter $\varsigma$ into the definition of $\mathcal{D}_{F}^{\varsigma, h}$ is that we would like to use the greater flexibility it allows for a potential future improvement of our main result. To explain this further note that the definition of $\mathcal{D}_{F}^{\varsigma, h}$ gives us the inclusion

$$
\mathcal{L}_{F}^{\varsigma, h}:=\left\{v \in \mathcal{F}_{F}^{\varsigma, h}:\|D v\|_{L^{\infty}(\Omega)} \leq 4^{-1} \varsigma^{-1}\right\} \subset \mathcal{D}_{F}^{\varsigma, h}
$$

so clearly we have the upper bound (1.7) for this function space. Given the results of [28] it seems reasonable to hope that minimisers of a functional equivalent ${ }^{3}$ to $I_{\epsilon}^{p}$ for $p>1$ are $Q$-Lipschitz (for some possibly large ${ }^{4}$ Q independent of $\epsilon$ ) inside the whole domain $\Omega$. In [28] this has only been proved for $p=2$ in an interior domain. If such a result could be proved the methods of this paper would allow us to show the lower bound $\inf _{v \in \mathcal{L}_{F}^{s, h}} I_{\epsilon}^{p}(v) \leq c \inf _{u \in A_{F}} I_{\epsilon}^{p}(u)$ which together with the upper bound would imply for $p>1$ the equivalence of the scaling of $m_{\epsilon}^{p}$ to the scaling of $I_{\epsilon}^{p}$ over the space of Lipschitz finite elements. This is the principle reason for introducing parameter $\varsigma$.

In truth our main motivation for establishing Theorem 1.1 was that we hoped to use it as a tool to understanding the minimiser of $I_{\epsilon}^{p}$. To explain this further we will simplify and take $K=S O(2) \cup S O(2) H$ where $H$ is a diagonal matrix of determinant 1 and we take $p=1$.

As mentioned, nothing is known about the minimiser of the functional $I_{\epsilon}^{1}$. In particular it is completely unknown if for very small $\epsilon$ the minimiser is something like the absolute minimiser of $I_{0}$ provided by $\mathrm{CI}^{5}$. In some sense this might seem reasonable, we refer to the $\int\left|D^{2} u\right|^{2}$ term as the "surface energy" and the $\int d(D u, K)$ term as the "bulk energy", as $\epsilon \rightarrow 0$ the surface energy becomes less and less important, the main thing to be minimised is the bulk energy and of course CI solutions have zero bulk energy.

This question is best expressed by considering the scaling of $m_{\epsilon}^{1}$. An upper bound of $m_{\epsilon}^{1} \leq c \epsilon^{\frac{1}{6}}$ is provided by the standard double laminate which follows from the characterisation of the quasiconvex hull of $S O(2) \cup S O(2) H$ provided by [35], see Figure 1.

If $m_{\epsilon} \sim \epsilon^{\frac{1}{6}+\alpha}$ for $\alpha>0$ then the minimiser will have to take a very different form than the double laminate. On the other hand if $\alpha=0$ then energetically the minimiser does no better than the double laminate.

This question is important because CI solutions are important, many counter examples to natural conjectures in PDE have been achieved via CI $[13,19,31,32]$. Minimising functional $I_{\epsilon}$ is the simplest problem that constrains oscillation in some slight way where we can hope to see the effect of the existence of exact minimisers of (1.1).

In the proof of Theorem 1.1 we have to work quite hard to establish the result for $p=1$, we do so because functional $I_{\epsilon}^{1}$ is particularly clean in the sense that it is not necessary to consider laminates with "domain branching" to construct upper bounds (contrast this with the case $p=2[8,22]$ ) as such the upper bound is given by $c \epsilon^{\frac{1}{6}}$ and is domain independent.

Let $w_{1} \in S^{1}$ be such that for $w_{2} \in w_{1}^{\perp}$ we have $w_{1}, w_{2}, \frac{w_{1}-w_{2}}{\left|w_{1}-w_{2}\right|}$ do not belong to the rank-1 connections between $S O(2)$ and $S O(2) H$. If $\tilde{u} \in \mathcal{F}_{F}^{\varsigma, h}\left(w_{1}\right)$ and $\tau_{1}, \tau_{2} \in \triangle_{h}^{\varsigma}\left(w_{1}\right)$ are such that $d\left(D \tilde{u}_{\left\lfloor\tau_{1}\right.}, S O(2)\right) \approx 0$ and $d\left(D \tilde{u}_{\left\lfloor\tau_{2}\right.}, S O(2) H\right) \approx 0$, it is not too hard to see $\tau_{1}$ can not touch $\tau_{2}$, i.e. there must be a triangle $\tau_{3}$ between $\tau_{1}$ and $\tau_{2}$ for which $d\left(D u_{\left\lfloor\tau_{3}\right.}, K\right) \geq o(1)$.

For example if we have an interpolant of a laminate, and triangle $\tau_{i}$ cuts through an interface of the laminate the affine map we get from interpolating the laminate on the corners of $\tau_{i}$ will have its linear part some distance from the wells. See Figure 2.

\footnotetext{
${ }^{3}$ In order to apply the result of [28] we need a functional that is quadratic at infinity in a strong sense, but given $W(\cdot) \sim d^{p}(\cdot, K)$ it is easy to construct a function $\widetilde{W}$ such that $W=\widetilde{W}$ in a large ball $B_{R}$ and $\|\widetilde{W}-W\|_{L^{\infty}} \leq c$ while $\widetilde{W}(M)=|M|^{p}$ for any $M \notin B_{2 R}$. Defining $\widetilde{I}_{\epsilon}^{p}(v)=\int_{\Omega} \widetilde{W}(D v)+\epsilon\left|D^{2} v\right|^{2}$ we have $\left|\widetilde{I}_{\epsilon}^{p}(v)-I_{\epsilon}^{p}(v)\right| \leq c$ for any $v \in W^{2,2}(\Omega)$ so obviously the energy of $\widetilde{I}_{\epsilon}^{p}$ and $I_{\epsilon}^{p}$ scale the same way with respect to $\epsilon$ and for the case $p=2$ it is possible to apply the results of [28] to the minimiser of $\widetilde{I}_{\epsilon}^{p}$.

${ }^{4}$ Found via a compactness argument.

${ }^{5}$ We know it can not be a function $u$ with $I_{0}(u)=0$ because the result of Dolzmann-Müller [16], that any $u$ with this property and with the property that $D u$ is a BV has to be laminate.
} 

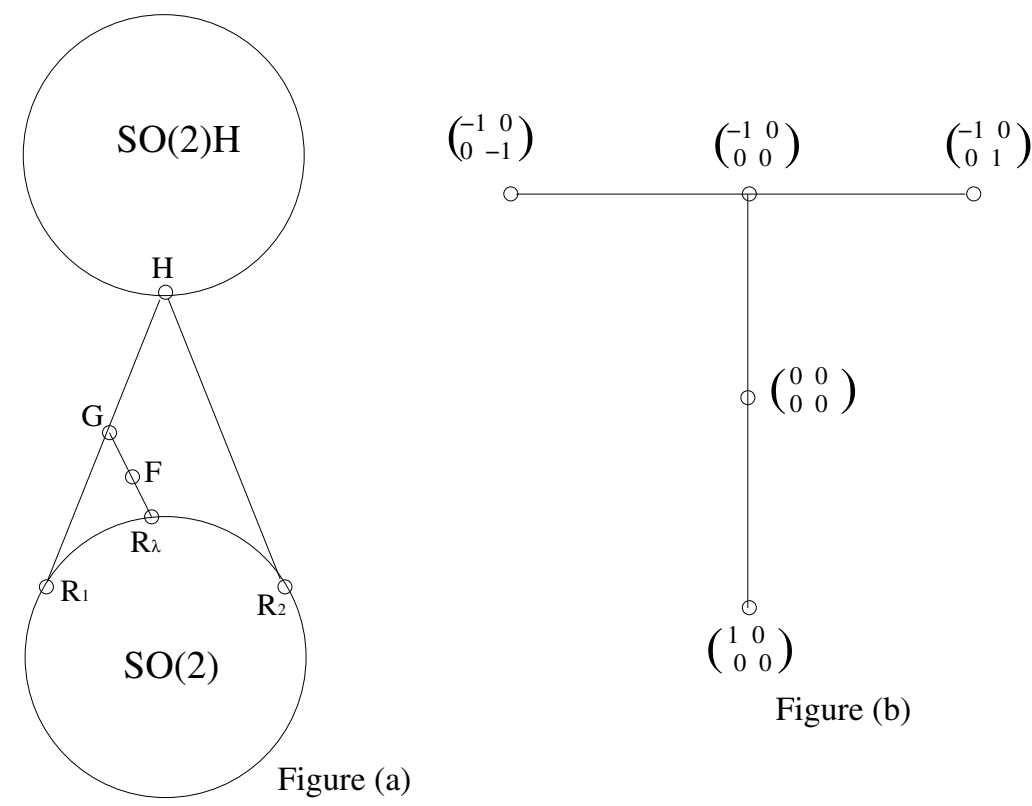

Figure (b)

FiguRE 1. Rank-1 connections between sets of matrices.

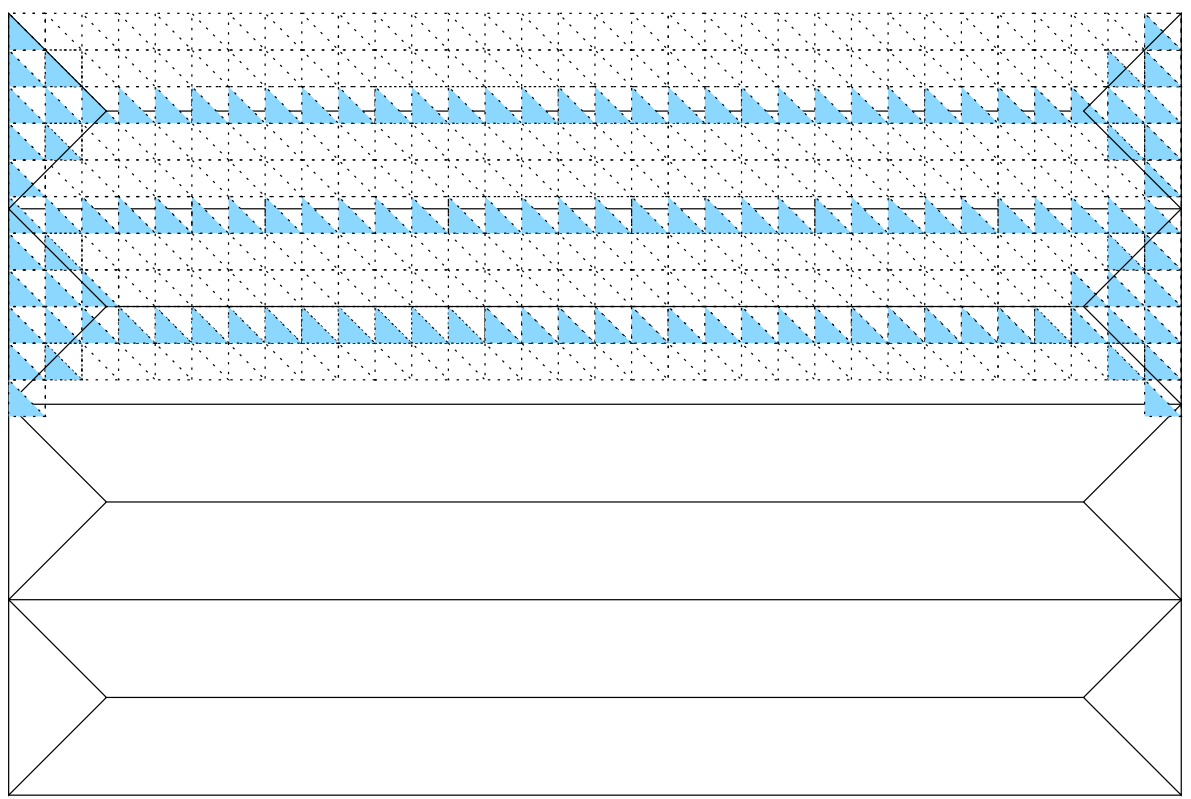

Figure 2. A finite element approximation to a laminate.

So we can not lower the energy of $I_{0}$ over $\mathcal{F}_{F}^{\varsigma, h}\left(w_{1}\right)$ by simply making a laminate type function with finer layers, there is a competition between the surface energy as given by the error contributed from the interfaces and the bulk energy which in the case of the laminate is the width of the interpolation layer. 
As mentioned for functional $\mathcal{I}^{2}$ with wells $\left\{\left(\begin{array}{cc}-1 & 0 \\ 0 & -1\end{array}\right),\left(\begin{array}{cc}-1 & 0 \\ 0 & 1\end{array}\right),\left(\begin{array}{ll}1 & 0 \\ 0 & 0\end{array}\right)\right\}$ in [23] it was shown the energy of $\mathcal{I}^{2}$ over the space $\mathcal{F}_{F_{1}}^{\varsigma, h}$ (where $F_{1}=\left(\begin{array}{ll}0 & 0 \\ 0 & 0\end{array}\right)$ ) scales like $h^{\frac{1}{3}}$. From Šverák's characterisation [35] we know the exact arrangement of rank- 1 connections between the matrices in the set $S O(2) \cup S O(2) H$ and a matrix in the interior of the quasiconvex hull of $S O(2) \cup S O(2) H$, see Figure 1a. As we can see from Figures 1a and 1b, the finite well functional $\mathcal{I}^{2}$ precisely mimics these rank-1 connections.

Conjecture 1.1. Let $K=S O(2) \cup S O(2) H$ where $H$ is a diagonal matrix with eigenvalues $\sigma, \sigma^{-1}$. Let $w_{1} \in S^{1}$ and $w_{2} \in w_{1}^{\perp}$ be such that $w_{1}, w_{2}, \frac{w_{1}-w_{2}}{\left|w_{1}-w_{2}\right|}$ are not in the set of rank-1 connections between $S O(2)$ and $S O(2) H$. Let $\Omega$ be a polytopal convex region, $\varsigma \in\left(0,10^{-1} \sigma\right)$. Given $F \in \operatorname{int}\left(K^{q c}\right)$, let function space $\mathcal{F}_{F}^{\varsigma, h}\left(w_{1}\right)$ denote the space of functions that are piecewise affine on some regular triangulation $\left\{\tau_{i}\right\} \in \triangle_{h}^{\varsigma}\left(w_{1}\right)$. There exists $c_{0}=c_{0}(\sigma, \varsigma)>0$ such that

$$
\inf _{u \in \mathcal{F}_{F}^{s, h}} I_{0}^{1}(u) \geq c_{0} h^{\frac{1}{3}} \text { for all } h>0 .
$$

So from Theorem 1.1, if Conjecture 1.1 could be proved it would imply the scaling $m_{\epsilon}^{1} \sim \epsilon^{\frac{1}{6}}$. Unfortunately even though the minimisation of $I_{0}^{1}$ over $\mathcal{F}_{F}^{\varsigma, h}$ is discrete problem, it appears to be quite hard to prove lower bounds.

\section{Sketch of the Proof}

Written out in detail, the proof of Theorem 1.1 is not short, however the basic ideas are quite simple. We give a sketch of the proof based on two lemmas that are only approximate principles, by this we mean that either we can not prove them, or only a weaker form hold true. This may be a bit unconventional, but it seems to us to be the best way to get to the heart of the matter without being flooded with details.

\subsection{Lower bound}

We focus on the case $p=1$ and take $\Omega=Q_{1}(0)$. Let $M=\left[\epsilon^{-\frac{1}{2}}\right]$. We cut the square $\Omega$ into $M^{2}$ sub-squares of side length $\frac{1}{M}$, let $c_{1}, c_{2}, \ldots, c_{M^{2}}$ be the centres of these squares. So $Q_{1}(0)=\bigcup_{i=1}^{M^{2}} \overline{Q_{\frac{1}{M}}\left(c_{i}\right)}$. Let $\mathcal{C}_{1}=\mathcal{C}_{1}(\sigma)$ be some small constant we decide on later. Now we define the "bad" squares to be $B:=\left\{i: \int_{Q_{\frac{1}{M}}\left(c_{i}\right)}\left|D^{2} u\right|^{2} \geq \mathcal{C}_{1}\right\}$. Approximate principle 1. For any $i \in\left\{1,2, \ldots, M^{2}\right\} \backslash B$ define $v_{i}(z)=u\left(c_{i}+\frac{z}{M}\right) M$ we have that there exists affine function $L_{i}$ with $D L_{i} \in K$ such that

$$
\left\|v_{i}-L_{i}\right\|_{L^{\infty}\left(Q_{1}(0)\right)} \leq c \int_{Q_{1}(0)} d\left(D v_{i}, K\right)+\left|D^{2} v_{i}\right|^{2} .
$$

Approximate principle 2. The minimiser $u$ of $I_{\epsilon}$ is a Lipschitz.

Let us make it once again clear we can not prove either approximate principle, they are simply a device to show the strategy of the proof. Now we split every sub-square $Q_{\frac{1}{M}}\left(c_{i}\right)$ into two right angle triangles, denote them $\tau_{i}, \tau_{i+M^{2}}$ so the set $\left\{\tau_{1}, \tau_{2}, \ldots, \tau_{2 M^{2}}\right\}$ is a triangulation of $\Omega$. Let $\tilde{u}$ be the piecewise affine function we obtain from $u$ by defining $\tilde{u}_{\left\lfloor\tau_{i}\right.}$ to be the affine map we get from interpolating $u$ on the corners of $\tau_{i}$. 
Now for any $i \notin B$ let $\omega_{1}^{i}, \omega_{2}^{i}, \omega_{3}^{i}$ denotes the corners of $\tau_{i}$, so $l, q \in\{1,2,3\}$

$$
\begin{gathered}
\left|D \tilde{u}_{\left\lfloor\tau_{i}\right.}\left(\frac{\omega_{l}^{i}-\omega_{q}^{i}}{\left|\omega_{l}^{i}-\omega_{q}^{i}\right|}\right)-D L_{i}\left(\frac{\omega_{l}^{i}-\omega_{q}^{i}}{\left|\omega_{l}^{i}-\omega_{q}^{i}\right|}\right)\right| \leq M\left|\left(u\left(\omega_{l}^{i}\right)-L_{i}\left(\omega_{l}^{i}\right)\right)-\left(u\left(\omega_{q}^{i}\right)-L_{i}\left(\omega_{q}^{i}\right)\right)\right| \\
\stackrel{(2.1)}{\leq} c \int_{Q_{\frac{1}{M}}\left(c_{i}\right)} M^{2} d(D u, K)+\left|D^{2} u\right|^{2} .
\end{gathered}
$$

Since (2.2) holds true for every $l, q \in\{1,2\}$ we have $\left|D \tilde{u}_{\left\lfloor\tau_{i}\right.}-D L_{i}\right| \leq c \int_{Q_{\frac{1}{M}\left(c_{i}\right)}} M^{2} d(D u, K)+\left|D^{2} u\right|^{2}$. In exactly the same way $\left|D \tilde{u}_{\left\lfloor\tau_{i+M^{2}}\right.}-D L_{i+M^{2}}\right| \leq c \int_{Q_{\frac{1}{M}\left(c_{i}\right)}} M^{2} d(D u, K)+\left|D^{2} u\right|^{2}$. So

$$
\sum_{i \in\left\{1,2, \ldots, M^{2}\right\} \backslash B}\left|D \tilde{u}_{\left\lfloor\tau_{i}\right.}-D L_{i}\right| L^{2}\left(\tau_{i}\right)+\left|D \tilde{u}_{\left\lfloor\tau_{i+M^{2}}\right.}-D L_{i+M^{2}}\right| L^{2}\left(\tau_{i+M^{2}}\right) \leq c m_{\epsilon}^{1}
$$

Now for any $i \in B$, since $u$ is Lipschitz, for $l, q \in\{1,2,3\}$ we have

$$
\left|D \tilde{u}_{\left\lfloor\tau_{i}\right.}\left(\frac{\omega_{l}^{i}-\omega_{q}^{i}}{\left|\omega_{l}^{i}-\omega_{q}^{i}\right|}\right)\right|=\left|\frac{u\left(\omega_{l}^{i}\right)-u\left(\omega_{q}^{i}\right)}{\left|\omega_{l}^{i}-\omega_{q}^{i}\right|}\right| \leq c
$$

thus $d\left(D \tilde{u}_{\left\lfloor\tau_{i}\right.}, K\right) \leq c$ and in the same way $d\left(D \tilde{u}_{\tau_{i+M^{2}}}, K\right) \leq c$ so

$$
\sum_{i \in B}\left|D \tilde{u}_{\left\lfloor\tau_{i}\right.}-D L_{i}\right| L^{2}\left(\tau_{i}\right)+\left|D \tilde{u}_{L \tau_{i+M^{2}}}-D L_{i+M^{2}}\right| L^{2}\left(\tau_{i+M^{2}}\right) \leq \frac{c}{M^{2}} \sum_{i \in B} \int_{Q_{\frac{1}{M}}\left(c_{i}\right)}\left|D^{2} u\right|^{2} \leq c m_{\epsilon}^{1} \cdot(
$$

So as $\left\{\tau_{i}\right\}$ is a $\left(\sqrt{\epsilon}, 10^{-1} \sigma\right)$-triangulation and from (2.3), (2.4) we have $\alpha(\sqrt{\epsilon}) \leq c m_{\epsilon}^{1}$ which establishes the lower bound.

It is easy to construct a counter example to the "morally true" Lemma 1, however as a substitute we have Proposition 5.1, see Section 5. Since $i \in B$ it should seem reasonable that there exists $k_{0}$ such that

$$
\int_{Q_{1}(0)} d\left(D v_{i}, S O(2) A_{k_{0}}\right) \leq c \int_{Q_{1}(0)} d\left(D v_{i}, K\right) .
$$

This follows from a kind of capacity type argument that is Step 1 of Proposition 5.1. Alternatively imagine we had slightly more integrability of $D^{2} v_{i}$ and hence that $\left(\int_{Q_{1}(0)}\left|D^{2} v_{i}\right|^{2+\delta}\right)^{\frac{1}{2+\delta}}$ is "small" (in fact $v_{i}$ satisfies a fourth order elliptic PDE coming from the Euler Lagrange equation of $u$ so we could indeed establish such higher integrability via reverse Holder inequalities), then by Sobolev embedding we would have that $D v_{i}$ stays in a neighbourhood of some well $S O(2) A_{k_{0}}$ and so (2.5) trivially follows.

Now if we were considering the $d^{p}(\cdot, K)$ distance from the wells then we could apply Theorem 3.1 to obtain sharp $L^{p}$ control of the distance of $D v_{i}$ from a matrix in $K$. For the $p=1$ case Theorem 3.1 is false [10] and so we need to use the fact that the "tangent space" to the set $S O(2)$ around the identity is the set of skew symmetric matrices. This allows us to apply the Korn type Poincaré inequality given by Lemma 3.1 to gain sharp control of the $L^{1}$ distance of $v_{i}$ from the affine function.

Note that Proposition 5.1 is not enough since in the argument given in (2.2) we need to control the function exactly at the corners of the triangles. The trick to overcome this is the following. Let $v: Q_{M}(0) \rightarrow \mathbb{R}^{2}$ be defined by $v(z)=u\left(\frac{z}{M}\right) M$. By the Co-area formula we can find a grid of squares of side length 1, labelled 
$S_{1}, S_{2}, \ldots, S_{M^{2}-4 M}$ such that for each $i$ there exists affine function $L_{i}$ with $D L_{i} \in K$ such that

$$
c \int_{\partial S_{i}}\left|v-L_{i}\right|+\left|D^{2} v\right|^{2}+d\left(D v, S O(2) \operatorname{sym}\left(D L_{i}\right)\right) \leq \int_{N_{1}\left(S_{i}\right)} d(D v, K)+\left|D^{2} v\right|^{2}=: \alpha_{i}
$$

(where $\operatorname{sym}(A)$ denotes the symmetric part of matrix $A$ we obtain by polar decomposition). We can split $S_{i}$ into disjoint triangles $\tau_{i}, \tau_{i+M^{2}}$. Let $a_{i}, b_{i}, c_{i}$ be the corners of $\tau_{i}$ where $\left[a_{i}, b_{i}\right] \cup\left[b_{i}, c_{i}\right]=\partial \tau_{i} \cap \partial S_{i}$. The important point is that $D v$ along $\left[a_{i}, b_{i}\right]$ varies by at most $\sqrt{\alpha_{i}}$ and so its not hard to show $D v(z) \in B_{c \sqrt{\alpha_{i}}}\left(D L_{i}\right)$ for all $z \in\left[a_{i}, b_{i}\right]$. For simplicity let us assume $\operatorname{sym}\left(D L_{i}\right)=I d$.

Given $\tilde{b}_{i} \in\left[a_{i}, b_{i}\right]$, by trigonometry this allows to conclude

$$
\left|v\left(a_{i}\right)-v\left(\tilde{b}_{i}\right)\right| \geq\left(1-c \alpha_{i}\right)\left|a_{i}-\tilde{b}_{i}\right| .
$$

And very easily from $(2.6)$ (since we have assumed $\operatorname{sym}\left(D L_{i}\right)=I d$ ) we have

$$
\left|v\left(a_{i}\right)-v\left(\tilde{b}_{i}\right)\right| \leq\left(1+c \alpha_{i}\right)\left|a_{i}-\tilde{b}_{i}\right| .
$$

The point $\tilde{b}_{i}$ can be easily chosen so that $\left|v\left(\tilde{b}_{i}\right)-L_{i}\left(\tilde{b}_{i}\right)\right| \leq c \alpha_{i}$. In exactly the same way we can find $\tilde{c}_{i} \in\left[a_{i}, c_{i}\right]$ such that $\left|v\left(\tilde{c}_{i}\right)-L_{i}\left(\tilde{c}_{i}\right)\right| \leq c \alpha_{i}$ and ||$v\left(a_{i}\right)-v\left(\tilde{c}_{i}\right)|-| a_{i}-\tilde{c}_{i}|| \leq c \alpha_{i}$. Let $\gamma_{1}=\left|a_{i}-\tilde{b}_{i}\right|$ and $\gamma_{2}=\left|a_{i}-\tilde{c}_{i}\right|$ so (defining $\left.N_{\delta}(A):=\{x: d(x, A)<\delta\}\right)$ we have

$$
v\left(a_{i}\right) \in N_{c \alpha_{i}}\left(\partial B_{\gamma_{1}}\left(\tilde{b}_{i}\right)\right) \cap N_{c \alpha_{i}}\left(\partial B_{\gamma_{2}}\left(\tilde{c}_{i}\right)\right) .
$$

See Figure 4. From (2.7) it is not hard to show $v\left(a_{i}\right) \in B_{c \alpha_{i}}\left(L_{i}\left(a_{i}\right)\right)$. We can control the corners $b_{i}, c_{i}$ in the same way. Therefor if we define $l_{i}$ to be the affine map we get from interpolating $v$ on $\left\{a_{i}, b_{i}, c_{i}\right\}$ we have $d\left(D l_{i}, D L_{i}\right) \leq c \alpha_{i}$. Since $\sum_{i} \alpha_{i} \leq c \epsilon^{-1} m_{\epsilon}^{p}$ this gives the lower bound.

\subsection{Upper bound}

To obtain the upper bound we will have to convert a function $v$ that is piecewise affine on a $(\sqrt{\epsilon}, \varsigma)$ triangulation into a function $u \in W^{2,2}(\Omega)$ with affine boundary condition $D u=F$ on $\partial \Omega$ (in the sense of trace), recall we denote the space of such functions by $A_{F}$. The most natural way to do this is to convolve $v$ with a function $\psi_{\sqrt{\epsilon}}$ where $\psi_{\sqrt{\epsilon}}(z):=\epsilon^{-1} \psi\left(\frac{z}{\sqrt{\epsilon}}\right)$ and $\psi \in C_{0}^{\infty}\left(B_{1}(0): \mathbb{R}_{+}\right)$with $\psi=1$ on $B_{\frac{1}{2}}(0)$.

Let $G_{0}:=\left\{i: d\left(D v_{\left\lfloor\tau_{i}\right.}, K\right) \leq \frac{d(S O(2), S O(2) H)}{8}\right\}$ and define $E(x):=\left\{i: \overline{\tau_{i}} \cap B_{\sqrt{\epsilon}}(x) \neq \emptyset\right\}$. Suppose $x \in \Omega$ is such that $E(x) \subset G_{0}$, for simplicity we will assume $d\left(D v_{\left\lfloor\tau_{i}\right.}, S O(2)\right)=d\left(D v_{\left\lfloor\tau_{i}\right.}, K\right)$ for every $i \in E(x)$. Since for any $k, l \in E(x)$ with $H^{1}\left(\bar{\tau}_{k} \cap \bar{\tau}_{l}\right)>0$ we have that there exists $w \in S^{1}$ such that $D v_{\left\lfloor\tau_{k}\right.} w=D v_{\left\lfloor\tau_{l}\right.} w$ and thus $\left|D v_{\left\lfloor\tau_{k}\right.}-D v_{\left\lfloor\tau_{l} \mid\right.}\right| \leq c\left(d\left(D v_{\left\lfloor\tau_{k}\right.}, S O(2)\right)+d\left(D v_{\left\lfloor\tau_{l}\right.}, S O(2)\right)\right)$ because if $D v_{\left\lfloor\tau_{k}\right.} \in S O(2)$ and $D v_{\left\lfloor\tau_{l}\right.} \in S O(2)$ the fact that $D v_{\left\lfloor\tau_{k}\right.} w=D v_{\left\lfloor\tau_{l}\right.} w$ would imply $D v_{\left\lfloor\tau_{k}\right.}=D v_{\left\lfloor\tau_{l}\right.}$, so the difference between $D v_{\left\lfloor\tau_{k}\right.}$ and $D v_{\left\lfloor\tau_{l}\right.}$ is controlled by the distance of these matrices from $S O(2)$.

A relatively easy generalisation of this is that for any $x$ where $E(x) \subset G_{0}$

$$
\left|D v_{\left\lfloor\tau_{k}\right.}-D v_{\left\lfloor\tau_{l} \mid\right.}\right| \leq c \max \left\{d\left(D v_{\left\lfloor\tau_{i}\right.}, K\right): i \in E(x)\right\} \text { for any } k, l \in E(x)
$$


Now $D u(x)=\sum_{i \in E(x)} D v_{\left\lfloor\tau_{i}\right.} \int_{\tau_{i}} \psi_{\sqrt{\epsilon}}(z-x) \mathrm{d} L^{2} z$. Let's pick $i_{0} \in E(x)$ we then have

$$
\begin{aligned}
\left|D u(x)-D v_{\left\lfloor\tau_{i_{0}}\right.}\right| & =\left|\sum_{i \in E(x)}\left(D v_{\left\lfloor\tau_{i}\right.}-D v_{\left\lfloor\tau_{\tau_{0}}\right.}\right) \int_{\tau_{i}} \psi_{\sqrt{\epsilon}}(z-x) \mathrm{d} L^{2} z\right| \\
& \stackrel{(2.8)}{\leq} \quad c \max \left\{d\left(D v_{\left\lfloor\tau_{i}\right.}, K\right): i \in E(x)\right\} .
\end{aligned}
$$

So for any $x \in \Omega$ such that $E(x) \subset G_{0}, d(D u(x), K)$ is comparable to $d\left(D v_{\left\llcorner\tau_{i_{0}}\right.}, K\right)$ with error given by $\max \left\{d\left(D v_{\left\lfloor\tau_{i}\right.}, K\right): i \in E(x)\right\}$ and thus $\int_{\left\{x: E(x) \subset G_{0}\right\}} d^{p}(D u(z), K) \mathrm{d} L^{2} z \leq c \sum_{i} d^{p}\left(D v_{\left\lfloor\tau_{i}\right.}, K\right)$.

Since $|D u(x)| \leq c \sum_{i \in E(x)}\left|D v_{\left\lfloor\tau_{i} \mid\right.}\right|$ thus $d^{p}(D u(x), K) \leq c\left(\sum_{i \in E(x)} d^{p}\left(D v_{\left\lfloor\tau_{i}\right.}, K\right)+1\right)$ so as $L^{2}\left(\left\{x \in \Omega: E(x) \not \subset G_{0}\right\}\right) \leq c L^{2}\left(\bigcup_{i \notin G_{0}} \tau_{i}\right) \leq c m_{\epsilon}^{p}$ we have $\int_{\left\{x: E(x) \not \subset G_{0}\right\}} d^{p}(D u(x), K) \leq c m_{\epsilon}^{p}$.

So all that remains is to control the $\int_{\Omega}\left|D^{2} u\right|^{2}$ term. For $x \in \Omega$ such that $E(x) \subset G_{0}$ this is relatively easy since

$$
D^{2} u(x)=-\int D v(z) \otimes D \psi_{\sqrt{\epsilon}}(z-x) \mathrm{d} L^{2} z
$$

and as $\int D \psi_{\sqrt{\epsilon}}(z-x) \mathrm{d} L^{2} z=0$ we have

$$
\begin{aligned}
D^{2} u(x) & =-\int\left(D v(z)-D v_{\left\lfloor\tau_{i_{0}}\right.}\right) \otimes D \psi_{\sqrt{\epsilon}}(z-x) \mathrm{d} L^{2} z \\
& \leq c \epsilon^{-\frac{1}{2}} \max \left\{\left|D v_{\left\lfloor\tau_{j}\right.}-D v_{\left\lfloor\tau_{i_{0}}\right.}\right|: j \in E(x)\right\} .
\end{aligned}
$$

So

$$
\begin{aligned}
\left|D^{2} u(x)\right|^{2} & \leq c \epsilon^{-1}\left(\max \left\{\left|D v_{\left\lfloor\tau_{j}\right.}-D v_{\left\lfloor\tau_{i_{0}}\right.}\right|: j \in E(x)\right\}\right)^{p} \\
& \stackrel{(2.8)}{\leq} c \epsilon^{-1} \max \left\{d^{p}\left(D v_{\left\lfloor\tau_{i}\right.}, K\right): i \in E(x)\right\} .
\end{aligned}
$$

Thus

$$
\int_{\left\{x: E(x) \subset G_{0}\right\}}\left|D^{2} u(x)\right|^{2} \mathrm{~d} L^{2} x \leq c \epsilon^{-1} \sum_{i} d^{p}\left(D v_{\left\lfloor\tau_{i}\right.}, K\right) L^{2}\left(\tau_{i}\right) \leq c \epsilon^{-1} m_{\epsilon}^{p}
$$

So far everything goes well simply by using (2.8), however for $x \in \Omega$ such that $E(x) \not \subset G_{0}$ we have a problem because the quantity we are interested in is $\left|D^{2} u(x)\right|^{2}$ and from equation (2.10), if the jump from $D v_{\left\lfloor\tau_{i}\right.}$ to $D v_{\left\lfloor\tau_{l}\right.}$ is much greater than 1 we can not estimate $\left|D^{2} u\right|^{2}$ by any $L^{1}$ control of the distance of $D v$ from $K$. Quite simply if we have an arbitrary function $v \in \mathcal{F}_{F}^{(\varsigma, \sqrt{\epsilon})}$ and we form function $u$ by convolving it with $\psi_{\sqrt{\epsilon}}$ it could be the case that $\int_{\Omega} d^{p}(D u, K)+\left|D^{2} u\right|^{2} \gg m_{\epsilon}^{p}$. In order for the estimate we want to hold true we need some condition that bounds the square of all the jumps of order $>1$ by the quantity $\epsilon^{-1} m_{\epsilon}^{p}$. The way we deal with this problem is by circumventing it: in establishing the lower bound we showed that from a function $u \in A_{F}$ we can create a function $\tilde{u}$ that is piecewise affine on a $(\sqrt{\epsilon}, \varsigma)$ triangulation and $\int_{\Omega} d(D \tilde{u}, K) \leq \mathrm{cm}_{\epsilon}^{p}$, if we were smarter we could show the function $\tilde{u}$ that we created had even stronger properties. For example if $u$ was Lipschitz then $\tilde{u}$ would also be Lipschitz and our problems would be over. Unfortunately we can not prove $u$ is Lipschitz, however what we have for free is that $\int_{\Omega}\left|D^{2} u\right|^{2} \leq \epsilon^{-1} m_{\epsilon}^{p}$. It turns out that for sufficiently careful choice of triangulation this is strong enough for us to be able to construct a function $\tilde{u}$ such that if we define 
$N_{i}(\tilde{u}), J(\tilde{u})$ by $(1.3),(1.4)$ we have that

$$
\sum_{i \in J(\tilde{u}))} \sum_{M \in N_{i}(\tilde{u})}\left|D \tilde{u}_{\left\lfloor\tau_{i}\right.}-M\right|^{2} \leq c \epsilon^{-1} m_{\epsilon}^{p} .
$$

So we define a function space we call $\mathcal{D}_{F}^{(\varsigma, h)}$ to be the set of piecewise affine functions in $\mathcal{F}_{F}^{(\varsigma, h)}$ that satisfies (2.11) and we will show in the "lower bound" part of Theorem 1.1 that given $u \in A_{F}$ with $I_{\epsilon}^{p}(u) \leq c m_{\epsilon}^{p}$ we can construct function $\tilde{u} \in \mathcal{D}_{F}^{(\varsigma, \sqrt{\epsilon})}$ from it such that $\int_{\Omega} d^{p}(D \tilde{u}, K) \leq c m_{\epsilon}^{p}$.

To prove the "upper bound" we will need to show that if $v \in \mathcal{D}_{F}^{(\varsigma, \sqrt{\epsilon})}$ then we can construct function $u \in A_{F}$ and $I_{\epsilon}^{p}(u) \leq c \int_{\Omega} d^{p}(D v, K)$. It turns out that proceeding in the "naive" way and simply defining $u=v * \psi_{\sqrt{\epsilon}}$ inequality (2.11) is strong enough to conclude $\int_{\Omega}\left|D^{2} u\right|^{2} \leq \epsilon^{-1} m_{\epsilon}^{p}$, in some sense from equation (2.10) this should come as no great surprise. Since we have already shown $\int_{\Omega} d^{p}(D u, K) \leq m_{\epsilon}^{p}$ the upper bound is completed.

\section{BACKGROUND}

We will need a couple of not so well known Poincaré inequalities. Firstly a Korn type Poincaré inequality from [21], for a form more convenient for our purposes we refer to Theorem 6.5 [1]. The lemma we state is highly simplified version of Theorem 6.5 .

Lemma 3.1. Let $u \in W^{1,1}\left(\Omega: \mathbb{R}^{m}\right)$ we have a constant $c_{0}=c_{0}(n)$ such that for any $B_{r}(x) \subset \Omega$ there exists vectors $a_{x, r} \in \mathbb{R}^{m}$ and matrix $b_{x, r} \in M^{m \times n}$

$$
\int_{B_{r}(x)}\left|u(z)-b_{x, r} \cdot(z-x)-a_{x, r}\right| \mathrm{d} L^{n} z \leq c_{0} r \int_{B_{r}(x)}\left|\frac{D u(z)+D u^{T}(z)}{2}\right| \mathrm{d} L^{n} z .
$$

Secondly a version of the more standard Poincaré inequality.

Lemma 3.2. Let $a_{0}>0$ be a fixed small constant. Let $p \geq 1$. Suppose $u \in W^{1, p}\left(B_{1}(0)\right)$ is such that $L^{n}(\{x: u(x)=0\})>a_{0}$. There exists constant $c_{1}=c_{1}\left(a_{0}, n\right)$

$$
\int_{B_{1}(0)}|u(z)|^{p} \mathrm{~d} L^{n} z \leq c_{1} \int_{B_{1}(0)}|D u(z)|^{p} \mathrm{~d} L^{n} z
$$

Proof of Lemma 3.2. Since this lemma is essentially standard we only sketch its proof. Suppose (3.1) is false, then we have a sequence $u_{n} \in W^{1, p}\left(B_{1}(0)\right)$ such that

$$
\left(\int_{B_{1}(0)}\left|u_{n}(z)\right|^{p} \mathrm{~d} L^{n} z\right)\left(\int_{B_{1}(0)}\left|D u_{n}(z)\right|^{p} \mathrm{~d} L^{n} z\right)^{-1} \rightarrow \infty .
$$

Let $w_{n}(x):=u_{n}(x)\left(\int_{B_{1}(0)}\left|u_{n}(z)\right|^{p} \mathrm{~d} L^{n} z\right)^{-1}$. So $\left\|w_{n}\right\|_{L^{p}\left(B_{1}(0)\right)}=1$ and $\left\|D w_{n}\right\|_{L^{p}\left(B_{1}(0)\right)} \stackrel{(21)}{\rightarrow} 0$ as $n \rightarrow \infty$. By BV compactness theorem (see Thm. 3.22 [2]) there exists a subsequence of $w_{n}$ that has a limit $w \in B V\left(B_{1}(0)\right)$ where $|D w|\left(B_{1}(0)\right)=0$ and $\int_{B_{1}(0)} w=1$ with $L^{2}(\{x: w(x)=0\}) \geq a_{0}$, which is a contradiction.

A theorem that we will use many times is the following [18].

Theorem 3.1 (Friesecke, James, Müller). Let $U$ be a bounded Lipschitz domain in $\mathbb{R}^{n}, n \geq 2$. Let $q>1$. There exists a constant $C(U, q)$ with the following property. For each $v \in W^{1, q}\left(U, \mathbb{R}^{n}\right)$ there exists an associated rotation $R \in S O(n)$ such that

$$
\|D v-R\|_{L^{q}(U)} \leq C(U, q)\|\operatorname{dist}(D v, S O(n))\|_{L^{q}(U)} .
$$




\section{Rough LOWER BOUNDS ON $m_{\epsilon}^{p}$}

Lemma 4.1. Let $p \geq 1$, define

$$
m_{\epsilon}^{p}:=\inf _{u \in A_{F}} \int_{\Omega} d^{p}(D u(z), K)+\epsilon\left|D^{2} u(z)\right|^{2} \mathrm{~d} L^{2} z .
$$

We have positive constant $c_{1}$ (depending only on $\sigma, p$ ) such that

$$
m_{\epsilon}^{p} \geq c_{1} \epsilon^{\frac{1}{2}} \text { for all } \epsilon>0 .
$$

Proof. Let

$$
d_{0}:=\frac{1}{4} \inf \left\{|A-B|: A \in S O(2) A_{i}, B \in S O(2) A_{j}, i \neq j\right\} .
$$

By density of smooth functions in $W^{2,2}(\Omega)$ we can find a smooth function $u$ satisfying $u(x)=l_{F}(x)$ for $x \in \partial \Omega$ with

$$
\int_{\Omega} d^{p}(D u(x), K)+\epsilon\left|D^{2} u(x)\right|^{2} \mathrm{~d} L^{2} x \leq \max \left\{2 m_{\epsilon}^{p}, c_{1} \epsilon^{\frac{1}{2}}\right\} .
$$

Now suppose (4.2) is false, so for some small positive constant $c_{1}<d_{0}$ we have $m_{\epsilon}^{p} \leq c_{1} \epsilon^{\frac{1}{2}}$. By Cauchy Schwartz inequality we have

$$
\int_{\Omega} d^{\frac{p}{2}}(D u(x), K)\left|D^{2} u(x)\right| \mathrm{d} L^{2} x \leq 2 c_{1} .
$$

Let $U_{i}:=\left\{x \in \Omega: d\left(D u(x), S O(2) A_{i}\right)<c_{1}\right\}$. There must exists $i_{0} \in\{1,2, \ldots, N\}$ such that $L^{2}\left(U_{i_{0}}\right) \geq$ $\frac{L^{2}(\Omega)-c \epsilon^{\frac{1}{2 p}}}{N}$. Let $E(x)=d^{\frac{p}{2}}(D u(x), K)\left|D^{2} u(x)\right|$ and $\psi_{z}: \mathbb{R}^{2} \rightarrow[0,2 \pi)$ be defined by $|x-z| \mathrm{e}^{i \psi_{z}(x)}=x-z$. Note $\psi_{z}$ is smooth in $\mathbb{R}^{2} \backslash\left\{\left(z_{1}, z_{2}+\lambda\right): \lambda \in \mathbb{R}_{+}\right\}=: \mathbb{U}_{z}$ and $\left|D \psi_{z}(x)\right| \leq \frac{1}{|x-z|}$ for any $x \in \mathbb{U}_{z}$. Let $c_{0}:=$ $\sup \left\{\int_{\Omega} \frac{1}{|z-x|} \mathrm{d} L^{2} z: x \in \Omega\right\}$. We know via Fubini theorem

$$
\begin{aligned}
\int_{\Omega} \int_{\Omega} E(x)\left|D \psi_{z}(x)\right| \mathrm{d} L^{2} x \mathrm{~d} L^{2} z & \leq \int_{\Omega} E(x)\left(\int_{\Omega} \frac{1}{|z-x|} \mathrm{d} L^{2} z\right) \mathrm{d} L^{2} x \\
& \leq c_{0} \int_{\Omega} E(x) \mathrm{d} L^{2} x \\
& \stackrel{(4.5)}{\leq} 2 c_{0} c_{1} .
\end{aligned}
$$

So we can find a subset $G \subset \Omega$ such that $L^{2}(\Omega \backslash G) \leq 2 c_{0} c_{1}^{\frac{1}{3}}$ and for every $z \in G$ we have

$$
\int_{\Omega} E(x)\left|D \psi_{z}(x)\right| \mathrm{d} L^{2} x \leq c_{1}^{\frac{2}{3}} .
$$

Now by the Co-area formula, for each $z \in G$ we can find $\Psi_{z} \subset[0,2 \pi)$ with $L^{1}\left([0,2 \pi) \backslash \Psi_{z}\right) \leq c_{1}^{\frac{1}{3}}$ for every $\theta \in \Psi_{z}$ we have $\int_{\left(z+\left\langle\mathrm{e}^{\mathrm{i} \theta}\right\rangle\right) \cap \Omega} E(x) \mathrm{d} H^{1} x \leq c_{1}^{\frac{1}{3}}$. We can assume $c_{1}$ is sufficiently small so $G \cap U_{i_{0}} \neq \emptyset$. Now we claim for each $z \in G \cap U_{i_{0}}$ we have that

$$
\sup \left\{d\left(D u(x), S O(2) A_{i_{0}}\right): x \in\left(\bigcup_{\theta \in \Psi_{z}}\left(z+\left\langle\mathrm{e}^{\mathrm{i} \theta}\right\rangle\right)\right) \cap \Omega\right\} \leq 4 c_{1}^{\frac{2}{3(2+p)}} .
$$


Suppose (4.6) is false. So there exists $z_{0} \in G \cap U_{i_{0}}$ and $\theta_{0} \in \Psi_{z_{0}}$ with $z_{1} \in\left(z_{0}+\left\langle\mathrm{e}^{\mathrm{i} \theta}\right\rangle\right) \cap \Omega$ such that $d\left(D u\left(z_{1}\right), S O(2) A_{i_{0}}\right)>4 c_{1}^{\frac{2}{3(2+p)}}$. So as $d\left(D u\left(z_{0}\right), S O(2) A_{i_{0}}\right)<c_{1}$ we can find $z_{2}, z_{3} \in\left[z_{0}, z_{1}\right]$ with the properties

$$
d\left(D u\left(z_{2}\right), S O(2) A_{i_{0}}\right)=c_{1}^{\frac{2}{3(2+p)}} \text { and } d\left(D u\left(z_{3}\right), S O(2) A_{i_{0}}\right)=4 c_{1}^{\frac{2}{3(2+p)}} .
$$

In addition we have

$$
d\left(D u(z), S O(2) A_{i_{0}}\right) \in\left[c_{1}^{\frac{2}{3(2+p)}}, 4 c_{1}^{\frac{2}{3(2+p)}}\right] \text { for any } z \in\left[z_{2}, z_{3}\right]
$$

So $c_{1}^{\frac{1}{3}} \geq \int_{z_{2}}^{z_{3}} E(z) \mathrm{d} H^{1} z \geq c_{1}^{\frac{p}{3(2+p)}} \int_{z_{2}}^{z_{3}}\left|D^{2} u(z)\right| \mathrm{d} H^{1} z \geq 3 c_{1}^{\frac{1}{3}}$ which is a contradiction. So pick $z_{0} \in G \cap U_{i_{0}}$ and let $\Lambda=\left(\bigcup_{\theta \in \Psi_{z_{0}}}\left(z_{0}+\left\langle\mathrm{e}^{i \theta}\right\rangle\right)\right) \cap \Omega$. Note that

$$
\begin{aligned}
L^{2}(\Omega \backslash \Lambda) & \leq L^{2}\left(\left(\bigcup_{\theta \in[0,2 \pi) \backslash \Psi_{z_{0}}}\left(z_{0}+\left\langle\mathrm{e}^{\mathrm{i} \theta}\right\rangle\right)\right) \cap B_{\operatorname{diam}(\Omega)}(0)\right) \\
& \leq 2 \pi \operatorname{diam}(\Omega) c_{1}^{\frac{1}{3}} .
\end{aligned}
$$

So as for any $x \in \Omega \backslash \Lambda$ we have $d\left(D u(x), S O(2) A_{i_{0}}\right) \leq d(D u(x), K)+c$ thus

$$
\begin{aligned}
& \int_{\Omega} d\left(D u(x), S O(2) A_{i_{0}}\right) \mathrm{d} L^{2} x \leq \int_{\Omega} d(D u(x), K) \mathrm{d} L^{2} x+c L^{2}(\Omega \backslash \Lambda) \\
& \stackrel{(4.8)}{\leq} 2 \pi \operatorname{diam}(\Omega) c_{1}^{\frac{1}{3}}+c \epsilon^{\frac{1}{2 p}} .
\end{aligned}
$$

So applying Proposition 2.6 [9] we have that there exists $R_{0} \in S O(2)$ such that

$$
\int_{\Omega}\left|D u(x)-R_{0} A_{i_{0}}\right| \mathrm{d} L^{2} x \leq c c_{1}^{\frac{1}{6}} .
$$

Since $R_{0} A_{i_{0}} \neq F$ there must exist $w \in S^{1}$ such that $R_{0} A_{i_{0}} w \neq F w$. We must be able to find $m \in w^{\perp} \cap$ $B_{\frac{\text { diam }(\Omega)}{10}}(0)$ such that $\int_{\Omega \cap(m+\langle w\rangle)}\left|D u(z)-R_{0} A_{i_{0}}\right| \mathrm{d} L^{1} z \leq c c_{1}^{\frac{1}{12}}$. Let $a, b$ denote the endpoints of $\Omega \cap(c+\langle m\rangle)$. We have

$$
\left|F(a-b)-R_{0} A_{i_{0}}(a-b)\right| \leq\left|\int_{a}^{b}\left(D u(z)-R_{0} A_{i_{0}}\right) w \mathrm{~d} L^{1} z\right| \leq c c_{1}^{\frac{1}{12}}
$$

which is a contradiction assuming $c_{1}$ is chosen small enough.

\section{Proof of Theorem 1.1}

Proposition 5.1. Let $K=S O(2) A_{1} \cup \ldots S O(2) A_{N}$, let $\sigma$ be the minimum of the absolute values of the eigenvalues of $A_{i}$. Let $p \geq 1$, suppose $u \in W^{2,2}\left(B_{1}(0): \mathbb{R}^{2}\right)$ satisfies the following properties

$$
\begin{gathered}
\int_{B_{1}(0)} d^{p}(D u(z), K) \mathrm{d} L^{2} z \leq \beta \\
\int_{B_{1}(0)}\left|D^{2} u(z)\right|^{2} \mathrm{~d} L^{2} z \leq \beta
\end{gathered}
$$


then in the case $p>1$ there exists matrix $M \in K$ such that

$$
\int_{B_{1}(0)}|D u(z)-M|^{p} \mathrm{~d} L^{2} z \leq c \beta .
$$

And for the case $p=1$ there exists $i_{0} \in\{1,2, \ldots, N\}$ and affine function $L: B_{1}(0) \rightarrow \mathbb{R}^{2}$ with $D L \in S O(2) A_{i_{0}}$ such that

$$
\int_{B_{\sigma^{2}}(0)}|u(z)-L(z)| \mathrm{d} L^{2} z \leq c \beta
$$

and

$$
\int_{B_{1}(0)} d\left(D u(z), S O(2) A_{i_{0}}\right) \mathrm{d} L^{2} z \leq c \beta .
$$

Proof.

Step 1. Recall definition (4.3) of $d_{0}$, let $d_{1}=\frac{\sigma}{10} d_{0}$ and let

$$
U_{i}:=\left\{x \in B_{1}(0): d\left(D u(x), S O(2) A_{i}\right)<d_{1}\right\} \text { for } i=1,2, \ldots, N .
$$

We will show there exists $i_{0} \in\{1,2, \ldots, N\}$ such that

$$
L^{2}\left(B_{1}(0) \backslash U_{i_{0}}\right) \leq c \beta .
$$

As a consequence we will establish (5.5).

Proof of Step 1 . Since for any $x \in B_{1}(0) \backslash\left(\bigcup_{i=1}^{N} U_{i}\right)$ we have $d(D u(x), K)>d_{1}$. So

$$
L^{2}\left(B_{1}(0) \backslash\left(\bigcup_{i=1}^{N} U_{i}\right)\right) \leq \frac{1}{d_{1}^{p}} \int_{B_{1}(0)} d^{p}(D u(z), K) \mathrm{d} L^{2} z \stackrel{(5.1)}{\leq} c \beta
$$

which implies there must exists $i_{0} \in\{1,2, \ldots, N\}$ such that $L^{2}\left(U_{i_{0}}\right) \geq \frac{c}{N}$.

Let $\gamma \in\left(0, \frac{d_{1}}{4}\right)$ be some very small number. We define

$$
\mathcal{S}(z):= \begin{cases}z-3 \gamma & \text { for } z>3 \gamma \\ 0 & \text { for } z \leq 3 \gamma\end{cases}
$$

and $\mathcal{T}(z)=\int \mathcal{S}(x) \psi_{\gamma}(z-x) \mathrm{d} L^{1} z$ where $\psi_{\gamma}(z):=\psi\left(\frac{z}{\gamma}\right) \gamma^{-1}$ and $\psi$ is the standard one dimensional convolution kernel with $\int \psi=1$ and $\psi \equiv 1$ on $\left[-\frac{1}{4}, \frac{1}{4}\right]$. Let $\mathcal{P}_{0}: M^{2 \times 2} \rightarrow \mathbb{R}$ be defined by $\mathcal{P}_{0}(M)=$ $\mathcal{T}\left(d\left(M, S O(2) A_{i_{0}}\right)-d_{1}\right)$ note that $\mathcal{P}_{0}$ is smooth and Lipschitz. We define $f(z):=\mathcal{P}_{0}(D u(z))$ it is easy to see that $f \in W^{1,2}\left(B_{1}(0)\right)$ and we have $|D f(z)| \leq c\left|D^{2} u(z)\right|$, hence $\int_{B_{1}(0)}|D f(z)|^{2} \mathrm{~d} L^{2} z \leq c \beta$. We also know we have $f(z)=0$ for any $z \in U_{i_{0}}$ and so by Lemma 3.2 we have that $\int_{B_{1}(0)}|f(z)|^{2} \mathrm{~d} L^{2} z \leq c \beta$. As $f(z) \geq d_{1}$ for any $z \in \bigcup_{i \in\{1,2, \ldots, N\} \backslash\left\{i_{0}\right\}} U_{i}$ together with (5.8) this implies (5.7).

Note $(d(D u(z), K)+c)^{p} \leq d^{p}(D u(z), K)+c$

$$
\begin{gathered}
\int_{B_{1}(0)} d^{p}\left(D u(z), S O(2) A_{i_{0}}\right) \mathrm{d} L^{2} z \\
\quad \leq \int_{B_{1}(0)} d^{p}(D u(z), K) \mathrm{d} L^{2} z+c L^{2}\left(B_{1}(0) \backslash U_{i_{0}}\right) \\
\stackrel{(5.1),(5.7)}{\leq} c \beta .
\end{gathered}
$$


Now for $p>1$ by Theorem 3.1 there exists $R_{0} \in S O(2)$ such that $\int_{B_{1}(0)}\left|D u(z)-R_{0} A_{i_{0}}\right|^{p} \mathrm{~d} L^{2} z \leq c \beta$ which establishes (5.3). Obviously inequality (5.9) also gives (5.5) for $p=1$.

Step 2. Let $P_{0}$ be the affine function with $P_{0}(0)=0, D P_{0}=A_{i_{0}}^{-1}$. Define $v: B_{\sigma}(0) \rightarrow \mathbb{R}^{2}$ by $v(z)=u\left(P_{0}(z)\right)$. We will show there exists and affine function $L_{1}$ such that

$$
\int_{B_{\sigma}(0)}\left|v(z)-L_{1}(z)\right| \mathrm{d} L^{2} z \leq c \beta .
$$

Proof of Step 2. Firstly we apply the truncation theorem Proposition A.1. [18]. So there exists a Lipschitz function $\tilde{v}$ with $\|D \tilde{v}\|_{L^{\infty}\left(B_{\sigma}(0)\right)} \leq C$ and

$$
L^{2}\left(\left\{x \in B_{\sigma}(0): \tilde{v}(x) \neq v(x)\right\}\right) \leq c \int_{\left\{x \in B_{\sigma}(0):|D v(x)|>C\right\}}|D v(z)| \mathrm{d} L^{2} z \leq c \beta
$$

and

$$
\|D v-D \tilde{v}\|_{L^{1}\left(B_{\sigma}(0)\right)} \leq c \int_{\left\{x \in B_{\sigma}(0):|D v(x)|>C\right\}}|D v(z)| \mathrm{d} L^{2} z \leq c \beta
$$

Note

$$
\begin{gathered}
\int_{B_{\sigma}(0)} d(D \tilde{v}(z), S O(2)) \mathrm{d} L^{2} z \stackrel{(5.12)}{\stackrel{(5.9)}{\leq}} \int_{B_{\sigma}(0)} d(D v(z), S O(2)) \mathrm{d} L^{2} z+c \beta \\
\leq \quad c .
\end{gathered}
$$

Thus by Theorem 3.1 we have that there exists $R_{0}$ such that

$$
\begin{gathered}
\int_{B_{\sigma}(0)}\left|D \tilde{v}(x)-R_{0}\right|^{2} \mathrm{~d} L^{2} x \leq c \int_{B_{\sigma}(0)} d^{2}(D \tilde{v}(x), S O(2)) \mathrm{d} L^{2} x \\
\stackrel{(5.13)}{\leq} c \beta .
\end{gathered}
$$

Let $l_{R_{0}}$ be an affine function with $D l_{R_{0}}=R_{0}$ and $l_{R_{0}}(0)=0$, we define $w(x)=\tilde{v}\left(l_{R_{0}}(x)\right)$. So from (5.14) we have

$$
\int_{B_{\sigma}(0)}|D w(x)-I d|^{2} \mathrm{~d} L^{2} x \leq c \beta .
$$

Now linearising $d(\cdot, S O(2))$ near the identity we have

$$
\begin{aligned}
d(G, S O(2)) & =\left|\frac{1}{2}\left(G+G^{T}\right)-I d\right|+O\left(|G-I d|^{2}\right) \\
& =|\operatorname{sym}(G-I d)|+O\left(|G-I d|^{2}\right) .
\end{aligned}
$$

So we have

$$
\begin{aligned}
\int_{B_{\sigma}(0)}|\operatorname{sym}(D w(x)-I d)| \mathrm{d} L^{2} x \leq & c \int_{B_{\sigma}(0)}|D w(x)-I d|^{2} \mathrm{~d} L^{2} x \\
& +c \int_{B_{\sigma}(0)} d(D w(x), S O(2)) \mathrm{d} L^{2} x \\
& \stackrel{(5.15),(5.13)}{\leq} \quad c \beta .
\end{aligned}
$$


Now by Lemma 3.1 we have that there exists an affine function $L_{0}: B_{\sigma}(0) \rightarrow \mathbb{R}^{2}$ such that

$$
\int_{B_{\sigma}(0)}\left|w(x)-x-L_{0}(x)\right| \mathrm{d} L^{2} x \leq c \beta
$$

which gives us an affine function $L_{1}: B_{\sigma}(0) \rightarrow \mathbb{R}^{2}$ with the property that

$$
\int_{B_{\sigma}(0)}\left|\tilde{v}(x)-L_{1}(x)\right| \mathrm{d} L^{2} x \leq c \beta .
$$

Now note by Lemma 3.2 we know that

$$
\int_{B_{\sigma}(0)}|\tilde{v}(x)-v(x)| \mathrm{d} L^{2} x \leq \int_{B_{\sigma}(0)}|D \tilde{v}(x)-D v(x)| \mathrm{d} L^{2} x \stackrel{(5.12)}{\leq} c \beta .
$$

Thus

$$
\int_{B_{\sigma}(0)}\left|v(x)-L_{1}(x)\right| \mathrm{d} L^{2} x \stackrel{(5.17),(5.18)}{\leq} c \beta .
$$

Step 3. We will show there exists $R_{0} \in S O(2)$ such that

$$
\left|D L_{1}-R_{0}\right| \leq c \beta
$$

Proof of Step 3. It is immediate from (5.2) that $\int_{B_{\sigma}(0)}\left|D^{2} v(x)\right|^{2} \mathrm{~d} L^{2} x \leq c \beta$. And so by Holder $\int_{B_{\sigma}(0)}\left|D^{2} v(x)\right| \mathrm{d} L^{2} x \leq c \sqrt{\beta}$. We also know that

$$
\int_{B_{\sigma}(0)} d(D v(x), S O(2)) \mathrm{d} L^{2} x \stackrel{(5.9)}{\leq} c \beta .
$$

Let $\mathcal{C}_{3}$ be some large positive number we decide on later

$$
H_{0}:=\left\{x \in B_{\sigma}(0):\left|L_{1}(z)-v(z)\right| \leq \mathcal{C}_{3} \beta\right\}
$$

Assuming constant $\mathcal{C}_{3}$ is large enough we have from (5.10) that

$$
L^{2}\left(B_{\sigma}(0) \backslash H_{0}\right) \leq \frac{\sigma^{2}}{1000} .
$$

Let $w \in S^{1}$. We define

$$
G_{w}^{1}:=\left\{y \in P_{w^{\perp}}\left(B_{\frac{\sigma}{2}}(0)\right): \int_{P_{w^{\perp}}^{-1}(y) \cap B_{\frac{\sigma}{2}}(0)} d(D v(z), S O(2)) \mathrm{d} H^{1} z \leq \mathcal{C}_{3} \beta\right\}
$$

and

Assuming $\mathcal{C}_{3}$ was chosen large enough we have that

$$
G_{w}^{2}:=\left\{y \in P_{w^{\perp}}\left(B_{\frac{\sigma}{2}}(0)\right): \int_{P_{w \perp}^{-1}(y) \cap B_{\frac{\sigma}{2}}(0)}\left|D^{2} v(z)\right|^{2} \mathrm{~d} H^{1} z \leq \mathcal{C}_{3} \beta\right\} .
$$

$$
L^{1}\left(P_{w^{\perp}}\left(B_{\frac{\sigma}{2}}(0)\right) \backslash G_{w}^{1}\right) \leq \frac{\sigma^{2}}{1000} \text { and } L^{1}\left(P_{w^{\perp}}\left(B_{\frac{\sigma}{2}}(0)\right) \backslash G_{w}^{2}\right) \leq \frac{\sigma^{2}}{1000} .
$$


Now by (5.22) we can pick $y \in G_{w}^{1} \cap G_{w}^{2}$ such that

$$
L^{1}\left(P_{w^{\perp}}^{-1}(y) \cap B_{\frac{\sigma}{2}}(0) \cap H_{0}\right)>\frac{\sigma}{100} .
$$

So we can pick $a, b \in P_{w^{\perp}}^{-1}(y) \cap B_{\frac{\sigma}{2}}(0) \cap H_{0}$ such that $|a-b|>\frac{\sigma}{100}$. We have that

$$
\int_{[a, b]} d(D v(z), S O(2)) \mathrm{d} H^{1} z \leq c \beta
$$

and

$$
\int_{[a, b]}\left|D^{2} v(z)\right| \mathrm{d} H^{1} z \leq c \sqrt{\beta}
$$

For each $z \in[a, b]$ let $R(z) \in S O(2)$ be such that $d(D v(z), S O(2))=|D v(z)-R(z)|$. From (5.23) and (5.24) we have that there exists $R_{0} \in S O(2)$ such that

$$
\sup \left\{\left|D v(z)-R_{0}\right|: z \in[a, b]\right\} \leq c \sqrt{\beta}
$$

Now note

$$
\begin{aligned}
(v(a)-v(b)) \cdot R_{0} v_{1} & =\left(\int_{[a, b]} D v(z) v_{1} \mathrm{~d} H^{1} z\right) \cdot R_{0} v_{1} \\
& \stackrel{(5.23)}{\geq} \int_{[a, b]} R(z) e_{1} \cdot R_{0} e_{1} \mathrm{~d} H^{1} z-c \beta .
\end{aligned}
$$

By definition of $R(z)$, we have that $|D v(z)-R(z)| \leq\left|D v(z)-R_{0}\right| \stackrel{(5.25)}{\leq} c \sqrt{\beta}$. So

$$
\left|R(z)-R_{0}\right| \leq\left|D v(z)-R_{0}\right|+|D v(z)-R(z)| \stackrel{(5.25)}{\leq} c \sqrt{\beta}
$$

Let $\psi \in[0,2 \pi)$ be such that $R_{0}=\left(\begin{array}{cc}\sin \psi & \cos \psi \\ -\cos \psi & \sin \psi\end{array}\right)$ and $\psi(z) \in[0,2 \pi)$ be such that $R(z)=\left(\begin{array}{cc}\sin \psi(z) & \cos \psi(z) \\ -\cos \psi(z) & \sin \psi(z)\end{array}\right)$. We know $\sup \{|\psi-\psi(z)|: z \in[a, b]\} \leq c \sqrt{\beta}$ so

$$
\begin{aligned}
\int_{[a, b]} R(z) e_{1} \cdot R_{0} e_{1} \mathrm{~d} H^{1} z & =\int_{[a, b]} \cos (\psi(z)-\psi) \mathrm{d} H^{1} z \\
& \geq|a-b|-c \beta .
\end{aligned}
$$

Putting this together with (5.26) we have $(v(a)-v(b)) \cdot R_{0} v_{1} \geq|a-b|-c \beta$ which of course implies

$$
|v(a)-v(b)| \geq|a-b|-c \beta
$$

Now

$$
\begin{aligned}
|v(a)-v(b)| & \leq \int_{[a, b]}\left|D v(z) \frac{a-b}{|a-b|}\right| \mathrm{d} H^{1} z \\
& \leq|a-b|+c \beta .
\end{aligned}
$$


Since $a, b \in H_{0}$ we have

$$
|| L_{1}(a-b)|-| a-b|| \stackrel{(5.21)}{\leq}|| v(a)-v(b)|-| a-b||+c \beta \stackrel{(5.27),(5.28)}{\leq} c \beta
$$

which gives

$$
|| L_{1}(w)|-1| \leq c \beta \text { for all } w \in S^{1} .
$$

Let us take three points $x_{1}, x_{2}, x_{3}$ that form the corners of an equilateral triangle, i.e. $\left|x_{i}-x_{j}\right|=1$ for $i, j \in\{1,2,3\}$. So $L_{1}\left(x_{1}\right), L_{1}\left(x_{2}\right), L_{1}\left(x_{3}\right)$ form the corners of a triangle which we denote by $T_{1}$.

Let $\theta_{i}$ denote the angle of the triangle $T_{1}$ at the corner $L_{1}\left(x_{i}\right)$. Let $A_{1}=\left|L_{1}\left(x_{2}\right)-L_{1}\left(x_{3}\right)\right|, A_{2}=$ $\left|L_{1}\left(x_{1}\right)-L_{1}\left(x_{3}\right)\right|, A_{3}=\left|L_{1}\left(x_{1}\right)-L_{1}\left(x_{2}\right)\right|$. Now by the law of $\operatorname{sins} \frac{\sin \theta_{1}}{A_{1}}=\frac{\sin \theta_{2}}{A_{2}}=\frac{\sin \theta_{3}}{A_{3}}$. Let $i, j \in\{1,2,3\}$, $\frac{\sin \theta_{i}}{A_{i}}=\frac{\sin \theta_{j}}{A_{j}}=\frac{\sin \theta_{j}}{A_{i}}+\sin \theta_{j}\left(\frac{1}{A_{j}}-\frac{1}{A_{i}}\right)$. So $\frac{\sin \theta_{i}-\sin \theta_{j}}{A_{i}}=\sin \theta_{j}\left(\frac{A_{i}-A_{j}}{A_{j} A_{i}}\right)$. Note $A_{1}=\left|L_{1}\left(x_{1}-x_{3}\right)\right| \stackrel{(5.29)}{\epsilon}$ $(1-c \beta, 1+c \beta)$. In the same way $1-c \beta \leq A_{i} \leq 1+c \beta$ for $i=2,3$ so

$$
\left|\sin \theta_{i}-\sin \theta_{j}\right| \leq c\left|A_{i}-A_{j}\right|<c \beta .
$$

Now assuming $\beta$ is small enough we must have $\theta_{i} \in\left(0, \frac{999 \pi}{2000}\right)$ for $i=1,2,3$ since otherwise $\max \left\{\left|L_{1}\left(x_{i}\right)-L_{1}\left(x_{j}\right)\right|: i, j \in\{1,2,3\}, i \neq j\right\}>\sqrt{2}-\frac{1}{50}$ which contradicts (5.29). So

$$
\left|\theta_{i}-\theta_{j}\right| \leq c\left|\sin \theta_{i}-\sin \theta_{j}\right| \stackrel{(5.29)}{\leq} c \beta
$$

Since $\theta_{1}+\theta_{2}+\theta_{3}=\pi$ this gives $\left|\theta_{i}-\frac{\pi}{3}\right| \leq c \beta$ for $i=1,2,3$ which implies there exists rotation $R_{0} \in S O(2)$ such that $\left|D L_{1}-R_{0}\right| \leq c \beta$ which completes the proof of Step 3.

Proof of Proposition 5.1 completed. Let $L_{0}$ be the affine function with $L_{0}(0)=L_{1}(0)$ and $D L_{0}=R_{0}$ where $R_{0} \in S O(2)$ satisfies (5.19) of Step 3. So from (5.10) we know

$$
\int_{B_{\sigma}(0)}\left|v(x)-L_{0}(x)\right| \mathrm{d} L^{2} x \leq c \beta .
$$

As $u(z)=v\left(P_{0}^{-1}(z)\right)$ we have that

$$
\int_{B_{\sigma^{2}}(0)}\left|u(z)-L_{0}\left(P_{0}^{-1}(z)\right)\right| \mathrm{d} L^{2} z=\int_{B_{\sigma^{2}}(0)}\left|v\left(P_{0}^{-1}(z)\right)-L_{0}\left(P_{0}^{-1}(z)\right)\right| \mathrm{d} L^{2} z \stackrel{(5.31)}{\leq} c \beta .
$$

Define $L:=L_{0} \cdot P_{0}^{-1}$, so $D L=D L_{0} \cdot D P_{0}^{-1}=R_{0} A_{0} \in K$ so $L$ satisfies (5.4) which completes the proof of Proposition 5.1.

Proposition 5.2. Let $w_{1} \in S^{1}$ be such that for some $w_{2} \in w_{1}^{\perp}$ we have that $w_{1}, w_{2}, \frac{w_{1}-w_{2}}{\left|w_{1}-w_{2}\right|}$ are not in the set of rank-1 directions connecting $S O(2) A_{i}$ to $S O(2) A_{j}$ for any $i \neq j$. Let $p \geq 1$, we will show that for some enough $\varsigma=\varsigma(\sigma)$ we can find $\tilde{u} \in \mathcal{D}_{F}^{\varsigma, \sqrt{\epsilon}}\left(w_{1}\right)$ such that

$$
\int_{\Omega} d^{p}(D \tilde{u}(z), K) \mathrm{d} L^{2} z \leq c m_{\epsilon}^{p} .
$$

Proof. The main idea for the proof is to take a function $u \in A_{F}$ with $I_{\epsilon}^{p}(u) \leq 2 m_{\epsilon}^{p}$ and to find a regular triangulation $\left\{\tau_{i}\right\} \in \triangle_{\sqrt{\epsilon}}^{\varsigma}\left(w_{1}\right)$ (recall notation from Sect. 1.1) such that when we define $\tilde{u}$ to be the piecewise affine interpolation of $u$ on $\left\{\tau_{i}\right\}$ then we have $\int_{\Omega} d^{p}(D \tilde{u}, K) \mathrm{d} L^{2} z \leq c m_{\epsilon}^{p}$ and $\tilde{u} \in \mathcal{D}_{F}^{\varsigma, \sqrt{\epsilon}}$. In order for $\tilde{u}$ to satisfy these properties we will need $D u$ to have controlled surface and bulk energies on the set $\bigcup_{i} \partial \tau_{i}$. 
However as $\left(\Omega \backslash N_{\varsigma^{-1} \sqrt{\epsilon}}(\partial \Omega)\right) \cap \bigcup_{i} \partial \tau_{i}$ is the intersection of three sets of evenly spaced parallel lines that are of order $O(\sqrt{\epsilon})$ apart, by applying the Co-area formula to all possible shifted copies of these sets of lines we can find a triangulation with the properties we want. The rest of the proof is just a matter of harvesting the inequalities we need.

Let $\mathcal{C}_{0}=\mathcal{C}_{0}(\sigma, \varsigma)$ be some small number we decide on later. We claim we can assume

$$
m_{\epsilon}^{p} \leq \mathcal{C}_{0}
$$

Suppose (5.33) is false, then we define $\tilde{u}=l_{F}$, clearly $l_{F} \in \mathcal{D}_{F}^{\varsigma, \sqrt{\epsilon}}$ and $\int_{\Omega} d^{p}\left(D l_{F}, K\right) \mathrm{d} L^{2} z \leq c$, so inequality (5.32) is satisfied. So we can assume (5.33) or there is nothing to show.

Let $u \in A_{F}$ be such that $I_{\epsilon}^{p}(u) \leq c m_{\epsilon}^{p}$. So we $\int_{\Omega}\left|D^{2} u(z)\right|^{2} \mathrm{~d} L^{2} z \leq c \epsilon^{-1} m_{\epsilon}^{p}$. Define $v(z):=\frac{u(\sqrt{\epsilon} z)}{\sqrt{\epsilon}}$. Recall $\Omega_{\epsilon^{-\frac{1}{2}}}=\epsilon^{-\frac{1}{2}} \Omega$. Note

and

$$
\int_{\Omega_{\epsilon}-\frac{1}{2}} d^{p}(D v(z), K) \mathrm{d} L^{2} z \leq c \epsilon^{-1} m_{\epsilon}^{p}
$$

$$
\int_{\Omega_{\epsilon^{-\frac{1}{2}}}}\left|D^{2} v(z)\right|^{2} \mathrm{~d} L^{2} z \leq c \epsilon^{-1} m_{\epsilon}^{p} .
$$

Let $T_{t}^{1}:=\left\{k w_{2}+\left\langle w_{1}\right\rangle: k \in \mathbb{Z}\right\}+t w_{2}$ and $T_{t}^{2}:=\left\{k w_{1}+\left\langle w_{2}\right\rangle: k \in \mathbb{Z}\right\}+t w_{1}$. Define $\mathbb{L}_{1}: \Omega_{\epsilon^{-\frac{1}{2}}} \rightarrow[0,1]$ to be such that $\mathbb{L}_{1}^{-1}(s)=T_{s}^{1} \cap \Omega_{\epsilon^{-\frac{1}{2}}}$ and $\mathbb{L}_{2}: \Omega_{\epsilon^{-\frac{1}{2}}} \rightarrow[0,1]$ to be such that $\mathbb{L}_{1}^{-1}(s)=T_{s}^{1} \cap \Omega_{\epsilon^{-\frac{1}{2}}}$. It is easy to see $\left|D \mathbb{L}_{1}\right| \leq 1,\left|D \mathbb{L}_{2}\right| \leq 1$.

Now $D v=F$ in the sense of trace on $\partial \Omega_{\epsilon^{-\frac{1}{2}}}$. By Theorem 2, Section 5.3 [17], this implies

$$
\lim _{r \rightarrow 0} f_{B_{r}(x) \cap \Omega_{\epsilon^{-\frac{1}{2}}}}|D v(z)-F(z)| \mathrm{d} L^{2} z=0 \text { for } H^{1} \text { a.e. } x \in \partial \Omega_{\epsilon^{-\frac{1}{2}}} .
$$

Let $\mathrm{S}_{1}, \ldots, \mathrm{S}_{p_{0}}$ denote the sides of $\partial \Omega_{\epsilon^{-\frac{1}{2}}}$. For simplicity we make the assumption that none of the sides $\mathrm{S}_{1}, \mathrm{~S}_{2}, \ldots, \mathrm{S}_{p_{0}}$ are parallel to $w_{1}, w_{2}$. Let $i \in\left\{1, \ldots, p_{0}\right\}$, there exists $\widetilde{\mathrm{S}}_{i} \subset \mathrm{S}_{i}$ with $L^{1}\left(\mathrm{~S}_{i} \backslash \widetilde{\mathrm{S}}_{i}\right)=0$ such that for any $x \in \widetilde{\mathrm{S}}_{i}$ we can find $r_{x} \in(0, \epsilon)$ with the property that for any $r \in\left(0, r_{x}\right]$ we have $\int_{B_{r}(x) \cap \Omega_{\epsilon}-\frac{1}{2}} \mid D v(z)-$ $F(z) \mid \mathrm{d} L^{2} z \leq \epsilon r^{2}$.

So there exists $\delta \in(0,1)$ such that for each $i$ we can find subset $\mathbb{S}_{i} \subset \widetilde{\mathrm{S}}_{i}$ with $L^{1}\left(\widetilde{\mathrm{S}}_{i} \backslash \mathbb{S}_{i}\right) \leq \epsilon$ and for each $x \in \mathbb{S}_{i}, r_{x} \geq \delta$.

Let $q \in\{1,2\}, i \in\left\{1, \ldots, p_{0}\right\}$. The set of intervals $\left\{P_{w_{q}^{\perp}}\left(B_{\delta}(x)\right): x \in \mathbb{S}_{i}\right\}$ forms a cover of $P_{w_{q}^{\perp}}\left(\mathbb{S}_{i}\right)$ and so by the $5 r$ Covering Theorem, Theorem $2.1[25]$ we can extract a subset $\left\{x_{1}, x_{2}, \ldots, x_{J_{0}}\right\} \subset \mathbb{S}_{i}$ such that

$$
\left\{P_{w_{q}^{\perp}}\left(B_{\frac{\delta}{5}}\left(x_{n}\right)\right): n \in\left\{1,2, \ldots, J_{0}\right\}\right\} \text { are disjoint }
$$

and

$$
P_{w_{q}^{\perp}}\left(\mathbb{S}_{i}\right) \subset \bigcup_{n=1}^{J_{0}} P_{w_{q}^{\perp}}\left(B_{\delta}\left(x_{n}\right)\right) .
$$

Let $C_{n}^{i}:=\left\{z \in B_{\delta}\left(x_{n}\right) \cap \Omega_{\epsilon^{-\frac{1}{2}}}:|D v(z)-F| \leq 1\right\}$ so $L^{2}\left(B_{\delta}\left(x_{n}\right) \backslash C_{n}^{i}\right) \leq \epsilon \delta^{2}$. This implies

$$
L^{1}\left(P_{w_{q}^{\perp}}\left(B_{\delta}\left(x_{n}\right)\right) \backslash P_{w_{q}^{\perp}}\left(\mathrm{C}_{n}^{i}\right)\right) \leq c \epsilon \delta
$$


Let $\Sigma_{i}=\bigcup_{n=1}^{J_{0}} C_{n}^{i}$. We have

$$
\begin{gathered}
L^{1}\left(P_{w_{q}^{\perp}}\left(\mathrm{S}_{i} \cap H\left(0, w_{q}\right)\right) \backslash P_{w_{q}^{\perp}}\left(\Sigma_{i} \cap H\left(0, w_{q}\right)\right)\right)=L^{1}\left(P_{w_{q}^{\perp}}\left(\mathrm{S}_{i} \cap H\left(0, w_{q}\right)\right) \backslash\left(\bigcup_{n=1}^{J_{0}} P_{w_{q}^{\perp}}\left(\mathrm{C}_{n}^{i} \cap H\left(0, w_{q}\right)\right)\right)\right) \\
\stackrel{(5.38),(5.39)}{\leq} c J_{0} \epsilon \delta \\
\quad \leq c \epsilon .37)
\end{gathered}
$$

By exactly the same argument

$$
L^{1}\left(P_{w_{q}^{\perp}}\left(\mathrm{S}_{i} \cap H\left(0,-w_{q}\right)\right) \backslash P_{w_{q}^{\perp}}\left(\Sigma_{i} \cap H\left(0,-w_{q}\right)\right)\right) \leq c \epsilon .
$$

Define

$$
\mathrm{A}_{0}:=\bigcup_{i=1}^{p_{0}} \Sigma_{i} \text { and note that } \mathrm{A}_{0} \subset N_{1}\left(\partial \Omega_{\epsilon^{-\frac{1}{2}}}\right) .
$$

Let $q \in\{1,2\}$ and let $l$ be such that $\{l\}=\{1,2\} \backslash\{q\}$. As shown on Figure 3, let

$$
Q_{1}^{q}=\inf \left\{k \in \mathbb{Z}:\left(k w_{l}+\left\langle w_{q}\right\rangle\right) \cap \Omega_{\epsilon^{-\frac{1}{2}}} \neq \emptyset\right\}
$$

and let

$$
Q_{2}^{q}=\sup \left\{k \in \mathbb{Z}:\left(k w_{l}+\left\langle w_{q}\right\rangle\right) \cap \Omega_{\epsilon^{-\frac{1}{2}}} \neq \emptyset\right\}
$$

Step 1. For $q \in\{1,2\}$ and $l$ be such that $\{l\}=\{1,2\} \backslash\{q\}$ define

$$
\mathrm{P}_{q}^{+}:=\left\{t \in[0,1]:\left(w_{q} \mathbb{R}_{+}+(t+k) w_{l}\right) \cap \mathrm{A}_{0} \neq \emptyset \text { for every } k \in\left\{Q_{1}^{q}, Q_{1}^{q}+1, \ldots, Q_{2}^{q}-1\right\}\right\}
$$

and

$$
\mathrm{P}_{q}^{-}:=\left\{t \in[0,1]:\left(w_{q} \mathbb{R}_{-}+(t+k) w_{l}\right) \cap \mathrm{A}_{0} \neq \emptyset \text { for every } k \in\left\{Q_{1}^{q}, Q_{1}^{q}+1, \ldots, Q_{2}^{q}-1\right\}\right\}
$$

we will show $L^{1}\left([0,1] \backslash \mathrm{P}_{q}^{+}\right) \leq c \sqrt{\epsilon}$ and $L^{1}\left([0,1] \backslash \mathrm{P}_{q}^{-}\right) \leq c \sqrt{\epsilon}$.

Proof of Step 1. We argue only for the set $\mathrm{P}_{1}^{+}$. For each $t \in[0,1] \backslash \mathrm{P}_{1}^{+}$let

$$
N_{t}:=\left\{k:\left(w_{1} \mathbb{R}_{+}+(t+k) w_{2}\right) \cap \mathrm{A}_{0}=\emptyset, k \in\left\{Q_{1}^{1}, Q_{1}^{1}+1, \ldots, Q_{2}^{1}-1\right\}\right\}
$$

and $l t^{6} n(t):=\min N_{t}$.

So $[0,1] \backslash P_{1}^{+}=\bigcup_{k \in\left\{Q_{1}^{1}, Q_{1}^{1}+1, \ldots, Q_{2}^{1}-1\right.} n^{n^{-1}}(k)$ and thus there must exist $k_{0}$ such that

$$
\begin{aligned}
L^{1}\left(n^{-1}\left(k_{0}\right)\right) & \geq \frac{L^{1}\left([0,1] \backslash \mathrm{P}_{1}^{+}\right)}{\left|Q_{1}^{1}\right|+\left|Q_{2}^{1}\right|} \\
& \geq \frac{\sqrt{\epsilon}}{5} L^{1}\left([0,1] \backslash \mathrm{P}_{1}^{+}\right) .
\end{aligned}
$$

However by definition since for every $t \in n^{-1}\left(k_{0}\right), k_{0}=n(t) \in N_{t}$ and by (5.45) we have

$$
\left(w_{1} \mathbb{R}_{+}+\left(t+k_{0}\right) w_{2}\right) \cap \mathrm{A}_{0}=\emptyset \text { for any } t \in n^{-1}\left(k_{0}\right)
$$

\footnotetext{
${ }^{6}$ We define $n(t)$ to be the minimum only to produce a well defined function, we could just as well take the maximum.
} 


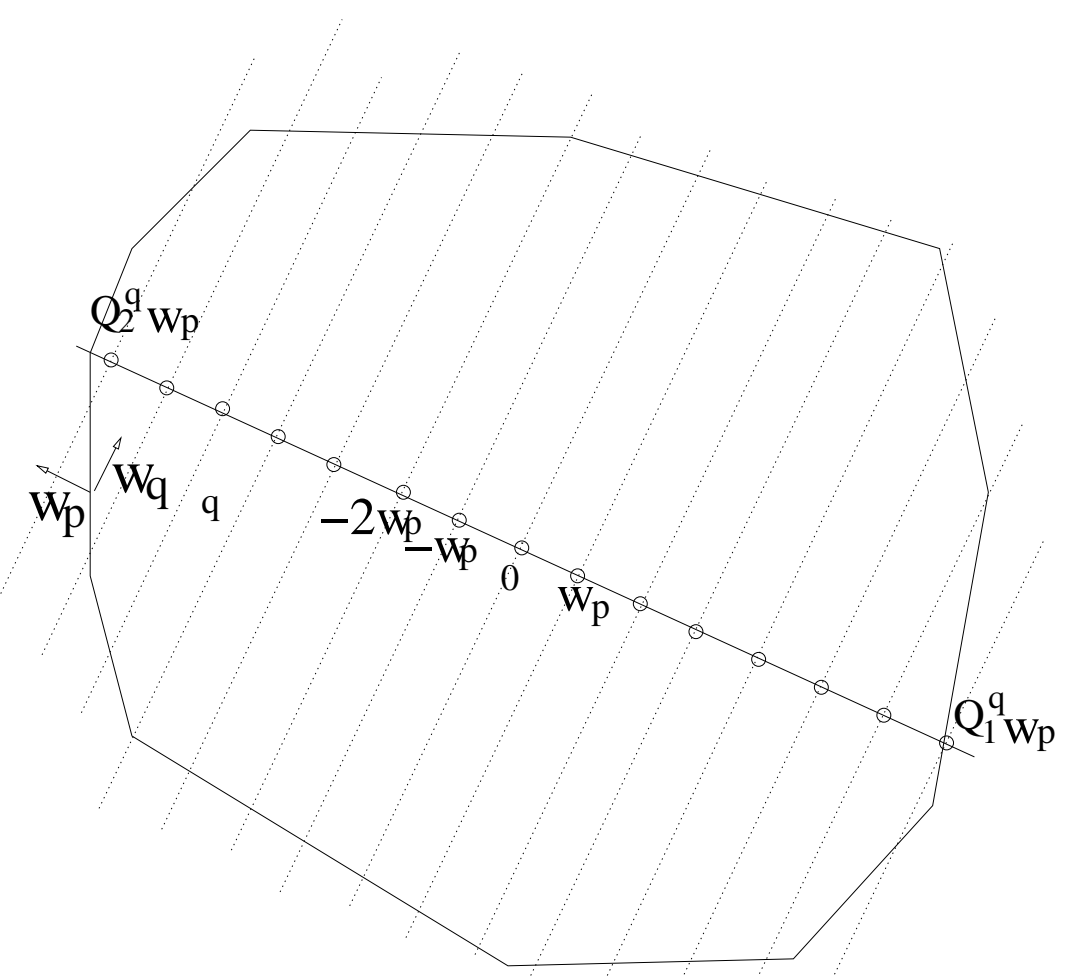

Figure 3. Constructing the triangulation for a typical polygonal region.

hence $\left(\left(t+k_{0}\right) w_{2}\right) \cap P_{w_{1}^{\perp}}\left(\mathrm{A}_{0} \cap H\left(0, w_{1}\right)\right)=\emptyset$ for any $t \in n^{-1}\left(k_{0}\right)$, i.e.

$$
\left(\left(n^{-1}\left(k_{0}\right)+k_{0}\right) w_{2}\right) \cap P_{w_{1}^{\perp}}\left(\mathrm{A}_{0} \cap H\left(0, w_{1}\right)\right)=\emptyset .
$$

Since $k_{0} \in\left\{Q_{1}^{1}, Q_{1}^{1}+1, \ldots, Q_{2}^{1}-1\right\}$ we have $\left(n^{-1}\left(k_{0}\right)+k_{0}\right) w_{2} \subset P_{w_{1}^{\perp}}\left(\Omega_{\epsilon^{-\frac{1}{2}}}\right)=P_{w_{1}^{\perp}}\left(\partial \Omega_{\epsilon^{-\frac{1}{2}}}\right)$ and by convexity of $\Omega$ this implies $\left(n^{-1}\left(k_{0}\right)+k_{0}\right) w_{2} \subset P_{w_{1}^{\perp}}\left(\partial \Omega_{\epsilon^{-\frac{1}{2}}} \cap H\left(0, w_{1}\right)\right)$ so for some $a \in\left\{1,2, \ldots, p_{0}\right\}$ we must have

$$
L^{1}\left(P_{w_{1}^{\perp}}\left(\mathrm{S}_{a} \cap H\left(0, w_{1}\right)\right) \cap\left(\left(n^{-1}\left(k_{0}\right)+k_{0}\right) w_{2}\right)\right) \geq \frac{L^{1}\left(n^{-1}\left(k_{0}\right)\right)}{p_{0}}
$$

and by (5.48) (and recalling definition (5.42)) we have

$$
P_{w_{1}^{\perp}}\left(\mathrm{S}_{a} \cap H\left(0, w_{1}\right)\right) \cap\left(\left(n^{-1}\left(k_{0}\right)+k_{0}\right) w_{2}\right) \subset P_{w_{1}^{\perp}}\left(\mathrm{S}_{a} \cap H\left(0, w_{1}\right)\right) \backslash P_{w_{1}^{\perp}}\left(\Sigma_{a} \cap H\left(0, w_{1}\right)\right)
$$

and thus from $(5.40),(5.49)$ we have $c \epsilon \geq L^{1}\left(n^{-1}\left(k_{0}\right)\right)$ by $(5.46) c \sqrt{\epsilon} \geq L^{1}\left([0,1] \backslash \mathrm{P}_{1}^{+}\right)$, this completes the proof of Step 1.

Step 2. Let $\left\{c_{i}: i=1,2, \ldots, N_{0}\right\}$ be an ordering of the set of points

$$
\left\{k_{1} w_{1}+k_{2} w_{2}: k_{1}, k_{2} \in \mathbb{Z}, k_{1} w_{1}+k_{2} w_{2} \in \Omega_{\epsilon^{-\frac{1}{2}}} \backslash N_{32 \sigma^{-2}}\left(\partial \Omega_{\epsilon^{-\frac{1}{2}}}\right)\right\} .
$$


Let $\mathcal{C}_{1}$ be some small positive number we decide on later. Let

$$
B_{1}:=\left\{i \in\left\{1,2, \ldots, N_{0}\right\}: \int_{B_{32 \sigma^{-2}}\left(c_{i}\right)}\left|D^{2} v(z)\right|^{2} \mathrm{~d} L^{2} z>\mathcal{C}_{1}\right\}
$$

and

$$
B_{2}:=\left\{i \in\left\{1,2, \ldots, N_{0}\right\}: \int_{B_{32 \sigma^{-2}\left(c_{i}\right)}} d^{p}(D v(z), K) \mathrm{d} L^{2} z>\mathcal{C}_{1}\right\}
$$

Note

$$
\operatorname{Card}\left(B_{1}\right)+\operatorname{Card}\left(B_{2}\right) \stackrel{(5.34),(5.35)}{\leq} c \epsilon^{-1} m_{\epsilon}^{p} .
$$

Define $G_{0}=\left\{1,2, \ldots, N_{0}\right\} \backslash\left(B_{1} \cup B_{2}\right)$. For the case $p=1$, for each $i \in G_{0}$ by Proposition 5.1 we have the existence of $q(i) \in\{1,2, \ldots, N\}$ and an affine function $L_{i}: B_{32}\left(c_{i}\right) \rightarrow \mathbb{R}^{2}$ with $D L_{i} \in S O(2) A_{q(i)}$ and

$$
\int_{B_{32}\left(c_{i}\right)}\left|v(z)-L_{i}(z)\right| \mathrm{d} L^{2} z \leq \int_{B_{32 \sigma-2}\left(c_{i}\right)} d(D v(z), K)+\left|D^{2} v(z)\right|^{2} \mathrm{~d} L^{2} z
$$

and

$$
\int_{B_{32}\left(c_{i}\right)} d\left(D v(z), S O(2) A_{q(i)}\right) \mathrm{d} L^{2} z \leq \int_{B_{32 \sigma-2}\left(c_{i}\right)} d(D v(z), K)+\left|D^{2} v(z)\right|^{2} \mathrm{~d} L^{2} z .
$$

For $p>1$ for each $i \in G_{0}$ by Proposition 5.1 we have a matrix $M_{i} \in K$ such that

$$
\int_{B_{32 \sigma^{-2}\left(c_{i}\right)}}\left|D v(z)-M_{i}\right|^{p} \mathrm{~d} L^{2} z \leq \int_{B_{32 \sigma^{-2}\left(c_{i}\right)}} d^{p}(D v(z), K)+\left|D^{2} v(z)\right|^{2} \mathrm{~d} L^{2} z .
$$

Define

$$
P(z)= \begin{cases}\sum_{i \in G_{0}} \chi_{B_{32}\left(c_{i}\right)}\left(\left|v(z)-L_{i}(z)\right|+d\left(D v(z), S O(2) A_{q(i)}\right)\right), & \text { if } p=1 \\ 0, & \text { if } p \in(1,2]\end{cases}
$$

and define

$$
Q(z)=\left\{\begin{array}{lc}
\sum_{i \in G_{0}} \chi_{B_{32}\left(c_{i}\right)}\left|D v(z)-M_{i}\right|^{p}, & \text { if } p \in(1,2] \\
0, & \text { if } p=1
\end{array}\right.
$$

Note

$$
\int_{\Omega_{\epsilon}^{-\frac{1}{2}}} Q(z)+P(z) \mathrm{d} L^{2} z \leq c \epsilon^{-1} m_{\epsilon}^{p} .
$$

By the Co-area formula we can find $\sigma_{1} \in \mathrm{P}_{1}^{+} \cap \mathrm{P}_{1}^{-}$and $\sigma_{2} \in \mathrm{P}_{2}^{+} \cap \mathrm{P}_{2}^{-}$such that

$$
\int_{\mathbb{L}_{i}^{-1}\left(\sigma_{i}\right)} d^{p}(D v(z), K)+\left|D^{2} v(z)\right|^{2} \mathrm{~d} H^{1} z \leq c \epsilon^{-1} m_{\epsilon}^{p} \text { for } i=1,2
$$

and

Now set

$$
\int_{\mathbb{L}_{i}^{-1}\left(\sigma_{i}\right)} P(z)+Q(z) \mathrm{d} H^{1} z \leq c \epsilon^{-1} m_{\epsilon}^{p} \text { for } i=1,2 .
$$

$$
\mathfrak{A}:=\Omega_{\epsilon^{-\frac{1}{2}}} \backslash\left(\mathbb{L}_{1}^{-1}\left(\sigma_{1}\right) \cup \mathbb{L}_{2}^{-1}\left(\sigma_{2}\right)\right) .
$$

Let $\mathcal{R}_{1}, \mathcal{R}_{2}, \ldots, \mathcal{R}_{N_{1}}$ denote those among them that form complete squares. Let $\left\{\tau_{1}, \tau_{2}, \ldots, \tau_{2 N_{1}}\right\}$ be a collection of right angle triangles with $\overline{\tau_{i}} \cup \overline{\tau_{i+N_{1}}}=\overline{\mathcal{R}_{i}}$ for each $i=1,2, \ldots, N_{1}$. 
Let

$$
G_{1}:=\left\{i \in\left\{1,2, \ldots, N_{1}\right\}: \overline{\mathcal{R}_{i}} \cap\left\{c_{i}: i \in G_{0}\right\} \neq \emptyset\right\} .
$$

Note that from (5.52) we have

$$
\operatorname{Card}\left(G_{1}\right) \geq N_{1}-c \epsilon^{-1} m_{\epsilon}^{p}
$$

For each $i \in\left\{1,2, \ldots, N_{1}\right\}$ let $l_{i}$ denote the affine function we obtain from interpolation of $v$ on the corners of $\tau_{i}$. We will show

$$
\sum_{i \in G_{1}} d^{p}\left(D l_{i}, K\right)+d^{p}\left(D l_{i+N_{1}}, K\right) \leq c \epsilon^{-1} m_{\epsilon}^{p} .
$$

Proof of Step 2. Case $p>1$. Firstly we will deal with the simpler case.

For any $i \in G_{1}, \tau_{i}$ has two sides parallel to $w_{1}, w_{2}$. Let $\{a, b, e\}$ denote the corners of $\tau_{i}$ where we have order them so that $\frac{a-b}{|a-b|}=w_{1}$ and $\frac{e-b}{|e-b|}=w_{2}$.

$$
\begin{gathered}
\left|D l_{i} w_{1}-M_{i} w_{1}\right| \stackrel{=}{=}|a-b|^{-1}\left|\int_{[a, b]}\left(D v(z)-M_{i}\right) w_{1} \mathrm{~d} H^{1} z\right| \\
\stackrel{(5.57)}{\leq} c\left(\int_{[a, b]} Q(z) \mathrm{d} H^{1} z\right)^{\frac{1}{p}} .
\end{gathered}
$$

So $\left|D l_{i} w_{1}-M_{i} w_{1}\right|^{p} \leq c \int_{[a, b]} Q(z) \mathrm{d} H^{1} z$, in the same way $\left|D l_{i} w_{2}-M_{i} w_{2}\right|^{p} \leq c \int_{[b, e]} Q(z) \mathrm{d} H^{1} z$.

Assume without loss of generality $\left|D l_{i} w_{1}-M_{i} w_{1}\right| \leq\left|D l_{i} w_{2}-M_{i} w_{2}\right|$ so

$$
\left|D l_{i}-M_{i}\right|^{p} \leq c \int_{\partial \mathcal{R}_{i}} Q(z) \mathrm{d} H^{1} z .
$$

So $d^{p}\left(D l_{i}, K\right) \leq c \int_{\partial \mathcal{R}_{i}} Q(z) \mathrm{d} H^{1} z$ in exactly the same we have $d^{p}\left(D l_{i+N_{1}}, K\right) \leq c \int_{\partial \mathcal{R}_{i}} Q(z) \mathrm{d} H^{1} z$. Thus

$$
\sum_{i \in G_{1}} d^{p}\left(D l_{i}, K\right)+d^{p}\left(D l_{i+N_{1}}, K\right) \leq c \int_{\mathbb{L}^{-1}\left(\sigma_{1}\right) \cup \mathbb{L}^{-1}\left(\sigma_{2}\right)} Q(z) \mathrm{d} H^{1} z \leq c \epsilon^{-1} m_{\epsilon}^{p}
$$

Case $p=1$. Now we tackle the more difficult case. Let $i \in G_{1}$. So there exists $p(i) \in G_{0}$ such that $c_{p(i)} \cap \overline{\mathcal{R}_{i}} \neq \emptyset$. Let

$$
\alpha_{i}=\int_{\partial \mathcal{R}_{i}} P(z)+|D v(z)|^{2} \mathrm{~d} H^{1} z+\int_{B_{32 \sigma^{-2}}\left(c_{p(i)}\right)} d(D v(z), K)+P(z)+\left|D^{2} v(z)\right|^{2} \mathrm{~d} L^{2} z
$$

So there exists $R_{p(i)} \in S O(2)$ such that $D L_{p(i)}=R_{p(i)} A_{s(i)}$ for some $s(i) \in\{1,2, \ldots, N\}$ (note that $s(i)=$ $q(p(i))$, see (5.54)). Let $\{a, b, d, e\}$ denote that corners of $\mathcal{R}_{i}$ where $\frac{a-b}{|a-b|}=w_{1}, \frac{e-b}{|e-b|}=w_{2}$.

By definition of $\alpha_{i}$ there exists $x_{1}, x_{2} \in[a, b],\left|x_{1}-x_{2}\right|>c, P\left(x_{1}\right) \leq c \alpha_{i}$ and $P\left(x_{2}\right) \leq c \alpha_{i}$. So

$$
\left|v\left(x_{1}\right)-L_{p(i)}\left(x_{1}\right)\right| \leq c \alpha_{i},\left|v\left(x_{2}\right)-L_{p(i)}\left(x_{2}\right)\right| \leq c \alpha_{i}
$$

thus

$$
\left|v\left(x_{1}\right)-v\left(x_{2}\right)-R_{p(i)} A_{s(i)}\left(x_{1}-x_{2}\right)\right| \leq c \alpha_{i} .
$$

Since $\int_{[a, b]}\left|D^{2} v(z)\right| \mathrm{d} H^{1} z \leq c \sqrt{\alpha_{i}}$ there exists $R_{0}$ such that

$$
\sup \left\{\left|D v(z)-R_{0} A_{s(i)}\right|: z \in[a, b]\right\} \leq c \sqrt{\alpha_{i}} .
$$


So

$$
\begin{aligned}
& \left|v\left(x_{1}\right)-v\left(x_{2}\right)-R_{0} A_{s(i)}\left(x_{1}-x_{2}\right)\right|=\left|\int_{\left[x_{1}, x_{2}\right]}\left(D v(z)-R_{0} A_{s(i)}\right) \frac{x_{1}-x_{2}}{\left|x_{1}-x_{2}\right|} \mathrm{d} H^{1} z\right| \\
& \stackrel{(5.67)}{\leq} c \sqrt{\alpha_{i}} \text {. }
\end{aligned}
$$

Putting this together with (5.66) gives

$$
\left|R_{0}-R_{p(i)}\right| \leq c \sqrt{\alpha_{i}} .
$$

For $z \in[a, b]$ define $R(z) \in S O(2)$ be such that $d\left(D v(z), S O(2) A_{s(i)}\right)=\left|D v(z)-R(z) A_{s(i)}\right|$. So note that $\int_{[a, b]} d(R(z), S O(2)) \mathrm{d} H^{1} z \leq c \alpha_{i}$. Note also that from (5.67) and (5.68) we have

$$
\sup \left\{\left|R(z)-R_{p(i)}\right|: z \in[a, b]\right\} \leq c \sqrt{\alpha_{i}} \text {. }
$$

Arguing as in Step 3, Proposition 5.1. Let $\theta, \theta(z) \in[0,2 \pi)$ so that $R(z)=\left(\begin{array}{cc}\sin \theta(z) & -\cos \theta(z) \\ \cos \theta(z) & \sin \theta(z)\end{array}\right)$ and $R=\left(\begin{array}{cc}\sin \theta & -\cos \theta \\ \cos \theta & \sin \theta\end{array}\right)$. We have

$$
\begin{aligned}
R(z) e_{1} \cdot R e_{1} & =\cos (\theta(z)-\theta) \\
& \stackrel{(5.69)}{\geq} \\
& 1-c \alpha_{i} \text { for any } z \in[a, b] .
\end{aligned}
$$

We can pick point $\tilde{a} \in[a, b]$ with $|b-\tilde{a}|>c$ and $\tilde{e} \in[b, e]$ with $|\tilde{e}-b|>c$ where

$$
\left|v(\tilde{e})-L_{p(i)}(\tilde{e})\right| \leq c \alpha_{i} \text { and }\left|v(\tilde{a})-L_{p(i)}(\tilde{a})\right| \leq c \alpha_{i} .
$$

Let $\gamma_{1}=|\tilde{a}-b|\left|A_{s(i)} w_{1}\right|$ and $\gamma_{2}=|\tilde{e}-b|\left|A_{s(i)} w_{2}\right|$. We claim

$$
v(b) \in N_{c \alpha_{i}}\left(\partial B_{\gamma_{1}}(v(\tilde{a}))\right)
$$

and

To see this note that

$$
v(b) \in N_{c \alpha_{i}}\left(\partial B_{\gamma_{2}}(v(\tilde{e}))\right) .
$$

$$
\begin{aligned}
& \left|(v(\tilde{a})-v(b)) \cdot R_{p(i)} A_{s(i)}\left(-w_{1}\right)\right| \geq\left|A_{s(i)} w_{1}\right|^{2}\left|\int_{[\tilde{a}, b]} R(z) e_{1} \cdot R_{p(i)} e_{1} \mathrm{~d} H^{1} z\right|-c \alpha_{i} \\
& \stackrel{(5.70)}{\geq}\left|A_{s(i)} w_{1}\right|^{2}|\tilde{a}-b|\left(1-c \alpha_{i}\right)
\end{aligned}
$$

which implies $|v(\tilde{a})-v(b)| \geq\left|A_{s(i)} w_{1}\right||\tilde{a}-b|\left(1-c \alpha_{i}\right)=\gamma_{1}-c \alpha_{i}$. Now

$$
\begin{aligned}
|v(\tilde{a})-v(b)| & \leq\left|\int_{[\tilde{a}, b]}-R(z) A_{i_{0}} w_{1} \mathrm{~d} H^{1} z\right|+c \alpha_{i} \\
& \leq \gamma_{1}+c \alpha_{i}
\end{aligned}
$$

which establishes (5.72). Inclusion (5.73) can be shown in exactly the same way. So putting (5.71) together with (5.72), (5.73) we have established that

$$
v(b) \in N_{c \alpha_{i}}\left(\partial B_{\gamma_{1}}\left(L_{p(i)}(\tilde{a})\right)\right) \cap N_{c \alpha_{i}}\left(\partial B_{\gamma_{2}}\left(L_{p(i)}(\tilde{e})\right)\right) .
$$




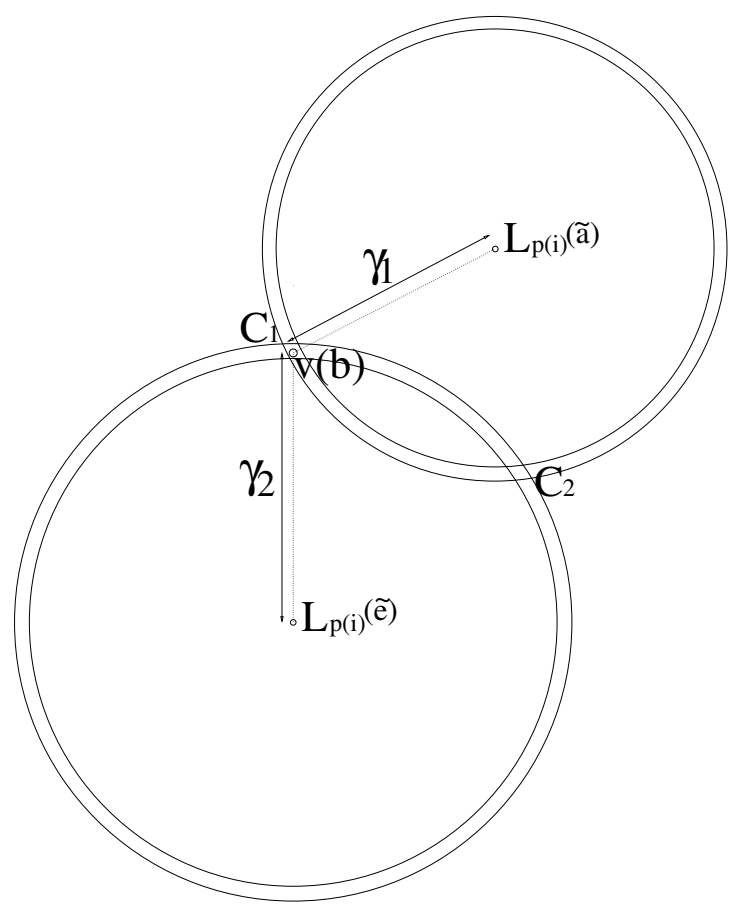

Figure 4. Controlling the function of the corners of a triangle.

Now the set $N_{c \alpha_{i}}\left(\partial B_{\gamma_{1}}\left(L_{p(i)}(\tilde{a})\right)\right) \cap N_{c \alpha_{i}}\left(\partial B_{\gamma_{2}}\left(L_{p(i)}(\tilde{e})\right)\right)$ consists of two disjoint connected components which we denote $C_{1}$ and $C_{2}$, see Figure 4 . It is quite straightforward to see that $\operatorname{diam}\left(C_{i}\right) \leq c \alpha_{i}$ for $i=1,2$.

Let $C_{1}$ be the component that contains $L_{p(i)}(b)$. We will show $v(b) \in C_{1}$. We argue by contradiction, suppose $v(b) \in C_{2}$. By Proposition 5.1, inequality (5.5) (recall $s(i)=q(p(i))$ ) we know

$$
\int_{B_{32}\left(c_{p(i)}\right)} d\left(D v(z), S O(2) A_{s(i)}\right) \mathrm{d} L^{2} z \stackrel{(5.34),(5.35)}{\leq} c \alpha_{i} .
$$

So by Proposition $2.6[9]$ we have that there exists $R_{0} \in S O(2)$ such that

$$
\int_{B_{32}\left(c_{p(i)}\right)}\left|D v(z)-R_{0} A_{s(i)}\right| \mathrm{d} L^{2} z \leq c \log \left(\alpha_{i}^{-1}\right) \alpha_{i} .
$$

Now by Sobolev embedding theorem there exists matrix $M_{i}$ such that

$$
\left(\int_{B_{32}\left(c_{p(i)}\right)}\left|D v(z)-M_{i}\right|^{3} \mathrm{~d} L^{2} z\right)^{\frac{1}{3}} \leq c\left(\int_{B_{32}\left(c_{p(i)}\right)}\left|D^{2} v(z)\right|^{2} \mathrm{~d} L^{2} z\right)^{\frac{1}{2}} \leq c \sqrt{\alpha_{i}} .
$$

So

$$
\left|M_{i}-R_{0} A_{s(i)}\right| \stackrel{(5.74),(5.75)}{\leq} c \sqrt{\alpha_{i}} .
$$


Let $\Lambda_{i}: B_{32}\left(c_{p(i)}\right) \rightarrow \mathbb{R}^{2}$ be such that $D \Lambda_{i}=R_{0} A_{s(i)}$ and $\Lambda_{i}(0)=0$. Define $w_{i}(z)=\Lambda_{i}(z)+$ $f_{B_{32}\left(c_{p(i)}\right)} v(x)-\Lambda_{i}(x) \mathrm{d} L^{2} x$ so

$$
f_{B_{32}\left(c_{p(i)}\right)} v(z)-w_{i}(z) \mathrm{d} L^{2} z=0
$$

and

$$
\begin{aligned}
& \left(\int_{B_{32}\left(c_{p(i)}\right)}\left|D v(z)-D w_{i}\right|^{3} \mathrm{~d} L^{2} z\right)^{\frac{1}{3}} \leq\left(\int_{B_{32}\left(c_{p(i)}\right)}\left|D v(z)-M_{i}\right|^{3} \mathrm{~d} L^{2} z\right)^{\frac{1}{3}} \\
& \begin{array}{ll}
\stackrel{(5.76),(5.75)}{\leq} & +c\left|M_{i}-R_{0} A_{s(i)}\right| \\
\leq & c \sqrt{\alpha_{i}} .
\end{array}
\end{aligned}
$$

So by Morrey's inequality Theorem 3, Section 4.5.3 [17], together with (5.77) this implies

$$
\left\|v-w_{i}\right\|_{L^{\infty}\left(B_{32}\left(c_{p(i)}\right)\right)} \leq c \sqrt{\alpha_{i}} .
$$

Since (5.53), (5.65) $\left\|v-L_{p(i)}\right\|_{L^{1}\left(B_{32}\left(c_{p(i)}\right)\right)} \leq c \alpha_{i}$ we have $\left\|w_{i}-L_{p(i)}\right\|_{L^{1}\left(B_{32}\left(c_{p(i)}\right)\right)} \leq c \sqrt{\alpha_{i}}$. And since $w_{i}$ and $L_{p(i)}$ are both affine this implies $\left|D w_{i}-D L_{p(i)}\right| \leq c \sqrt{\alpha_{i}}$ and thus $\left\|w_{i}-L_{p(i)}\right\|_{L^{\infty}\left(B_{32}\left(c_{p(i)}\right)\right)} \leq c \sqrt{\alpha_{i}}$. Putting this together with (5.78) we have that

$$
\left\|v-L_{p(i)}\right\|_{L^{\infty}\left(B_{32}\left(c_{p(i)}\right)\right)} \leq c \sqrt{\alpha_{i}} .
$$

Recall we are arguing by contradiction, as we supposed $v(b) \in C_{2}$, from (5.79) this implies that $L_{p(i)}(b) \in$ $N_{c \sqrt{\alpha}}\left(C_{2}\right)$ however as we also know $L_{p(i)}(b) \in C_{1}$ and $d\left(C_{1}, C_{2}\right)>c$ this is a contradiction.

Thus we have that

$$
v(b) \in C_{1} \subset B_{c \alpha_{i}}\left(L_{p(i)}(b)\right) .
$$

Arguing in exactly the same way we can establish the same thing for the other corners of $\mathcal{R}_{i}$, i.e. we can show

$$
v(a) \in B_{c \alpha_{i}}\left(L_{p(i)}(a)\right), v(d) \in B_{c \alpha_{i}}\left(L_{p(i)}(d)\right), v(e) \in B_{c \alpha_{i}}\left(L_{p(i)}(e)\right) .
$$

Recall $l_{i}$ and $l_{i+N_{1}}$ are the affine maps we obtained from interpolating $v$ on the corners of triangle $\tau_{i}$ and $\tau_{i+N_{1}}$ where $\overline{\tau_{i}} \cup \overline{\tau_{i+N_{1}}}=\overline{\mathcal{R}_{i}}$. Recall also that $D L_{p(i)}=R_{p(i)} A_{s(i)}$ where $R_{p(i)} \in S O(2), s(i) \in\{1,2, \ldots, N\}$. From (5.80) and (5.81) we have

$$
\begin{aligned}
\left|D l_{i} w_{1}-R_{p(i)} A_{s(i)} w_{1}\right| & =\left|\left(\frac{v(a)-v(b)}{|a-b|}\right)-\left(\frac{L_{p(i)}(a-b)}{|a-b|}\right)\right| \\
& \leq c \alpha_{i} .
\end{aligned}
$$

In the same way we can show $\left|D l_{i} w_{2}-R_{p(i)} A_{s(i)} w_{2}\right| \leq c \alpha_{i}$ which gives $\left|D l_{i}-R_{p(i)} A_{s(i)}\right| \leq c \alpha_{i}$ and hence $d\left(D l_{i}, K\right) \leq c \alpha_{i}$. In exactly the same way we can show $d\left(D l_{i+N}, K\right) \leq c \alpha_{i}$. 
Thus using (5.34), (5.35), (5.58), (5.59) and (5.60) for the last inequality

$$
\begin{aligned}
\sum_{i \in G_{1}} d\left(D l_{i}, K\right) & +d\left(D l_{i+N_{1}}, K\right) \stackrel{(5.65)}{\leq} c \sum_{i \in G_{1}} \int_{\partial \mathcal{R}_{i}} P(z)+\left|D^{2} v(z)\right|^{2} \mathrm{~d} H^{1} z \\
& +c \int_{B_{32 \sigma^{-2}}\left(c_{p(i)}\right)} d(D v(z), K)+P(z)+\left|D^{2} v(z)\right|^{2} \mathrm{~d} L^{2} z \\
\leq & c \int_{\mathbb{L}_{1}^{-1}\left(\sigma_{1}\right) \cup \mathbb{L}_{2}^{-1}\left(\sigma_{2}\right)} P(z)+\left|D^{2} v(z)\right|^{2} \mathrm{~d} H^{1} z \\
& +c \sum_{i \in G_{0}} \int_{B_{32 \sigma^{-2}\left(c_{i}\right)}} d(D v(z), K)+P(z)+\left|D^{2} v(z)\right|^{2} \mathrm{~d} L^{2} z \\
\leq & c \epsilon^{-1} m_{\epsilon}^{1} .
\end{aligned}
$$

Thus we have shown (5.64) in the case $p=1$. This completes the proof of Step 2.

Step 3. We will show

$$
\sum_{i \in\left\{1,2, \ldots, N_{1}\right\}} d^{p}\left(D l_{i}, K\right)+d^{p}\left(D l_{i+N}, K\right) \leq c \epsilon^{-1} m_{\epsilon}^{p} .
$$

Proof of Step 3. Let $i \in\left\{1,2, \ldots, N_{1}\right\} \backslash G_{1}$ and let $\left\{a_{i}, b_{i}, c_{i}\right\}$ denote the corners of $\tau_{i}$ where we have ordered them so that $\frac{a_{i}-b_{i}}{\left|a_{i}-b_{i}\right|}=w_{1}$ and $\frac{c_{i}-b_{i}}{\left|c_{i}-b_{i}\right|}=w_{2}$. Let $D l_{i}$ denote the affine map we obtain from interpolation of $v$ on the corners of $\tau_{i}$. Note

$$
\left|D l_{i} w_{1}\right|^{p}=\left|\frac{v\left(a_{i}\right)-v\left(b_{i}\right)}{\left|a_{i}-b_{i}\right|}\right|^{p} \leq c \int_{a_{i}}^{b_{i}}|D v(z)|^{p} \mathrm{~d} H^{1} z \leq c \int_{\partial \mathcal{R}_{i}} d^{p}(D v(z), K) \mathrm{d} H^{1} z+c .
$$

In exactly the same way we have $\left|D l_{i} w_{2}\right|^{p} \leq c \int_{\partial \mathcal{R}_{i}} d^{p}(D v(z), K) \mathrm{d} H^{1} z+c$ which gives

$$
\left|D l_{i}\right|^{p} \leq c \int_{\partial \mathcal{R}_{i}} d^{p}(D v(z), K) \mathrm{d} H^{1} z+c
$$

in exactly the same way $\left|D l_{i+N}\right|^{p} \leq c \int_{\partial \mathcal{R}_{i}} d^{p}(D v(z), K) \mathrm{d} H^{1} z+c$. As $d^{p}\left(D l_{i}, K\right) \leq c\left|D l_{i}\right|^{p}+c$ and $d^{p}\left(D l_{i+N}, K\right) \leq c\left|D l_{i+N}\right|^{p}+c$ thus

$$
\begin{array}{ll}
\sum_{i \in\left\{1,2, \ldots, N_{1}\right\} \backslash G_{1}} d^{p}\left(D l_{i}, K\right)+d^{p}\left(D l_{i+N}, K\right) \leq & \sum_{\substack{i \in\left\{1,2, \ldots, N_{1}\right\} \backslash G_{1}\\
}} c\left|D l_{i}\right|^{p}+c\left|D l_{i+N}\right|^{p} \\
& +c \operatorname{Card}\left(\left\{1,2, \ldots, N_{1}\right\} \backslash G_{1}\right) \\
& \\
& c \epsilon^{-1} m_{\epsilon}^{p} .
\end{array}
$$

Putting (5.83) together with (5.64) gives (5.82).

Step 4. Recall $\left\{\mathcal{R}_{1}, \mathcal{R}_{2}, \ldots, \mathcal{R}_{N_{1}}\right\}$ denote the connected components of $\mathfrak{A}$ (see (5.61)) that form complete squares, and $\left\{\tau_{1}, \tau_{2}, \ldots, \tau_{2 N_{1}}\right\}$ are triangles where $\overline{\tau_{i}} \cup \overline{\tau_{i+N_{1}}}=\overline{\mathcal{R}_{i}}$. Let

$$
V_{0}(i):=\left\{j \in\left\{1,2, \ldots, 2 N_{1}\right\}: H^{1}\left(\overline{\tau_{i}} \cap \overline{\tau_{j}}\right)>\varsigma\right\} .
$$

For any $j \in\left\{1,2, \ldots, 2 N_{1}\right\}$ let $l_{j}$ denote the affine map we get by interpolating $v$ on the corners of $\tau_{j}$. Define

$$
\Upsilon_{0}:=\left\{i \in\left\{1,2, \ldots, 2 N_{1}\right\}: \text { There exists } j \in V_{0}(i) \text { such that }\left|D l_{i}-D l_{j}\right|>\varsigma^{-1}\right\} \text {. }
$$


We will show

$$
\sum_{i \in \Upsilon_{0}} \sum_{j \in V_{0}(i)}\left|D l_{i}-D l_{j}\right|^{2} \leq c \epsilon^{-1} m_{\epsilon}^{p}
$$

Proof of Step 4. For any $i \in\left\{1,2, \ldots, 2 N_{1}\right\}$ define

$$
\rho(i):= \begin{cases}i & \text { if } i \in\left\{1,2, \ldots, N_{1}\right\} \\ i-N_{1} & \text { if } i \in\left\{N_{1}+1, \ldots, 2 N_{1}\right\} .\end{cases}
$$

To start we will show that if $i \in\left\{1,2, \ldots, 2 N_{1}\right\}$ and $j \in V_{0}(i)$ then

$$
\left|D l_{i}-D l_{j}\right| \leq c\left(\int_{\partial\left(\mathcal{R}_{\rho(i)} \cup \mathcal{R}_{\rho(j)}\right)}\left|D^{2} v(z)\right|^{2} \mathrm{~d} H^{1} z\right)^{\frac{1}{2}} .
$$

So see this we will argue as follows. Note $\overline{\mathcal{R}_{\rho(i)}} \cup \overline{\mathcal{R}_{\rho(j)}}$ forms a rectangle, thus $\overline{\tau_{i}} \cup \overline{\tau_{j}}$ must form a regular parallelogram with two opposite sides that intersect $\partial\left(\mathcal{R}_{\rho(i)} \cup \mathcal{R}_{\rho(j)}\right)$, see Figure 5 .

Let $U_{i}$ denote the side of $\partial \tau_{i}$ that intersects $\partial\left(\mathcal{R}_{\rho(i)} \cup \mathcal{R}_{\rho(j)}\right)$ and $U_{j}$ denote the side of $\partial \tau_{j}$ that intersects $\partial\left(\mathcal{R}_{\rho(i)} \cup \mathcal{R}_{\rho(j)}\right)$. Let $q \in\{1,2\}$ be such that $U_{i}$ and $U_{j}$ are parallel to $w_{q}$. Now by the fundamental theorem of Calculus (and Holder's inequality) there must exist $M \in M^{2 \times 2}$ such that

$$
\sup \left\{|D v(z)-M|: z \in \partial\left(\mathcal{R}_{\rho(i)} \cup \mathcal{R}_{\rho(j)}\right)\right\} \leq c\left(\int_{\partial\left(\mathcal{R}_{\rho(i)} \cup \mathcal{R}_{\rho(j)}\right)}\left|D^{2} v(z)\right|^{2} \mathrm{~d} H^{1} z\right)^{\frac{1}{2}} .
$$

Let $\left\{\omega_{1}^{i}, \omega_{2}^{i}, \omega_{3}^{i}\right\}$ denote the corners of $\tau_{i}$ and $\left\{\omega_{1}^{j}, \omega_{2}^{j}, \omega_{3}^{j}\right\}$ the corners of $\tau_{j}$ where we have chosen to label these points such that $\omega_{3}^{i}-\omega_{2}^{i}=\omega_{2}^{j}-\omega_{1}^{j}$ and $\omega_{1}^{i}=\omega_{2}^{j}, \omega_{2}^{i}=\omega_{3}^{j}$, see Figure 5 , note $\left\{\omega_{3}^{i}, \omega_{2}^{i}\right\}=\partial U_{i}$ and $\left\{\omega_{2}^{j}, \omega_{1}^{j}\right\}=\partial U_{j}$, again see Figure 5. Recall we know triangles $\tau_{i}, \tau_{j}$ are conjugate to each other and hence $\left|\omega_{3}^{i}-\omega_{2}^{i}\right|=\left|\omega_{2}^{j}-\omega_{1}^{j}\right|$. By definition

$$
D l_{i}\left(\frac{\omega_{3}^{i}-\omega_{2}^{i}}{\left|\omega_{3}^{i}-\omega_{2}^{i}\right|}\right)=\frac{l_{i}\left(\omega_{3}^{i}\right)-l_{i}\left(\omega_{2}^{i}\right)}{\left|\omega_{3}^{i}-\omega_{2}^{i}\right|}=\frac{v\left(\omega_{3}^{i}\right)-v\left(\omega_{2}^{i}\right)}{\left|\omega_{3}^{i}-\omega_{2}^{i}\right|}
$$

and in the same way

$$
D l_{j}\left(\frac{\omega_{2}^{j}-\omega_{1}^{j}}{\left|\omega_{2}^{j}-\omega_{1}^{j}\right|}\right)=\frac{v\left(\omega_{2}^{j}\right)-v\left(\omega_{1}^{j}\right)}{\left|\omega_{2}^{j}-\omega_{1}^{j}\right|} .
$$

Let $l_{M}$ denote an affine function with $D l_{M}=M$

$$
\begin{aligned}
&\left|v\left(\omega_{3}^{i}\right)-v\left(\omega_{2}^{i}\right)-l_{M}\left(\omega_{3}^{i}-\omega_{2}^{i}\right)\right| \leq \int_{\left[\omega_{3}^{i}, \omega_{2}^{i}\right]}|D v(z)-M| \mathrm{d} H^{1} z \\
& \stackrel{(5.88)}{\leq} c\left(\int_{\partial\left(\mathcal{R}_{\rho(i)} \cup \mathcal{R}_{\rho(j)}\right)}\left|D^{2} v(z)\right|^{2} \mathrm{~d} H^{1} z\right)^{\frac{1}{2}} .
\end{aligned}
$$

In the same way

$$
\left|v\left(\omega_{2}^{j}\right)-v\left(\omega_{1}^{j}\right)-l_{M}\left(\omega_{2}^{j}-\omega_{1}^{j}\right)\right| \leq c\left(\int_{\partial\left(\mathcal{R}_{\rho(i)} \cup \mathcal{R}_{\rho(j)}\right)}\left|D^{2} v(z)\right|^{2} \mathrm{~d} H^{1} z\right)^{\frac{1}{2}} .
$$




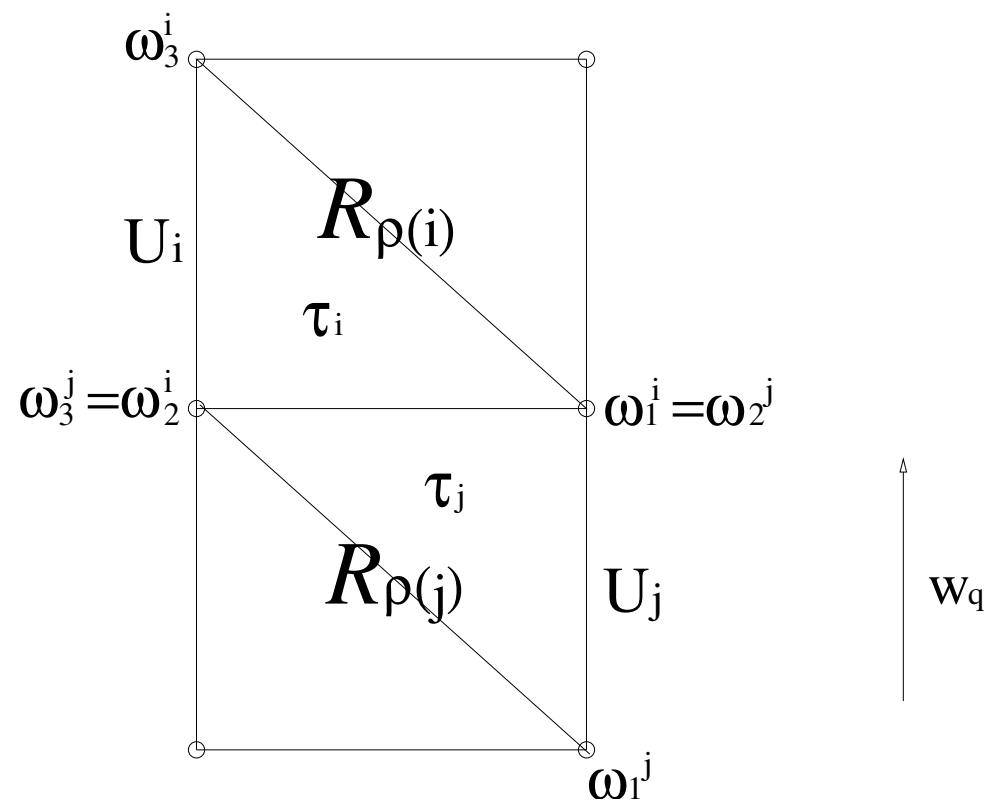

Figure 5. Two touching triangles.

Thus as $\omega_{2}^{j}-\omega_{1}^{j}=\omega_{3}^{i}-\omega_{2}^{i}$ (see Fig. 5) we have from (5.91), (5.92)

$$
\left|\frac{v\left(\omega_{3}^{i}\right)-v\left(\omega_{2}^{i}\right)}{\left|\omega_{3}^{i}-\omega_{2}^{i}\right|}-\frac{v\left(\omega_{2}^{j}\right)-v\left(\omega_{1}^{j}\right)}{\left|\omega_{2}^{j}-\omega_{1}^{j}\right|}\right| \leq c\left(\int_{\partial\left(\mathcal{R}_{\rho(i)} \cup \mathcal{R}_{\rho(j)}\right)}\left|D^{2} v(z)\right|^{2} \mathrm{~d} H^{1} z\right)^{\frac{1}{2}} .
$$

Which from (5.89) and (5.90) implies

$$
\left|D l_{i}\left(\frac{\omega_{3}^{i}-\omega_{2}^{i}}{\left|\omega_{3}^{i}-\omega_{2}^{i}\right|}\right)-D l_{j}\left(\frac{\omega_{3}^{i}-\omega_{2}^{i}}{\left|\omega_{3}^{i}-\omega_{2}^{i}\right|}\right)\right| \leq c\left(\int_{\partial\left(\mathcal{R}_{\rho(i)} \cup \mathcal{R}_{\rho(j)}\right)}\left|D^{2} v(z)\right|^{2} \mathrm{~d} H^{1} z\right)^{\frac{1}{2}} .
$$

Recall again (see Fig. 5) the endpoints of $\overline{\tau_{i}} \cap \overline{\tau_{j}}$ are given by $\omega_{1}^{i}, \omega_{2}^{i}$. So

$$
D l_{i}\left(\omega_{1}^{i}-\omega_{2}^{i}\right)=D l_{j}\left(\omega_{1}^{i}-\omega_{2}^{i}\right)
$$

and as $\frac{\omega_{1}^{i}-\omega_{2}^{i}}{\left|\omega_{1}^{i}-\omega_{2}^{i}\right|} \cdot \frac{\omega_{3}^{i}-\omega_{2}^{i}}{\left|\omega_{3}^{i}-\omega_{2}^{i}\right|}=0$ so (5.87) follows from (5.93) and (5.94). Thus

$$
\begin{aligned}
\sum_{i=1}^{2 N_{1}} \sum_{j \in V_{0}(i)}\left|D l_{i}-D l_{j}\right|^{2} & \stackrel{(5.87)}{\leq} \sum_{i=1}^{2 N_{1}} \sum_{j \in V_{0}(i)} \int_{\partial\left(\mathcal{R}_{\rho(i)} \cup \mathcal{R}_{\rho(j)}\right)}\left|D^{2} v(z)\right|^{2} \mathrm{~d} H^{1} z \\
& \leq c \int_{\mathbb{L}_{1}^{-1}\left(\sigma_{1}\right) \cup \mathbb{L}_{2}^{-1}\left(\sigma_{2}\right)}\left|D^{2} v(z)\right|^{2} \mathrm{~d} H^{1} z \\
& \stackrel{(5.59)}{\leq} c \epsilon^{-1} m_{\epsilon}^{p} .
\end{aligned}
$$


Step 5. Recall $\mathcal{R}_{1}, \mathcal{R}_{2}, \ldots, \mathcal{R}_{N_{1}}$ are the connected component of $\mathfrak{A}$ (see (5.61)). Let $\mathcal{D}_{1}, \mathcal{D}_{2}, \ldots, \mathcal{D}_{N_{2}}$ denote the connected components of

$$
\left(\Omega_{\epsilon^{-\frac{1}{2}}} \backslash \mathbb{L}_{1}^{-1}\left(\sigma_{1}\right)\right) \backslash\left(\bigcup_{i=1}^{N_{1}} \mathcal{R}_{i}\right) .
$$

Note that each $\mathcal{D}_{i}$ forms a polygon. As before for simplicity we will assume none of the sides of $\partial \Omega_{\epsilon^{-\frac{1}{2}}}$ is parallel to $w_{1}$. Let $c_{\Omega}$ denote the length of the shortest side of $\partial \Omega$, we can assume without loss of generality $\sqrt{\epsilon}<c_{\Omega}$, so we have that any $\overline{\mathcal{D}_{i}}$ will intersect at most two sides of $\partial \Omega_{\epsilon^{-\frac{1}{2}}}$. Let $E_{1}:=\left\{i \in\left\{1,2, \ldots, N_{2}\right\}: \partial \mathcal{D}_{i}\right.$ has 4 sides $\}$. So any $i \in\left\{1,2, \ldots, N_{2}\right\} \backslash E_{1}$ is such that $\partial \mathcal{D}_{i}$ has 5 or 3 sides.

Let $E_{2}:=\left\{i \in\left\{1,2, \ldots, N_{2}\right\}: \partial \mathcal{D}_{i}\right.$ has 5 sides $\}$. For any $i \in E_{2}$ let $a_{i}, b_{i}$ be the endpoints of $\partial \Omega_{\epsilon^{-\frac{1}{2}}} \cap \overline{\mathcal{D}_{i}}$ and let $c_{i}, d_{i}$ denote the corners of the polytope $\mathcal{D}_{i}$ that do not intersect $\partial \Omega_{\epsilon^{-\frac{1}{2}}}$.

Define $\widetilde{\mathcal{D}_{i}}=\operatorname{conv}\left(a_{i}, b_{i}, c_{i}, d_{i}\right)$ for $i \in E_{2}$ and define $\widetilde{\mathcal{D}_{i}}=\mathcal{D}_{i}$ for $i \in E_{1}$. Finally define $T_{i}:=\mathcal{D}_{i} \backslash \widetilde{\mathcal{D}_{i}}$ for $i \in E_{2}$, note each $T_{i}$ forms a triangle.

For each $i \in E_{1} \cup E_{2}$ we can split each $\widetilde{\mathcal{D}_{i}}$ into two triangles $\tau_{i}^{1}, \tau_{i}^{2}$, each of which has a side parallel to $w_{1}$ (i.e. $\overline{\widetilde{\mathcal{D}}_{i}}=\overline{\tau_{i}^{1} \cup \tau_{i}^{2}}$ ). Let $\left\{\tau_{2 N_{1}+1}, \tau_{2 N_{1}+2}, \ldots, \tau_{N_{3}}\right\}$ denote the additional set of triangles that are formed by

$$
\left\{\tau_{i}^{q}: i \in E_{1} \cup E_{2}, q \in\{1,2\}\right\},\left\{\mathcal{D}_{i}: i \in\left\{1,2, \ldots, N_{2}\right\} \backslash\left(E_{1} \cup E_{2}\right)\right\} \text { and }\left\{T_{i}: i \in E_{2}\right\}
$$

and let

$$
\mathbb{B}_{d}:=\left\{i \in\left\{1,2, \ldots, N_{3}\right\}: \tau_{i} \subset N_{64 \sigma^{-2}}\left(\partial \Omega_{\epsilon^{-\frac{1}{2}}}\right)\right\}
$$

Firstly we will show that

$$
N_{3}-2 N_{1} \leq c \epsilon^{-\frac{1}{2}} \text { and } \operatorname{Card}\left(\mathbb{B}_{d}\right) \leq c \epsilon^{-\frac{1}{2}}
$$

Secondly let $l_{i}$ be the affine interpolation of $v$ on the corners of $\tau_{i}$ for $i \in \mathbb{B}_{d}$ we will also show

$$
\sum_{i \in \mathbb{B}_{d}}\left|D l_{i}\right|^{2} \leq c \epsilon^{-1} m_{\epsilon}^{p}
$$

Proof of Step 5. To start with since $\bigcup_{i \in \mathbb{B}_{d}} \tau_{i} \subset N_{64 \sigma^{-2}}\left(\partial \Omega_{\epsilon^{-\frac{1}{2}}}\right)$ and since $L^{2}\left(\tau_{i}\right)>c$ for any $i \in \mathbb{B}_{d}$. So

$$
\begin{aligned}
\operatorname{Card}\left(\mathbb{B}_{d}\right) & \leq c L^{2}\left(N_{64 \sigma^{-2}}\left(\partial \Omega_{\epsilon^{-\frac{1}{2}}}\right)\right) \\
& \leq c \epsilon^{-\frac{1}{2}}
\end{aligned}
$$

note also $\left\{2 N_{1}+1, \ldots, N_{3}\right\} \subset \mathbb{B}_{d}$ which gives (5.96).

For any $i \in E_{1} \cup E_{2}$ we will order the triangles $\tau_{i}^{1}, \tau_{i}^{2}$ so that two of the corners of $\tau_{i}^{2}$ intersects $\partial \Omega_{\epsilon^{-\frac{1}{2}}}$ and two of the corners of $\tau_{i}^{1}$ intersects $\bigcup_{i \in\left\{1,2, \ldots, 2 N_{1}\right\}} \overline{\mathcal{R}_{i}}$.

So let $\left\{a_{i}, b_{i}, c_{i}\right\}$ denote the corners of $\tau_{i}^{1}$ we can order them so that $\frac{a_{i}-b_{i}}{\left|a_{i}-b_{i}\right|}=w_{1}$ and $\frac{c_{i}-b_{i}}{\left|c_{i}-b_{i}\right|}=w_{2}$. So $\left[a_{i}, b_{i}\right] \subset \mathbb{L}_{1}^{-1}\left(\sigma_{1}\right),\left[c_{i}, b_{i}\right] \subset \mathbb{L}_{2}^{-1}\left(\sigma_{2}\right)$. So by definition of $\mathbb{L}_{1}^{-1}\left(\sigma_{1}\right)$ we have that

$$
\left[a_{i}, b_{i}\right] \subset\left(\mathbb{R}_{+} w_{1}+\left(t+k_{1}\right) w_{2}\right) \cup\left(\mathbb{R}_{-} w_{1}+\left(t+k_{1}\right) w_{2}\right)
$$

for some $k_{1} \in\left\{Q_{1}^{1}, Q_{1}^{1}+1, \ldots, Q_{2}^{1}-1\right\}, \sigma_{1} \in \mathrm{P}_{1}^{+} \cap \mathrm{P}_{1}^{-}$. By definition (5.43) and by (5.42) we have that $\left[a_{i}, b_{i}\right] \cap \mathrm{A}_{0} \neq \emptyset$. So there exists $x_{i} \in\left[a_{i}, b_{i}\right]$ such that $d\left(D v\left(x_{i}\right), K\right) \leq 1$. Thus

$$
\sup \left\{|D v(z)|: z \in\left[a_{i}, b_{i}\right] \cup\left[b_{i} \cdot c_{i}\right]\right\} \leq c+\int_{\left[a_{i}, b_{i}\right] \cup\left[b_{i}, c_{i}\right]}\left|D^{2} v(z)\right| \mathrm{d} H^{1} z .
$$


Let $L_{i}^{1}$ be the affine function we obtain from the interpolation of $v$ on the corners of $\tau_{i}^{1}$. We have

$$
\begin{aligned}
\left|D L_{i}^{1} w_{1}\right| & \leq c\left|v\left(a_{i}\right)-v\left(b_{i}\right)\right| \\
& \leq c \int_{\left[a_{i}, b_{i}\right]}|D v(z)| \mathrm{d} H^{1} z \\
\stackrel{(5.98)}{\leq} & c+c \int_{\left[a_{i}, b_{i}\right] \cup\left[b_{i}, c_{i}\right]}\left|D^{2} v(z)\right| \mathrm{d} H^{1} z .
\end{aligned}
$$

And in exactly the same way we have

$$
\left|D L_{i}^{1} w_{2}\right|=\left|\frac{L_{i}^{1}\left(c_{i}\right)-L_{i}^{1}\left(b_{i}\right)}{\left|c_{i}-b_{i}\right|}\right| \leq+c \int_{\left[a_{i}, b_{i}\right] \cup\left[b_{i}, c_{i}\right]}\left|D^{2} v(z)\right| \mathrm{d} H^{1} z .
$$

Thus

$$
\begin{aligned}
\left|D L_{i}^{1}\right|^{2} & =c\left(\left|D L_{i}^{1} w_{1}\right|^{2}+\left|D L_{i}^{1} w_{2}\right|^{2}\right) \\
& \leq c+c\left(\int_{\left[a_{i}, b_{i}\right] \cup\left[b_{i}, c_{i}\right]}\left|D^{2} v(z)\right| \mathrm{d} H^{1} z\right)^{2} \\
& \leq c+c \int_{\partial \tau_{i}^{1} \cap\left(\mathbb{L}_{1}^{-1}\left(\sigma_{1}\right) \cup \mathbb{L}_{2}^{-1}\left(\sigma_{2}\right)\right)}\left|D^{2} v(z)\right|^{2} \mathrm{~d} H^{1} z .
\end{aligned}
$$

Now let us consider the triangle $\tau_{i}^{2}$. Let $\left\{a_{i}, b_{i}, c_{i}\right\}$ denote the corners of $\tau_{i}^{2}$ where we have ordered $a_{i}, b_{i}, c_{i}$ such that $\frac{a_{i}-b_{i}}{\left|a_{i}-b_{i}\right|}=w_{1}$ and $b_{i}, c_{i} \in \partial \Omega_{\epsilon^{-\frac{1}{2}}}$. Let $L_{i}^{2}$ denote the affine map we get from interpolation of $v$ on the corners of $\tau_{i}^{2}$. Arguing exactly as we have before we can show that

$$
\left|D L_{i}^{2} w_{1}\right|^{2} \leq c+c \int_{\partial \tau_{i}^{2} \cap\left(\mathbb{L}_{1}^{-1}\left(\sigma_{1}\right) \cup\left(\mathbb{L}_{2}^{-1}\left(\sigma_{2}\right)\right)\right.}\left|D^{2} v(z)\right|^{2} \mathrm{~d} H^{1} z .
$$

Now $\left|D L_{i}^{2}\left(\frac{b_{i}-c_{i}}{\left|b_{i}-c_{i}\right|}\right)\right|^{2} \leq c\left|l_{F}\left(b_{i}\right)-l_{F}\left(c_{i}\right)\right|^{2} \leq c$. Since $w_{1}$ and $\frac{b_{i}-c_{i}}{\left|b_{i}-c_{i}\right|}$ are not parallel this implies

$$
\left|D L_{i}^{2}\right|^{2} \leq c+c \int_{\partial \tau_{i}^{2} \cap\left(\mathbb{L}_{1}^{-1}\left(\sigma_{1}\right) \cup \mathbb{L}_{2}^{-1}\left(\sigma_{2}\right)\right)}\left|D^{2} v(z)\right|^{2} \mathrm{~d} H^{1} z .
$$

Now for any $i \in\left\{1,2, \ldots, N_{2}\right\} \backslash\left(E_{1} \cup E_{2}\right), \mathcal{D}_{i}$ forms a triangle with the corners in $\partial \Omega_{\epsilon^{-\frac{1}{2}}}$, let $I_{i}$ be the affine map we obtain by interpolation of $v$ on the corners of $\mathcal{D}_{i}$, then $I_{i}$ has the property that

$$
\left|D I_{i}\right| \leq c \text { for any } i \in\left\{1,2, \ldots, N_{2}\right\} \backslash\left(E_{1} \cup E_{2}\right) .
$$

For any $i \in E_{2}$ let $J_{i}$ be the affine function we get from interpolating $v$ on $T_{i}$, since again the corners of $\tau_{i}$ belong to $\partial \Omega_{\epsilon^{-\frac{1}{2}}}$ we have

$$
\left|D J_{i}\right| \leq c \text { for any } i \in E_{2} .
$$

Let $l_{i}$ be the affine map we obtain from interpolating $v$ on $\tau_{i}$ for $i \in \mathbb{B}_{d}$. For any $i \in \mathbb{B}_{d} \backslash\left\{2 N_{1}+1, \ldots, N_{3}\right\}$ let $\left\{a_{i}, b_{i}, c_{i}\right\}$ denote the corners of $\tau_{i}$ where $\frac{a_{i}-b_{i}}{\left|a_{i}-b_{i}\right|}=w_{1}$ and $\frac{c_{i}-b_{i}}{\left|c_{i}-b_{i}\right|}=w_{2}$. Exactly as in the case where we considered triangle $\tau_{i}^{1}$ for $i \in E_{1} \cup E_{2}$ we must have that $\left[a_{i}, b_{i}\right] \subset \mathbb{L}_{1}^{-1}\left(\sigma_{1}\right)$ and $\left[c_{i}, b_{i}\right] \subset \mathbb{L}_{2}^{-1}\left(\sigma_{2}\right)$. We will assume $a_{i}, b_{i}$ are ordered so that $\mathrm{d}\left(a_{i}, \partial \Omega_{\epsilon^{-\frac{1}{2}}}\right)<d\left(b_{i}, \partial \Omega_{\epsilon^{-\frac{1}{2}}}\right)$. Let $d_{i} \in \partial \Omega_{\epsilon^{-\frac{1}{2}}}$ be such that $\left[a_{i}, b_{i}\right] \subset\left[d_{i}, b_{i}\right]$. 
By definition of $\mathbb{B}_{d}$ we know $\left|d_{i}-b_{i}\right|<32 \sigma^{-2}$. Let $\Gamma_{i}:=\left[d_{i}, b_{i}\right] \cup\left[b_{i}, c_{i}\right]$, by arguing exactly the same way as we did to show (5.99) we have

$$
\left|D l_{i}\right|^{2} \leq c+\int_{\Gamma_{i}}\left|D^{2} v(z)\right|^{2} \mathrm{~d} H^{1} z .
$$

So let $l_{i}$ be the affine map we obtain from interpolating $v$ on $\tau_{i}$ for $i \in \mathbb{B}_{d}$ we have by (5.99), (5.100), (5.101), (5.102) and (5.103)

$$
\begin{array}{cc}
\sum_{i \in \mathbb{B}_{d}}\left|D l_{i}\right|^{2} \stackrel{(5.96)}{\leq} c \epsilon^{-\frac{1}{2}}+c \int_{\mathbb{L}_{1}^{-1}\left(\sigma_{1}\right) \cup \mathbb{U}_{2}^{-1}\left(\sigma_{2}\right)}\left|D^{2} v(z)\right|^{2} \mathrm{~d} H^{1} z \\
\stackrel{(4.2),(5.59)}{\leq} c \epsilon^{-1} m_{\epsilon}^{p} .
\end{array}
$$

Step 6. Let $w \in \mathcal{F}_{F}^{\sqrt{\epsilon}, \varsigma}$ be defined by $w(z)=l_{i}(z)$ for $z \in \tau_{i}, i=1,2, \ldots, N_{3}$. We will show that

$$
\sum_{i \in J(w)} \sum_{M \in N_{i}(w)}\left|D w_{\left\lfloor\tau_{i}\right.}-M\right|^{2} \leq c \epsilon^{-1} m_{\epsilon}^{p}
$$

Proof of Step 6. Let

$$
V_{1}(i)=\left\{j \in\left\{1,2, \ldots, N_{3}\right\}: H^{1}\left(\overline{\tau_{i}} \cap \overline{\tau_{j}}\right)>0\right\}
$$

Let

$$
\mathbb{I}_{0}:=\left\{i \in\left\{1,2, \ldots, N_{3}\right\}: \tau_{i} \subset \Omega \backslash N_{32 \sigma^{-2}}(\partial \Omega)\right\} .
$$

Note that for any $i \in\left\{1,2, \ldots, N_{3}\right\} \backslash \mathbb{I}_{0}, V_{1}(i) \subset \mathbb{B}_{d}$. So

$$
\begin{aligned}
\sum_{J(w) \backslash \mathbb{I}_{0}} \sum_{M \in N_{i}(w)}\left|D w_{\left\lfloor\tau_{i}\right.}-M\right|^{2} & \leq \sum_{i \in J(w) \backslash \mathbb{I}_{0}}\left(\sum_{j \in V_{1}(i)}\left|D l_{i}-D l_{j}\right|^{2}+\left|D l_{i}-F\right|^{2}\right) \\
& \leq \quad c \sum_{i \in \mathbb{B}_{d}}\left|D l_{i}\right|^{2}+c \operatorname{Card}\left(\mathbb{B}_{d}\right) \\
\stackrel{(5.96),(5.97),(4.2)}{\leq} & c \epsilon^{-1} m_{\epsilon}^{p} .
\end{aligned}
$$

Also note that if $i \in \mathbb{I}_{0}$ then $V_{1}(i) \subset\left\{1,2, \ldots, 2 N_{1}\right\}$ and $V_{1}(i)=V_{0}(i)$ (see definition (5.84)) in addition we know $\partial \tau_{i} \cap \partial \Omega=\emptyset$ so $N_{i}(w)=V_{0}(i)$ and $J(w) \cap \mathbb{I}_{0}=\Upsilon_{0}$ (see (5.85)). So

$$
\begin{aligned}
& \sum_{i \in J(w) \cap \mathbb{I}_{0}} \sum_{M \in N_{i}(w)}\left|D w_{\left\lfloor\tau_{i}\right.}-M\right|^{2}=\sum_{i \in \Upsilon_{0}} \sum_{j \in V_{0}(i)}\left|D l_{i}-D l_{j}\right|^{2} \\
& \stackrel{(5.86)}{\leq} c \epsilon^{-1} m_{\epsilon}^{p}
\end{aligned}
$$

Now

$$
\begin{aligned}
\sum_{i \in J(w)} \sum_{M \in N_{i}(w)}\left|D w_{\left\lfloor\tau_{i}\right.}-M\right|^{2}= & \sum_{i \in J(w) \cap \mathbb{I}_{0}} \sum_{M \in N_{i}(w)}\left|D w_{\left\lfloor\tau_{i}\right.}-M\right|^{2} \\
& +\sum_{i \in J(w) \backslash \mathbb{I}_{0}} \sum_{M \in N_{i}(w)}\left|D w_{\left\lfloor\tau_{i}\right.}-M\right|^{2} \\
\stackrel{(5.108),(5.109)}{\leq} & c \epsilon^{-1} m_{\epsilon}^{p} .
\end{aligned}
$$


Step 7. We will show

$$
\sum_{j=1}^{N_{3}} d^{p}\left(D w_{\left\lfloor\tau_{i}\right.}, K\right) \leq c \epsilon^{-1} m_{\epsilon}^{p} .
$$

Proof of Step 7. Since for any $j \in\left\{2 N_{1}+1, \ldots, N_{3}\right\}$ we have

$$
d^{p}\left(D w_{\left\lfloor\tau_{j}\right.}, K\right) \leq c+\left|D w_{\left\lfloor\tau_{j} \mid\right.}\right|^{p} \leq c+\left|D w_{\left\lfloor\tau_{j}\right.}\right|^{2}
$$

so using the fact $\left\{2 N_{1}+1, \ldots, N_{3}\right\} \subset \mathbb{B}_{d}$ for the last inequality

$$
\begin{array}{cl}
\sum_{j=1}^{N_{3}} d^{p}\left(D w_{\left\lfloor\tau_{i}\right.}, K\right) & \stackrel{(5.82),(5.111)}{\leq} \\
\qquad \epsilon^{-1} m_{\epsilon}^{p}+c\left(N_{3}-2 N_{1}+1\right)+\sum_{j=2 N_{1}+1}^{N_{3}}\left|D w_{\left\lfloor\tau_{j}\right.}\right|^{2} \\
\stackrel{(4.2),(5.96),(5.97)}{\leq} & c \epsilon^{-1} m_{\epsilon}^{p} .
\end{array}
$$

Step 8. We will show that (for small enough $\varsigma$ ) there exists a function $\tilde{u} \in \mathcal{D}_{F}^{\varsigma, h}$ such that

$$
\int_{\Omega} d^{p}(D \tilde{u}(z), K) \mathrm{d} L^{2} z \leq c m_{\epsilon}^{p}
$$

Proof of Step 8. Recall definition of $d_{0}$, see (4.3). Let

$$
\mathrm{G}_{g}:=\left\{i \in\left\{1,2, \ldots, N_{3}\right\}: d\left(D w_{\left\lfloor\tau_{i}\right.}, K\right) \leq d_{0}\right\}
$$

Recall $V_{1}(i)$ is defined by (5.106). Let $\mathbb{V}(i):=\bigcup_{k \in V_{1}(i)} V_{1}(k)$ and (recall the definition of $\mathbb{I}_{0}$, see $\left.(5.107)\right)$ let $\mathrm{G}_{g i}:=\left\{i \in \mathbb{I}_{0}: \mathbb{V}(i) \subset \mathrm{G}_{g}\right\}$. Note $\operatorname{Card}(\mathbb{V}(i)) \leq 12$. Let $\mathbb{A}_{0}:=\bigcup_{i \in \mathbb{I}_{0} \backslash \mathrm{G}_{g i}} \overline{\tau_{i}}$, so

$$
L^{2}\left(\mathbb{A}_{0}\right) \geq c \operatorname{Card}\left(\mathbb{I}_{0} \backslash \mathrm{G}_{g i}\right)
$$

Let $\mathrm{O}_{i}:=\bigcup_{j \in \mathbb{V}(i)} \overline{\tau_{j}}$, to by applying the $5 r$ Covering Theorem (see Thm. 2.1 [25]) we can find a subset $\left\{i_{1}, i_{2}, \ldots, i_{P_{1}}\right\} \subset \mathbb{I}_{0} \backslash \mathrm{G}_{g i}$ such that

$$
\mathbb{A}_{0} \subset \bigcup_{k=1}^{P_{1}} N_{60}\left(\mathrm{O}_{i_{k}}\right)
$$

and $\left\{\mathrm{O}_{i_{1}}, \mathrm{O}_{i_{2}}, \ldots, \mathrm{O}_{i_{P_{1}}}\right\}$ are disjoint. Note (5.113), (5.114) imply $P_{1} \geq c$ Card $\left(\mathbb{I}_{0} \backslash \mathrm{G}_{g i}\right)$ and since for every $k \in\left\{1,2, \ldots, P_{1}\right\}$ since $\mathbb{V}\left(i_{k}\right) \not \subset \mathrm{G}_{g i}$ (by definition of $\mathrm{G}_{g i}$ ) we can find $q_{k} \in\left\{1,2, \ldots, N_{3}\right\}$ such that $\tau_{q_{k}} \subset \mathrm{O}_{i_{k}}$ and $d\left(D w_{\left\lfloor\tau_{q_{k}}\right.}, K\right)>d_{0}$. We also know that $\left\{\tau_{q_{1}}, \tau_{q_{2}}, \ldots, \tau_{q_{P_{1}}}\right\}$ are disjoint. So

$$
d_{0}^{p} P_{1} \leq \sum_{k=1}^{P_{1}} d^{p}\left(D w_{\left\lfloor\tau_{q_{k}}\right.}, K\right) \stackrel{(5.110)}{\leq} c \epsilon^{-1} m_{\epsilon}^{p}
$$

Thus Card $\left(\mathbb{I}_{0} \backslash \mathrm{G}_{g i}\right) \leq c \epsilon^{-1} m_{\epsilon}^{p} \stackrel{(5.33)}{\leq} c \mathcal{C}_{0} \epsilon^{-1}$. Now Card $\left(\mathbb{I}_{0}\right) \geq c \epsilon^{-1}$ so

$$
\operatorname{Card}\left(\mathbb{I}_{0} \cap \mathrm{G}_{g i}\right) \geq c \epsilon^{-1}-c \mathcal{C}_{0} \epsilon^{-1} .
$$

Assuming constant $\mathcal{C}_{0}$ at the start of Proposition 5.2 was chosen small enough we have

$$
\operatorname{Card}\left(\mathbb{I}_{0} \cap \mathrm{G}_{g i}\right) \geq c \epsilon^{-1} .
$$


Note that again by applying the $5 r$ covering Theorem we can find subset $\left\{j_{1}, j_{2}, \ldots, j_{P_{2}}\right\} \subset \mathbb{I}_{0} \cap \mathrm{G}_{g i}$ such that

$$
\bigcup_{i \in \mathbb{I}_{0} \cap G_{g i}} \tau_{i} \subset \bigcup_{k=1}^{P_{2}} N_{60}\left(\mathrm{O}_{j_{k}}\right)
$$

and $\left\{\mathrm{O}_{j_{1}}, \mathrm{O}_{j_{2}}, \ldots, \mathrm{O}_{j_{P_{2}}}\right\}$ are disjoint. Inequalities (5.115) and (5.116) imply that

$$
P_{2} \geq c \epsilon^{-1} \text {. }
$$

We denote the corners of $\tau_{i}$ by $\left\{\omega_{i}^{1}, \omega_{i}^{2}, \omega_{i}^{3}\right\}$ for any $i=1,2, \ldots, N_{3}$. Let $q \in\left\{1,2, \ldots, P_{2}\right\}$ and pick $c_{q} \in$ $\left\{\omega_{j_{q}}^{1}, \omega_{j_{q}}^{2}, \omega_{j_{q}}^{3}\right\}$. Let $\mathbb{W}\left(j_{q}\right) \subset \mathbb{V}\left(j_{q}\right)$ be defined by $\mathbb{W}\left(j_{q}\right):=\left\{k \in \mathbb{V}\left(j_{q}\right): \overline{\tau_{k}} \cap c_{q} \neq \emptyset\right\}$. Note that for any $k \in$ $\mathbb{W}\left(j_{q}\right)$, since $\mathbb{V}\left(j_{q}\right) \subset \mathrm{G}_{g}$ we have

$$
\left|w\left(\omega_{k}^{a}\right)-w\left(c_{q}\right)\right| \leq 4 \sigma^{-1} \text { for any } a \in\{1,2,3\} .
$$

For each $k \in \mathbb{W}\left(j_{q}\right)$ define the affine map $\tilde{l}_{k}: \tau_{k} \rightarrow \mathbb{R}^{2}$ by

$$
\tilde{l}_{k}(b)= \begin{cases}w(b) & \text { for } b \in\left\{\omega_{k}^{1}, \omega_{k}^{2}, \omega_{k}^{3}\right\} \backslash\left\{c_{q}\right\} \\ w\left(c_{q}\right)+30 \sigma^{-1} e_{1} & \text { for } b=c_{q} .\end{cases}
$$

For simplicity we order the corners $\left\{\omega_{k}^{1}, \omega_{k}^{2}, \omega_{k}^{3}\right\}$ so that $\omega_{k}^{1}=c_{q}$. Note

$$
\begin{aligned}
& \left|D \tilde{l}_{k}\left(\frac{\omega_{k}^{1}-\omega_{k}^{2}}{\left|\omega_{k}^{1}-\omega_{k}^{2}\right|}\right)\right|=\left|\omega_{k}^{1}-\omega_{k}^{2}\right|^{-1}\left|w\left(\omega_{k}^{1}\right)-w\left(\omega_{k}^{2}\right)+30 \sigma^{-1} e_{1}\right| \\
& \stackrel{(5.118)}{\geq} 10 \sigma^{-1}
\end{aligned}
$$

In exactly the same way we have $\left|D \tilde{l}_{k}\left(\frac{\omega_{k}^{1}-\omega_{k}^{3}}{\left|\omega_{k}^{1}-\omega_{k}^{3}\right|}\right)\right| \geq 10 \sigma^{-1}$ which implies

$$
\left|D \tilde{l}_{k}\right| \geq 10 \sigma^{-1}
$$

In a very similar way we can show $\left|D \tilde{l}_{k}\left(\frac{\omega_{k}^{1}-\omega_{k}^{2}}{\mid \omega_{k}^{1}-\omega_{k}^{2}}\right)\right| \leq 60 \sigma^{-1},\left|D \tilde{l}_{k}\left(\frac{\omega_{k}^{1}-\omega_{k}^{3}}{\left|\omega_{k}^{1}-\omega_{k}^{3}\right|}\right)\right| \leq 60 \sigma^{-1}$ and thus

$$
\left|D \tilde{l}_{k}\right| \leq 60 \sigma^{-1} \text {. }
$$

From (5.119) we know

$$
\begin{aligned}
\sum_{k \in \mathbb{W}\left(j_{q}\right)} d^{p}\left(D \tilde{l}_{k}, K\right) L^{2}\left(\tau_{k}\right) & \geq d^{p}\left(D \tilde{l}_{j_{q}}, K\right) L^{2}\left(\tau_{j_{q}}\right) \\
& \stackrel{(5.119)}{\geq} 9 \sigma^{-p} L^{2}\left(\tau_{j_{q}}\right)
\end{aligned}
$$

and

$$
\sum_{k \in \mathbb{W}\left(j_{q}\right)} d^{p}\left(D \tilde{l}_{k}, K\right) L^{2}\left(\tau_{k}\right) \stackrel{(5.120)}{\leq} 120^{2} \sigma^{-2} \times 100 \varsigma^{-2}
$$


Note recall from (5.117) $P_{2} \geq c \epsilon^{-1} \stackrel{(5.33)}{>} \frac{m_{\epsilon}^{p}}{\epsilon}$ so we can define piecewise affine function $\tilde{v}: \Omega_{\epsilon^{-\frac{1}{2}}} \rightarrow \mathbb{R}^{2}$ by

$$
\tilde{v}(z)= \begin{cases}w(z) & \text { for } z \in \tau_{i}, i \in\left\{1,2, \ldots, N_{3}\right\} \backslash\left(\bigcup_{q=1}^{\left[\epsilon^{-1} m_{\epsilon}^{p}\right]} \mathbb{W}\left(j_{q}\right)\right) \\ \tilde{l}_{i}(z) & \text { for } z \in \tau_{i}, i \in\left(\bigcup_{q=1}^{\left[\epsilon^{-1} m_{\epsilon}^{p}\right]} \mathbb{W}\left(j_{q}\right)\right) .\end{cases}
$$

So

$$
\begin{aligned}
& \int_{\Omega_{\epsilon^{-\frac{1}{2}}}} d^{p}(D \tilde{v}(z), K) \mathrm{d} L^{2} z \quad=\quad \sum_{i \in\left\{1,2, \ldots, N_{3}\right\} \backslash\left(\cup_{q=1}^{\left[\epsilon^{-1} m_{\epsilon}^{p}\right]} \mathbb{W}\left(j_{q}\right)\right)} d^{p}\left(D w_{\left\lfloor\tau_{i}\right.}, K\right) L^{2}\left(\tau_{i}\right) \\
& +\sum_{i \in\left(\cup_{q=1}^{\left[\epsilon^{-1} m_{\epsilon}^{p}\right]} \mathbb{W}\left(j_{q}\right)\right)} d^{p}\left(D \tilde{l}_{i}, K\right) L^{2}\left(\tau_{i}\right) \\
& \stackrel{(5.110),(5.122)}{\leq} c \epsilon^{-1} m_{\epsilon}^{p}+c\left[\epsilon^{-1} m_{\epsilon}^{p}\right] \\
& \leq \quad c \epsilon^{-1} m_{\epsilon}^{p}
\end{aligned}
$$

and

$$
\begin{aligned}
& \int_{\Omega_{\epsilon^{-\frac{1}{2}}}} d^{p}(D \tilde{v}(z), K) \mathrm{d} L^{2} z \geq \sum_{q=1}^{\left[\epsilon^{-1} m_{\epsilon}^{p}\right]} \int_{\mathrm{O}_{j_{q}}} d^{p}(D \tilde{v}(z), K) \mathrm{d} L^{2} z \\
& \stackrel{(5.121)}{\geq} c\left[\epsilon^{-1} m_{\epsilon}^{p}\right] .
\end{aligned}
$$

Let $\mathbb{Y}:=\left\{i \in\left\{1,2, \ldots, N_{3}\right\}: V_{1}(i) \cap\left(\bigcup_{q=1}^{\left[\epsilon^{-1} m_{\epsilon}^{p}\right]} \mathbb{W}\left(j_{q}\right)\right)=\emptyset\right\}$. Note

$$
\operatorname{Card}\left(\left\{1,2, \ldots, N_{3}\right\} \backslash \mathbb{Y}\right) \leq c \epsilon^{-1} m_{\epsilon}^{p}
$$

and note

$$
\sum_{M \in N_{i}(\tilde{v})}\left|D \tilde{v}_{\left\lfloor\tau_{i}\right.}-M\right|^{2} \leq \sum_{M \in N_{i}(w)}\left|D w_{\left\lfloor\tau_{i}\right.}-M\right|^{2}+c \text { for any } i \in J(\tilde{v}) \backslash \mathbb{Y}
$$

so as $J(\tilde{v}) \cap \mathbb{Y}=J(w) \cap \mathbb{Y}$ and $D \tilde{v}_{\left\lfloor\tau_{j}\right.}=D w_{\left\lfloor\tau_{j}\right.}$ for every $j \in \bigcup_{i \in J(\tilde{v}) \cap \mathbb{Y}} V_{1}(i)$ we have

$$
\begin{array}{cl}
\sum_{i \in J(\tilde{v})} \sum_{N_{i}(\tilde{v})}\left|D \tilde{v}_{\left\lfloor\tau_{i}\right.}-M\right|^{2}= & \sum_{i \in J(w) \cap \mathbb{Y}} \sum_{M \in N_{i}(w)}\left|D w_{\left\lfloor\tau_{i}\right.}-M\right|^{2} \\
& +\sum_{i \in J(\tilde{v}) \backslash \mathbb{Y}} \sum_{M \in N_{i}(\tilde{v})}\left|D \tilde{v}_{\left\lfloor\tau_{i}\right.}-M\right|^{2} \\
& c \epsilon^{-1} m_{\epsilon}^{p}+c \operatorname{Card}(J(\tilde{v}) \backslash \mathbb{Y}) \\
(5.105),(5.106) & \leq \epsilon^{-1} m_{\epsilon}^{p} .
\end{array}
$$

Thus

$$
\int_{\Omega_{\epsilon^{-\frac{1}{2}}}} d^{p}(D \tilde{v}(z), K) \mathrm{d} L^{2} z \stackrel{(5.124),(5.127)}{\geq} c \sum_{i \in J(\tilde{v})} \sum_{M \in N_{i}(\tilde{v})}\left|D \tilde{v}_{\left\lfloor\tau_{i}\right.}-M\right|^{2}
$$


Define $\tilde{u}(z)=\tilde{v}(\sqrt{\epsilon} z) \epsilon^{-\frac{1}{2}}$. We have that

$$
\int_{\Omega} d^{p}(D \tilde{u}(z), K) \mathrm{d} L^{2} z=\epsilon \int_{\Omega_{\epsilon}-\frac{1}{2}} d^{p}(D \tilde{v}(z), K) \mathrm{d} L^{2} z
$$

and thus

$$
\int_{\Omega} d^{p}(D \tilde{u}(z), K) \mathrm{d} L^{2} z \stackrel{(5.128)}{\geq} c \epsilon \sum_{i \in J(\tilde{v})} \sum_{M \in N_{i}(\tilde{v})}\left|D \tilde{v}_{\left\lfloor\tau_{i}\right.}-M\right|^{2} .
$$

Now (for small enough $\varsigma$ ) $\left\{\sqrt{\epsilon} \tau_{i}\right\}$ forms a $(h, \varsigma)$ triangulation of $\Omega$ and it is easy to see that

$$
\sum_{i \in J(\tilde{u})} \sum_{M \in N_{i}(\tilde{u})}\left|D \tilde{u}_{\left\lfloor\sqrt{\epsilon} \tau_{i}\right.}-M\right|^{2}=\sum_{i \in J(\tilde{v})} \sum_{M \in N_{i}(\tilde{v})}\left|D \tilde{v}_{\left\lfloor\tau_{i}\right.}-M\right|^{2} .
$$

Thus (again assuming $\varsigma$ is small enough) we have from (5.130)

$$
\sum_{i \in J(\tilde{u})} \sum_{M \in N_{i}(\tilde{u})} \epsilon\left|D \tilde{u}_{\left\lfloor\sqrt{\epsilon} \tau_{i}\right.}-M\right|^{2} \leq \frac{\varsigma^{-1}}{2} \int_{\Omega} d^{p}(D \tilde{u}(z), K) \mathrm{d} L^{2} z .
$$

Thus we have that $u \in \mathcal{D}_{F}^{\varsigma, \sqrt{\epsilon}}$. We also know from (5.129) and (5.123) that $\tilde{u}$ satisfies (5.112).

Proposition 5.3. Let $w_{1} \in S^{1}$ be such that $w_{2} \in w_{1}^{\perp}$ we have that $w_{1}, w_{2}$ and $\frac{w_{1}-w_{2}}{\left|w_{1}-w_{2}\right|}$ are not in the set of rank-1 connections between $S O(2) A_{i}$ and $S O(2) A_{j}$ for any $i \neq j$. Let $p \in[1,2]$. Let $F \notin K$, given function $u \in \mathcal{D}_{F}^{\varsigma, \sqrt{\epsilon}}$ we define $w: \Omega_{2} \rightarrow \mathbb{R}^{2}$ by

$$
\tilde{w}(z)= \begin{cases}u(z) & \text { if } z \in \Omega \\ l_{F}(z) & \text { if } z \in \Omega_{2} \backslash \Omega\end{cases}
$$

We will show there exists a small positive constant $\eta=\eta\left(w_{1}, A_{1}, \ldots, A_{N}\right)$ such that for $\tilde{w}=w * \rho_{\eta \sqrt{\epsilon}}$ and

$$
w(z)=\tilde{w}\left(\frac{z}{1+\eta \sqrt{\epsilon}}\right)(1+\eta \sqrt{\epsilon})
$$

then $w \in A_{F}$ and $w$ satisfies

$$
\int_{\Omega} d^{p}(D w(z), K)+\epsilon\left|D^{2} w(z)\right|^{2} \mathrm{~d} L^{2} z \leq c \int_{\Omega} d^{p}(D u(z), K) \mathrm{d} L^{2} z .
$$

Proof. Firstly note $u$ is piecewise affine on a triangulation which we will label $\left\{\tau_{1}, \tau_{2}, \ldots, \tau_{N_{3}}\right\}$. Given triangle $\tau_{i}$ we define the neighbouring gradients $N_{i}(u)$ by (1.3) and we define the jump triangles $J_{i}(u)$ by $(1.4)$. Now since $u \in \mathcal{D}_{F}^{\varsigma, \sqrt{\epsilon}}$ we have

$$
\sum_{i \in J(u)} \sum_{M \in N_{i}(u)}\left|D u_{\left\lfloor\tau_{i}\right.}-M\right|^{2} \leq \varsigma^{-1} \epsilon^{-1} \int_{\Omega} d^{p}(D u(z), K) \mathrm{d} L^{2} z .
$$

Let $v(z)=u(\sqrt{\epsilon} z) \epsilon^{-\frac{1}{2}}$. Let

$$
\alpha_{0}=\int_{\Omega_{\epsilon-\frac{1}{2}}} d^{p}(D v(z), K) \mathrm{d} L^{2} z .
$$

Let $V(j):=\left\{k: H^{1}\left(\overline{\tau_{k}} \cap \overline{\tau_{j}}\right)>0\right\}$. Define $\mathbb{V}_{0}(i):=\bigcup_{j \in V(i)} V(j)$ and $\mathbb{V}_{1}(i):=\bigcup_{j \in \mathbb{V}_{0}(i)} V(j)$. 
Let $G_{0}:=\left\{i: \mathrm{d}\left(D v_{\left\lfloor\tau_{i}\right.}, K\right) \leq \eta\right\}$. Let $\mathcal{A}_{1}, \mathcal{A}_{2}, \ldots, \mathcal{A}_{N_{1}}$ denote the connected components of $\bigcup_{i \in G_{0}} \overline{\tau_{i}}$. Let

$$
\mathcal{G}_{k}:=\left\{i: \tau_{i} \subset \mathcal{A}_{k}\right\} \text { and define } \widetilde{\mathcal{A}}_{k}:=\bigcup_{\left\{i: \mathbb{V}_{1}(i) \subset \mathcal{G}_{k}\right\}} \overline{\tau_{i}} .
$$

Define

$$
E(z)=\left\{i: \overline{\tau_{i}} \cap B_{\eta}(z) \neq \emptyset\right\} \text { for any } z \in Q_{\epsilon^{-\frac{1}{2}}+\eta}(0) .
$$

Note $\operatorname{Card}(E(z)) \leq c$ and note

$$
E(z) \subset \mathbb{V}_{1}(i) \text { for any } z \text { such that } B_{\frac{3 \eta}{2}}(z) \cap \overline{\tau_{i}} \neq \emptyset \text {. }
$$

Step 1. Given $k \in\left\{1,2, \ldots, N_{1}\right\}$ we will show there exists $k_{0} \in\{1,2, \ldots, N\}$ such that

$$
d\left(D v_{\left\lfloor\tau_{i}\right.}, S O(2) A_{k_{0}}\right)=d\left(D v_{\left\lfloor\tau_{i}\right.}, K\right) \text { for every } i \in \mathcal{G}_{k} .
$$

Proof of Step 1. Suppose this is not true. So we can find $k_{0} \in\left\{1,2, \ldots, N_{1}\right\}$ and some $N_{0} \in\{2,3, \ldots, N\}$ for which we have disjoint subsets $\Omega_{1}, \Omega_{2}, \ldots, \Omega_{N_{0}} \subset \mathcal{G}_{k_{0}}$ with $\bigcup_{i=1}^{N_{0}} \Omega_{i}=\mathcal{G}_{k_{0}}$ and for each $k \in\left\{1,2, \ldots, N_{0}\right\}$ there exists $p_{k} \in\{1,2, \ldots, N\}$ such that

$$
d\left(D v_{\left\lfloor\tau_{i}\right.}, S O(2) A_{p_{k}}\right)=d\left(D v_{\left\lfloor\tau_{i}\right.}, K\right) \text { for all } i \in \Omega_{k} \text { for } k=1,2, \ldots, N_{0} .
$$

Since $\bigcup_{i \in \mathcal{G}_{k_{0}}} \tau_{i}=\mathcal{A}_{k_{0}}$ and $\mathcal{A}_{k_{0}}$ is connected we must be able to find $i_{1} \in \Omega_{1}$ and $i_{2} \in \Omega_{2}$ such that $H^{1}\left(\partial \tau_{i_{1}} \cap \partial \tau_{i_{2}}\right) \geq \varsigma$. Let $a, b$ be the endpoints of $\partial \tau_{i_{1}} \cap \partial \tau_{i_{2}}$, since (by definition of $\left.G_{0}\right) d\left(D v_{\left\lfloor\tau_{i_{1}}\right.}, S O(2) A_{p_{1}}\right) \leq$ $\eta, d\left(D v_{\left\lfloor\tau_{i_{2}}\right.}, S O(2) A_{p_{2}}\right) \leq \eta$ and $D v_{\left\lfloor\tau_{i_{1}}\right.}(a-b)=D v_{\left\lfloor\tau_{i_{2}}\right.}(a-b)$ we must have that for some $R_{1}, R_{2} \in S O(2)$,

$$
\left|R_{1} A_{p_{1}}(a-b)-R_{2} A_{p_{2}}(a-b)\right| \leq 3 \eta
$$

since $u \in \mathcal{D}_{F}^{\varsigma, \sqrt{\epsilon}}$ the edges of the triangles are parallel to $w_{1}, w_{2}$ and $\frac{w_{1}-w_{2}}{\left|w_{1}-w_{2}\right|}$. Thus (assuming $a, b$ are ordered correctly) $\frac{a-b}{|a-b|} \in\left\{w_{1}, w_{2}, \frac{w_{1}-w_{2}}{\left|w_{1}-w_{2}\right|}\right\}$. Recall we chose $w_{1}, w_{2}$ so that $\left\{w_{1}, w_{2}, \frac{w_{1}-w_{2}}{\left|w_{1}-w_{2}\right|}\right\}$ are not in the set of rank-1 connections between $S O(2) A_{p_{1}}$ and $S O(2) A_{p_{2}}$. So $\left|A_{p_{1}}\left(\frac{a-b}{|a-b|}\right)\right| \neq\left|A_{p_{2}}\left(\frac{a-b}{|a-b|}\right)\right|$, we can assume without loss of generality there is a constant $c_{4}=c_{4}\left(w_{1}, w_{2}\right)>1$ such that $\left|A_{p_{1}}\left(\frac{a-b}{|a-b|}\right)\right|>c_{4}\left|A_{p_{2}}\left(\frac{a-b}{|a-b|}\right)\right|$. Assuming we chose $\eta$ small enough this contradicts (5.141) this completes the proof of Step 1.

Step 2. Given $k_{0} \in\left\{1,2, \ldots, N_{1}\right\}$ and $x \in \widetilde{\mathcal{A}}_{k_{0}}$ we will show that

$$
\max \left\{\left|D v_{\left\lfloor\tau_{i}\right.}-D v_{\left\lfloor\tau_{l} \mid\right.}\right|: i, l \in E(x)\right\} \leq c \max \left\{d\left(D v_{\left\lfloor\tau_{j}\right.}, K\right): j \in E(x)\right\} .
$$

Proof Step 2. Firstly by change of variables we can assume $k_{0}$ is such that $D v_{\left\lfloor\tau_{i}\right.} \in N_{\eta}(S O(2))$ for any $i \in G_{0}$. We introduce some notation, let $j \in\left\{1,2, \ldots, N_{3}\right\}$ for any $p \in V(j)$ define

$$
a(j, p):=\max \left\{d\left(D v_{\left\llcorner\tau_{j}\right.}, S O(2)\right), d\left(D v_{\left\llcorner\tau_{p}\right.}, S O(2)\right)\right\}
$$

so there exists $R_{j} \in S O(2), R_{p} \in S O(2)$ such that

$$
\left|D v_{\left\lfloor\tau_{p}\right.}-R_{p}\right| \leq 2 a(j, p),\left|D v_{\left\lfloor\tau_{j}\right.}-R_{j}\right| \leq 2 a(j, p) .
$$




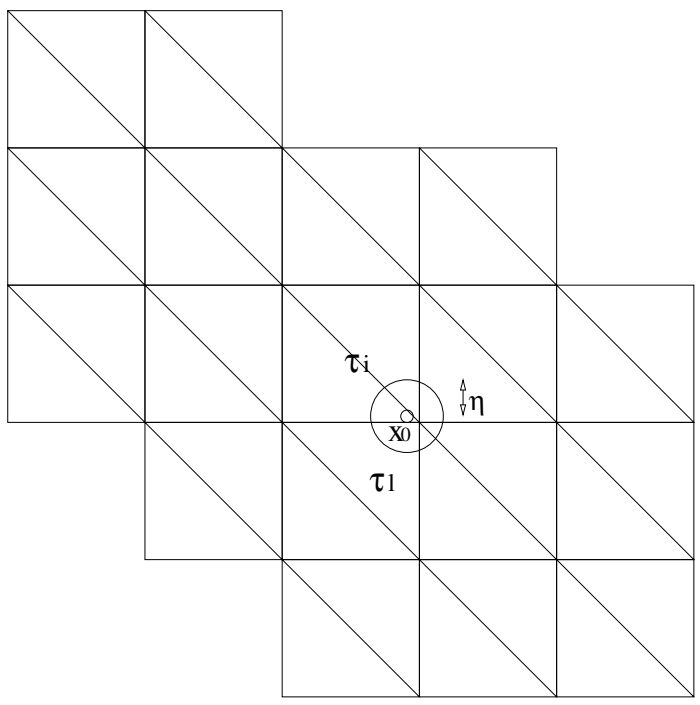

FiguRE 6. A typical ball in the triangulation.

Since $H^{1}\left(\overline{\tau_{p}} \cap \overline{\tau_{j}}\right) \geq \varsigma$, let $a, b$ denote the endpoints of $\overline{\tau_{p}} \cap \overline{\tau_{j}}$, so as $D v_{\left\lfloor\tau_{p}\right.}(a-b)=D v_{\left\lfloor\tau_{j}\right.}(a-b)$ we have $\left|R_{p}(a-b)-R_{j}(a-b)\right| \leq 4 a(j, p)$ which implies $\left|R_{p}-R_{j}\right| \leq 4 \varsigma^{-1} a(j, p)$. Putting this together with (5.143) gives

$$
\left|D v_{\left\lfloor\tau_{p}\right.}-D v_{\left\lfloor\tau_{j}\right.}\right| \leq c a(j, p) .
$$

Pick $i, l \in E(x)$, now (see Fig. 6) we must be able to find ${ }^{7} i_{1}, i_{2}, \ldots, i_{M_{1}} \in E\left(x_{0}\right)$ with the following properties

(1) $i_{0}=i, i_{M_{1}}=l$;

(2) $i_{r+1} \in V\left(i_{r}\right)$ for $r=0,1, \ldots, M_{1}-1$;

(3) $i_{r_{1}} \neq i_{r_{2}}$ for $r_{1} \neq r_{2}$;

(4) $E\left(x_{0}\right) \subset \bigcup_{r=0}^{M_{1}} V\left(i_{r}\right)$.

We have

$$
\begin{aligned}
\left|D v_{\left\lfloor\tau_{i_{0}}\right.}-D v_{\left\lfloor\tau_{i_{M_{1}}}\right.}\right| & \stackrel{(5.144)}{\leq} \sum_{r=0}^{M_{1}-1} c a\left(i_{r}, i_{r+1}\right) \\
& \leq \quad c M_{1} \max \left\{d\left(D v_{\left\lfloor\tau_{r}\right.}, S O(2)\right): r \in E(x)\right\}
\end{aligned}
$$

Since from property (3) we know $M_{1} \leq c \operatorname{Card}\left(E\left(x_{0}\right)\right) \leq c$ this gives (5.142).

Step 3. Let $\tilde{v}:=v * \rho_{\eta}$ we will show

$$
\sum_{k=1}^{N_{1}} \int_{\widetilde{\mathcal{A}}_{k}} d^{p}(D \tilde{v}(z), K) \mathrm{d} L^{2} z \leq c \alpha_{0}
$$

${ }^{7}$ Since $B_{\eta}(x)$ is open and $\tau_{i} \cap B_{\eta}(x) \neq \emptyset, \tau_{l} \cap B_{\eta}(x) \neq \emptyset$ we have $H^{1}\left(\partial B_{\eta}(x) \cap \tau_{i}\right)>0$ and $H^{1}\left(\partial B_{\eta}(x) \cap \tau_{l}\right)>0$. Pick point $s_{0} \in \tau_{i} \cap \partial B_{\eta}(x)$ and a point $s_{M_{1}} \in \tau_{l} \cap \partial B_{\eta}(x)$, since all but finitely many points on $\partial B_{\eta}(x)$ are contained in $\bigcup_{j} \tau_{j}$ we can go clockwise from $s_{1}$ to $s_{M_{1}}$, the first triangle $\tau_{j}$ we encounter after $\tau_{i}$ with $H^{1}\left(\tau_{j} \cap \partial B_{\eta}(x)\right)>0$ will have the property that $\tau_{j} \cap B_{\eta}(x) \neq \emptyset$ (and hence $j \in E\left(x_{0}\right)$ ) and $j \in V_{1}(i)$ so define $i_{1}=j$. We can then define $i_{2}$ to be the first $\tau_{l}$ we encounter going clockwise on $\partial B_{\eta}(x)$ after $\tau_{i_{1}} \cap \partial B_{\eta}(x)$, continuing in this way gives us the sequence $i_{1}, i_{2}, \ldots, i_{M_{1}}$ with the properties we want. 
Proof of Step 3. Let $\mathbb{D}:=\left\{i: \partial \tau_{i} \cap \partial \Omega \neq \emptyset\right\}$. We define $p: Q_{\epsilon^{-\frac{1}{2}}+\eta}(0) \rightarrow\left\{1,2, \ldots, N_{3}\right\}$ by

$$
p(z):= \begin{cases}\min \left\{i: z \in \overline{\tau_{i}}\right\} & \text { for } z \in \overline{\Omega_{\epsilon^{-\frac{1}{2}}}} \\ \min \left\{i \in \mathbb{D}: B_{\frac{3 \eta}{2}}(z) \cap \overline{\tau_{i}} \neq \emptyset\right\} & \text { for } z \in \Omega_{\epsilon^{-\frac{1}{2}}+\eta} \backslash \overline{\epsilon^{-\frac{1}{2}}}\end{cases}
$$

Fix $k_{0} \in\left\{1,2, \ldots, N_{1}\right\}$, assume $\widetilde{\mathcal{A}}_{k_{0}} \neq \emptyset$. Let $y \in \widetilde{\mathcal{A}}_{k_{0}}$. Pick $i_{0} \in E(y)$ and let $R_{0} \in K$ be such that $d\left(D v_{\left\lfloor\tau_{i_{0}}\right.}, K\right)=\left|D v_{\left\lfloor\tau_{i_{0}}\right.}-R_{0}\right|$. Now

$$
\begin{aligned}
\left|D \tilde{v}(y)-R_{0}\right| & =\left|\sum_{j \in E(y)} \int_{\tau_{j}}\left(D v_{\left\lfloor\tau_{j}\right.}(x)-R_{0}\right) \rho_{\eta}(x-y) \mathrm{d} L^{2} x\right| \\
& \leq \quad c \sum_{j \in E(y)}\left|D v_{\left\lfloor\tau_{j}\right.}-D v_{\left\lfloor\tau_{\tau_{0}}\right.}\right|+\left|D v_{\left\lfloor\tau_{i_{0}}\right.}-R_{0}\right| \\
\stackrel{(5.142)}{\leq} & c \max \left\{d\left(D v_{\left\lfloor\tau_{j}\right.}, K\right): j \in E(y)\right\} .
\end{aligned}
$$

Define $c(i) \in \mathbb{V}_{1}(i)$ to be such that

$$
d\left(D v_{\left\lfloor\tau_{c(i)}\right.}, K\right)=\max \left\{d\left(D v_{\left\lfloor\tau_{j}\right.}, K\right): j \in \mathbb{V}_{1}(i)\right\}
$$

Note for any $z \in Q_{\epsilon^{-\frac{1}{2}}+\eta}(0)$ from (5.139) we know (recall definition (5.138)) that $E(y) \subset \mathbb{V}_{1}(p(y)$ ), so

$$
d^{p}(D \tilde{v}(y), K) \stackrel{(5.147),(5.148)}{\leq} c d^{p}\left(D v_{\left\lfloor\tau_{c(p(y))}\right.}, K\right)
$$

Now

$$
\begin{aligned}
& \int_{\widetilde{\mathcal{A}}_{k_{0}}} d^{p}(D \tilde{v}(z), K) \mathrm{d} L^{2} z \leq \sum_{\left\{i: \mathbb{V}_{1}(i) \subset \mathcal{G}_{k_{0}}\right\}} L^{2}\left(\tau_{i}\right) \sup \left\{d^{p}(D \tilde{v}(z), K): z \in \tau_{i}\right\} \\
& \stackrel{(5.149)}{\leq} \sum_{\left\{i: \mathbb{V}_{1}(i) \subset \mathcal{G}_{k_{0}}\right\}} c d^{p}\left(D v_{\tau_{c(i)}}, K\right)
\end{aligned}
$$

Note $\max \left\{\operatorname{Card}\left(c^{-1}(i)\right): i \in \mathcal{G}_{k_{0}}\right\} \leq c$ and so $\int_{\widetilde{\mathcal{A}}_{k_{0}}} d^{p}(D \tilde{v}(z), K) \mathrm{d} L^{2} z \leq c \sum_{i \in \mathcal{G}_{k_{0}}} d^{p}\left(D v_{\left\lfloor\tau_{i}\right.}, K\right)$. Thus summing over $k_{0}=1,2, \ldots, N_{1}$ gives (5.145).

Step 4. We will show that

$$
\int_{Q_{\epsilon^{-\frac{1}{2}+\eta}}(0)} d^{p}(D \tilde{v}(z), K) \mathrm{d} L^{2} z \leq c \alpha_{0}+c \eta \epsilon^{-\frac{1}{2}}
$$

Proof of Step 4 . Let $\mathbb{D}:=\left\{i: \partial \tau_{i} \cap \partial \Omega \neq \emptyset\right\}$. Note (recalling definition (5.146), (5.138))

$$
p(z) \in E(z) \text { for any } z \in \Omega_{\epsilon^{-\frac{1}{2}}}
$$


SO

$$
\begin{aligned}
&|D \tilde{v}(z)|=\left|F \int_{B_{\eta}(z) \backslash \Omega_{\epsilon}-\frac{1}{2}} \rho_{\eta}(a-z) \mathrm{d} L^{2} a+\sum_{i \in E(z)} D v_{\left\lfloor\tau_{i}\right.} \int_{\tau_{i}} \rho_{\eta}(a-z) \mathrm{d} L^{2} a\right| \\
& \stackrel{(5.139)}{\leq} c+c \sum_{i \in \mathbb{V}_{1}(p(z))} d\left(D v_{\left\lfloor\tau_{i}\right.}, K\right) .
\end{aligned}
$$

Thus

$$
\begin{aligned}
d^{p}(D \tilde{v}(z), K) & \stackrel{(5.152)}{\leq}\left(c+c \sum_{i \in \mathbb{V}_{1}(p(z))} d\left(D v_{\left\lfloor\tau_{i}\right.}, K\right)\right)^{p}+c \\
& \leq c+c \sum_{i \in \mathbb{V}_{1}(p(z))} d^{p}\left(D v_{\left\lfloor\tau_{i}\right.}, K\right) .
\end{aligned}
$$

Let $\mathbb{B}:=\left\{i: \mathbb{V}_{1}(i) \not \subset G_{0}\right\}$. Note that if $i$ is such that $\mathbb{V}_{1}(i) \subset G_{0}$ then $\mathbb{V}_{1}(i) \subset \mathcal{G}_{k}$ for some $k \in\left\{1,2, \ldots, N_{1}\right\}$ (and recall definition (5.137)) and hence $\tau_{i} \subset \widetilde{\mathcal{A}}_{k}$, thus

$$
\bigcup_{i \in \mathbb{B}} \overline{\tau_{i}}=\overline{\Omega_{\epsilon^{-\frac{1}{2}}} \backslash\left(\bigcup_{k=1}^{N_{1}} \widetilde{\mathcal{A}}_{k}\right)} .
$$

So

$$
\begin{gathered}
\int_{\bigcup_{i \in \mathbb{B}} \overline{\tau_{i}}} d^{p}(D \tilde{v}(z), K) \mathrm{d} L^{2} z \stackrel{(5.153)}{\leq} \sum_{i \in \mathbb{B}} L^{2}\left(\tau_{i}\right)\left(c+c \sum_{j \in \mathbb{V}_{1}(i)} d^{p}\left(D v_{\left\lfloor\tau_{j}\right.}, K\right)\right) \\
\stackrel{(5.136)}{\leq} c \alpha_{0}+c \operatorname{Card}(\mathbb{B}) .
\end{gathered}
$$

By an easy application of the $5 r$ Covering Theorem (Thm. 2.1. [25]) we know

$$
\operatorname{Card}(\mathbb{B}) \leq c\left(\left\{1,2, \ldots, N_{3}\right\} \backslash G_{0}\right) \leq c \alpha_{0}
$$

Now

$$
\tau_{p(z)} \subset \Omega_{\epsilon^{-\frac{1}{2}}} \backslash \Omega_{\epsilon^{-\frac{1}{2}}-10 \varsigma^{-1}} \text { for any } z \in \Omega_{\epsilon^{-\frac{1}{2}}+\eta} \backslash \Omega_{\epsilon^{-\frac{1}{2}}}
$$

Let $\left\{l_{1}, l_{2}, \ldots, l_{X_{1}}\right\}$ be an ordering of the set $\left\{p(z): z \in \Omega_{\epsilon^{-\frac{1}{2}}+\eta} \backslash \Omega_{\epsilon^{-\frac{1}{2}}}\right\}$ we have that $X_{1} \leq c \epsilon^{-\frac{1}{2}}$. And thus

$$
\begin{gathered}
\int_{\Omega_{\epsilon^{-\frac{1}{2}}+\eta} \backslash \Omega_{\epsilon^{-\frac{1}{2}}}} d^{p}(D \tilde{v}(z), K) \mathrm{d} L^{2} z \stackrel{(5.153)}{\leq} c \sum_{k=1}^{X_{1}} L^{2}\left(p^{-1}\left(l_{k}\right) \backslash \Omega_{\epsilon^{-\frac{1}{2}}}\right)+\sum_{k=1}^{X_{1}} \sum_{i \in \mathbb{V}_{1}\left(l_{k}\right)} c d^{p}\left(D v_{\left\lfloor\tau_{i}\right.}, K\right) \\
\stackrel{(5.136)}{\leq} c \eta \epsilon^{-\frac{1}{2}}+c \alpha_{0}
\end{gathered}
$$


So putting things together, by $(5.145),(5.154),(5.155),(5.156)$ and $(5.158)$ we have

$$
\begin{aligned}
\int_{\Omega_{\epsilon}-\frac{1}{2}+\eta} d^{p}(D \tilde{v}(z), K) \mathrm{d} L^{2} z= & \int_{\bigcup_{i \in \mathbb{B}} \tau_{i}} d^{p}(D \tilde{v}(z), K) \mathrm{d} L^{2} z \\
& +\int_{\bigcup_{k}^{N_{1}} \tilde{\mathcal{A}}_{k}} d^{p}(D \tilde{v}(z), K) \mathrm{d} L^{2} z \\
& +\int_{\Omega_{\epsilon^{-\frac{1}{2}}+\eta}(0) \backslash \Omega_{\epsilon} e^{-\frac{1}{2}}} d^{p}(D \tilde{v}(z), K) \mathrm{d} L^{2} z \\
\leq & c \alpha_{0}+c \eta \epsilon^{-\frac{1}{2}},
\end{aligned}
$$

which completes the proof of (5.150).

Step 5. We will show

$$
\sum_{k=1}^{N_{1}} \int_{\widetilde{A}_{k}}\left|D^{2} \tilde{v}(y)\right|^{2} \mathrm{~d} L^{2} y \leq c \alpha_{0}
$$

Proof of Step 5. Let $y \in \bigcup_{k=1}^{N_{1}} \widetilde{A}_{k}$, for each $j \in E(y)$ define $A_{j}:=\int_{\tau_{j}} D \rho_{\eta}(x-y) \mathrm{d} L^{2} x$, note $\sum_{j \in E(y)} A_{j}=0$. So $D^{2} \tilde{v}(y)=\sum_{j \in E(y)} \int_{\tau_{j}}-D v_{\left\lfloor\tau_{j}\right.} \otimes D \rho_{\eta}(x-y) \mathrm{d} L^{2} x=\sum_{j \in E(y)}-D v_{\left\lfloor\tau_{j}\right.} \otimes A_{j}$. So we have $D^{2} \tilde{v}(y)=$ $\sum_{j \in E(y)}-\left(D v_{\left\lfloor\tau_{j}\right.}-D v_{\left\lfloor\tau_{p(y)}\right)}\right) \otimes A_{j}$ and so

$$
\begin{aligned}
\left|D^{2} \tilde{v}(y)\right|^{2} & \leq \quad c \sum_{j \in E(y)}\left|D v_{\left\lfloor\tau_{j}\right.}-D v_{\left\lfloor\tau_{p(y)}\right.}\right|^{2} \\
& \stackrel{(5.142),(5.151)}{\leq} c\left(\operatorname { m a x } \left\{d \left(D v_{\left.\left.\left\lfloor\tau_{l}, K\right): l \in E(y)\right\}\right)^{2} .}\right.\right.\right.
\end{aligned}
$$

Thus (recall the definition $c(i),(5.148))$ we have

$$
\begin{aligned}
& \int_{\widetilde{A}_{k}}\left|D^{2} \tilde{v}(y)\right|^{2} \mathrm{~d} L^{2} y \quad \stackrel{(5.139),(5.161)}{\leq} \sum_{\left\{i: \mathbb{V}_{1}(i) \subset \mathcal{G}_{k}\right\}} c\left(\max \left\{d\left(D v_{\left\lfloor\tau_{l}\right.}, K\right): l \in \mathbb{V}_{1}(i)\right\}\right)^{2} \\
& =\quad \sum_{\left\{i: \mathbb{V}_{1}(i) \subset \mathcal{G}_{k}\right\}} c d^{2}\left(D v_{\left\lfloor\tau_{c(i)}, K\right.}, K\right) \\
& \leq \quad c \sum_{i \in \mathcal{G}_{k}} d^{p}\left(D v_{\left\lfloor\tau_{i}\right.}, K\right) .
\end{aligned}
$$

Thus summing over $k=1,2, \ldots, N_{1}$ gives (5.160).

Step 6. We will show

$$
\int_{\Omega_{\epsilon}-\frac{1}{2}+\eta} \backslash\left(\cup_{k=1}^{N_{1}} \widetilde{\mathcal{A}}_{k}\right)\left|D^{2} \tilde{v}(z)\right|^{2} \mathrm{~d} L^{2} z \leq c \alpha_{0}+c \eta \epsilon^{-\frac{1}{2}} .
$$

Proof of Step 6. Now let $y \in \Omega_{\epsilon^{-\frac{1}{2}}+\eta}$. Note that if $B_{\eta}(y) \not \subset \Omega_{\epsilon^{-\frac{1}{2}}}$ then define $A_{y}:=\int_{B_{\eta}(y) \backslash \Omega_{\epsilon^{-\frac{1}{2}}}} D \rho_{\eta}(x-y) \mathrm{d} L^{2} x$ otherwise define $A_{y}=0$.

As in Step 5 for each $j \in E(y)$ define $A_{j}=\int_{\tau_{j}} D \rho_{\eta}(x-y) \mathrm{d} L^{2} x$. So we have $\sum_{j \in E(y)} A_{j}+A_{y}=0$. So as in Step $5-D^{2} \tilde{v}(y)=F \otimes A_{y}+\sum_{j \in E(y)} D v_{\left\lfloor\tau_{j}\right.} \otimes A_{j}=\left(F-D v_{\left\lfloor\tau_{p(y)}\right.}\right) \otimes A_{y}+\sum_{j \in E(y)}\left(D v_{\left\lfloor\tau_{j}\right.}-D v_{\left\lfloor\tau_{p(y)}\right.}\right) \otimes A_{j}$. 
Thus for any $y \in Q_{\epsilon^{-\frac{1}{2}}+\eta}(0)$

$$
\begin{aligned}
\left|D^{2} \tilde{v}(y)\right|^{2} & \leq c\left|F-D v_{\left\lfloor\tau_{p(y)} \mid\right.}\right|^{2}\left|A_{y}\right|^{2}+c \sum_{j \in E(y)}\left|D v_{\left\lfloor\tau_{j}\right.}-D v_{\left\lfloor\tau_{p(y)}\right.}\right|^{2} \\
\stackrel{(5.139)}{\leq} & c\left|F-D v_{\left\lfloor\tau_{p(y)} \mid\right.}\right|^{2}\left|A_{y}\right|^{2}+c \sum_{j \in \mathbb{V}_{1}(p(y))}\left|D v_{\left\lfloor\tau_{j}\right.}-D v_{\left\lfloor\tau_{p(y)}\right.}\right|^{2} .
\end{aligned}
$$

Now as in Step 1 for any $i, j \in \mathbb{V}_{1}(p(y))$ we can find a finite sequence $l_{1}, l_{2}, \ldots, l_{N_{j}} \in \mathbb{V}_{1}(p(y))$ such that $l_{1}=i, l_{a+1} \in V\left(l_{a}\right)$ for $a=1,2, \ldots, N_{j}-1$ and $l_{N_{j}}=j$ so

$$
\begin{aligned}
\left|D v_{\left\lfloor\tau_{i}\right.}-D v_{\left\lfloor\tau_{j} \mid\right.}\right|^{2} & \leq c \sum_{a=1}^{N_{j}-1}\left|D v_{\left\lfloor\tau_{l_{a+1}}\right.}-D v_{\left\lfloor\tau_{l a}\right.}\right|^{2} \\
& \leq c \sum_{l \in\left\{l_{1}, l_{2}, \ldots, l_{N_{j}-1}\right\}} \sum_{k \in V(l)}\left|D v_{\left\lfloor\tau_{l}\right.}-D v_{\left\lfloor\tau_{k}\right.}\right|^{2} \\
& \leq c \sum_{l \in \mathbb{V}_{1}(p(y))} \sum_{k \in V(l)}\left|D v_{\left\lfloor\tau_{l}\right.}-D v_{\left\lfloor\tau_{k}\right.}\right|^{2} .
\end{aligned}
$$

So from (5.163) for any $y \in Q_{\epsilon^{-\frac{1}{2}}+\eta}(0)$ we have

$$
\begin{aligned}
\left|D^{2} \tilde{v}(y)\right|^{2} & \leq c\left|F-D v_{\left\lfloor\tau_{p(y)}\right.}\right|^{2}\left|A_{y}\right|^{2}+c \sum_{l \in \mathbb{V}_{1}(p(y))} \sum_{k \in V(l)}\left|D v_{\left\lfloor\tau_{l}\right.}-D v_{\left\lfloor\tau_{k}\right.}\right|^{2} \\
& \leq c\left|F-D v_{\left\lfloor\tau_{p(y)}\right.}\right|^{2}\left|A_{y}\right|^{2}+c \sum_{l \in \mathbb{V}_{1}(p(y)) \cap J(v)} \sum_{k \in V(l)}\left|D v_{\left\lfloor\tau_{l}\right.}-D v_{\left\lfloor\tau_{k}\right.}\right|^{2}+c .
\end{aligned}
$$

Recall $\mathbb{D}=\left\{i: \partial \tau_{i} \cap \partial \Omega_{\epsilon^{-\frac{1}{2}}} \neq \emptyset\right\}$. Note if $y \in \bigcup_{i \notin \mathbb{D}} \overline{\tau_{i}}$ then $B_{\eta}(y) \subset \Omega_{\epsilon^{-\frac{1}{2}}}$ and so $A_{y}=0$. For $i \in \mathbb{B}$ let $y_{i} \in \overline{\tau_{i}}$ be such that $\left|D^{2} \tilde{v}\left(y_{i}\right)\right|=\sup \left\{\left|D^{2} \tilde{v}(y)\right|: y \in \tau_{i}\right\}$, thus

$$
\begin{aligned}
& \int_{\Omega_{\epsilon^{-\frac{1}{2}}} \backslash\left(\cup_{k=1}^{N_{1}} \widetilde{\mathcal{A}}_{k}\right)}\left|D^{2} \tilde{v}(y)\right|^{2} \mathrm{~d} L^{2} y \stackrel{(5.154)}{\leq} \sum_{i \in \mathbb{B}} L^{2}\left(\tau_{i}\right)\left|D^{2} \tilde{v}\left(y_{i}\right)\right|^{2} \\
& \stackrel{(5.164)}{\leq} c \sum_{i \in \mathbb{B} \backslash \mathbb{D}} \sum_{l \in \mathbb{V}_{1}(i) \cap J(v)} \sum_{k \in V(l)}\left|D v_{\left\lfloor\tau_{l}\right.}-D v_{\left\lfloor\tau_{k}\right.}\right|^{2} \\
& +c \sum_{i \in \mathbb{B} \cap \mathbb{D}}\left(\left|F-D v_{\left\lfloor\tau_{i}\right.}\right|^{2}\left|A_{y_{i}}\right|^{2}+\sum_{l \in \mathbb{V}_{1}(i) \cap J(v)} \sum_{k \in V(l)}\left|D v_{\left\lfloor\tau_{l}\right.}-D v_{\left\lfloor\tau_{k}\right.}\right|^{2}\right)+c \operatorname{Card}(\mathbb{B}) \\
& \leq c \sum_{i \in \mathbb{B}} \sum_{l \in \mathbb{V}_{1}(i) \cap J(v)} \sum_{k \in V(l)}\left|D v_{\left\lfloor\tau_{l}\right.}-D v_{\left\lfloor\tau_{k}\right.}\right|^{2}+c \sum_{i \in \mathbb{B} \cap \mathbb{D}}\left|F-D v_{\left\lfloor\tau_{i}\right.}\right|^{2}+c \operatorname{Card}(\mathbb{B}) \\
& \leq c \sum_{l \in J(v)} \sum_{k \in V(l)}\left|D v_{\left\lfloor\tau_{l}\right.}-D v_{\left\lfloor\tau_{k}\right.}\right|^{2}+c \sum_{i \in \mathbb{D}}\left|F-D v_{\left\lfloor\tau_{i}\right.}\right|^{2}+c \operatorname{Card}(\mathbb{B}) \\
& \stackrel{(5.156)}{\leq} c \sum_{l \in J(v)} \sum_{M \in N(l)}\left|D v_{\left\lfloor\tau_{l}\right.}-M\right|^{2}+c \alpha_{0} \\
& \stackrel{(5.135),(5.136)}{\leq} c \alpha_{0}
\end{aligned}
$$


Now to estimate $\left.\int_{\Omega_{\epsilon^{-\frac{1}{2}}+\eta}}\left|\Omega_{\epsilon^{-\frac{1}{2}}}\right| D^{2} \tilde{v}(z)\right|^{2} \mathrm{~d} L^{2} z$ we argue as in Step 3, let $\left\{l_{1}, l_{2}, \ldots, l_{X_{1}}\right\}$ be an ordering of the set $\left\{p(z): z \in \Omega_{\epsilon^{-\frac{1}{2}}+\eta} \backslash \Omega_{\epsilon^{-\frac{1}{2}}}\right\}$, recall we have $X_{1} \leq c \epsilon^{-\frac{1}{2}}$. And of course, from (5.146) we have $\left\{l_{1}, l_{2}, \ldots, l_{X_{1}}\right\} \subset \mathbb{D}$. So

$$
\begin{aligned}
\int_{\Omega_{\epsilon^{-\frac{1}{2}}+\eta}(0) \backslash \Omega_{\epsilon}-\frac{1}{2}}\left|D^{2} \tilde{v}(z)\right|^{2} \mathrm{~d} L^{2} z \stackrel{(5.164)}{\leq} & \sum_{a=1}^{X_{1}} c\left|F-D v_{\left\lfloor\tau_{l a}\right.}\right|^{2}+c \sum_{l \in \mathbb{V}_{1}\left(l_{a}\right) \cap J(v)} \sum_{k \in V(l)}\left|D v_{\left\lfloor\tau_{l}\right.}-D v_{\left\lfloor\tau_{k}\right.}\right|^{2} \\
& +c \sum_{b=1}^{X_{1}} c L^{2}\left(p^{-1}\left(l_{b}\right)\right) \\
\leq & c \sum_{l=1}^{N_{3}} \sum_{k \in V(l)}\left|D v_{\left\lfloor\tau_{l}\right.}-D v_{\left\lfloor\tau_{k}\right.}\right|^{2}+c \sum_{i \in \mathbb{D}}\left|F-D v_{\left\lfloor\tau_{i}\right.}\right|^{2}+c \eta \epsilon^{-\frac{1}{2}} \\
& (5.135) \\
\leq & c \int_{\Omega} d^{p}(D v(z), K) \mathrm{d} L^{2} z+c \eta \epsilon^{-\frac{1}{2}} \\
\leq & c \alpha_{0}+c \eta \epsilon^{-\frac{1}{2}}
\end{aligned}
$$

Putting this together with (5.165) gives (5.162).

Proof of Proposition 5.2. Let $w(z):=\frac{\tilde{v}\left(\left(\epsilon^{-\frac{1}{2}}+\eta\right) z\right)}{\epsilon^{-\frac{1}{2}}+\eta}$, it is clear $w$ can also be defined by equation (5.133). So from (5.162) and (5.160) we have

$$
\int_{\Omega}\left|D^{2} w(z)\right|^{2} \mathrm{~d} L^{2} z \leq c \alpha_{0}+c \eta \epsilon^{-\frac{1}{2}}
$$

and

$$
\begin{aligned}
& \int_{\Omega} d^{p}(D w(z), K) \mathrm{d} L^{2} z \quad=\quad \int_{\Omega_{\epsilon^{-\frac{1}{2}}+\eta}} d^{p}(D \tilde{v}(y), K)\left(\epsilon^{-\frac{1}{2}}+\eta\right)^{-2} \mathrm{~d} L^{2} y \\
& \stackrel{(5.150)}{\leq} \quad c \epsilon \alpha_{0}+c \eta \epsilon^{\frac{1}{2}}
\end{aligned}
$$

Putting this together with (5.166) gives

$$
\int_{\Omega} d^{p}(D w(z), K)+\epsilon\left|D^{2} w(z)\right|^{2} \mathrm{~d} L^{2} z \leq c \epsilon \alpha_{0}+c \eta \epsilon^{\frac{1}{2}}
$$

Now by (4.2) we have that there exists some small constant $c_{1}=c_{1}(\sigma)$ such that

$$
c_{1} \epsilon^{\frac{1}{2}} \leq \int_{\Omega} d^{p}(D w(z), K)+\epsilon\left|D^{2} w(z)\right|^{2} \mathrm{~d} L^{2} z
$$

so assuming we have chosen $\eta$ small enough we have that

$$
\int_{\Omega} d^{p}(D w(z), K)+\epsilon\left|D^{2} w(z)\right|^{2} \mathrm{~d} L^{2} z-c \eta \epsilon^{\frac{1}{2}} \geq \frac{1}{2} \int_{\Omega} d^{p}(D w(z), K)+\epsilon\left|D^{2} w(z)\right|^{2} \mathrm{~d} L^{2} z
$$


hence from (5.168) we have

$$
\begin{aligned}
& \int_{\Omega} d^{p}(D w(z), K)+\epsilon\left|D^{2} w(z)\right|^{2} \mathrm{~d} L^{2} z \leq \quad c \epsilon \alpha_{0} \\
& \stackrel{(5.136)}{=} c \int_{\Omega} d^{p}(D w(z), K) \mathrm{d} L^{2} z
\end{aligned}
$$

which completes the proof of (5.134).

\subsection{The proof of Theorem 1.1 completed}

By Proposition 5.2 for any $\epsilon>0$ we can find $u \in \mathcal{D}_{F}^{\varsigma, \sqrt{\epsilon}}$ such that $\int_{\Omega} d^{p}(D u(z), K) \mathrm{d} L^{2} z \leq c m_{\epsilon}^{p}$ which obviously implies there must exist constant $\mathcal{C}_{1}<1$ such that $\mathcal{C}_{1} \alpha(\sqrt{\epsilon}) \leq m_{\epsilon}^{p}$.

Let $u \in \mathcal{D}_{F}^{\varsigma, \sqrt{\epsilon}}$ be such that $\int_{\Omega} d^{p}(D u(z), K) \mathrm{d} L^{2} z \leq c \alpha_{p}(\sqrt{\epsilon})$. By Proposition 5.3 function $w$ defined by (5.132) and (5.133) has the property that

$$
I_{\epsilon}(w) \leq c \int_{\Omega} d^{p}(D u(z), K) \mathrm{d} L^{2} z \leq c \alpha_{p}(\sqrt{\epsilon})
$$

which implies there exists a constant $\mathcal{C}_{2}>1$ such that $m_{\epsilon}^{p} \leq \mathcal{C}_{2} \alpha_{p}(\sqrt{\epsilon})$.

Acknowledgements. Firstly we would like to thank Georg Dolzmann for pointing out the connection between finite element approximations and problems which constrain surface energy. In some sense this paper is nothing more than a precise formulation of this observation. Secondly we owe a great debt to discussions with Stefan Müller, particularly with reference to Proposition 5.1. Suggestions from him lead to a cleaner statement and a drastic simplification of proof. Finally we thank the referee for some very helpful and accurate comments.

\section{REFERENCES}

[1] L. Ambrosio, A. Coscia and G. Dal Maso, Fine properties of functions with bounded deformation. Arch. Rational Mech. Anal. 139 (1997) 201-238.

[2] L. Ambrosio, N. Fusco and D. Pallara, Functions of bounded variation and free discontinuity problems, Oxford Mathematical Monographs. The Clarendon Press, Oxford University Press, New York (2000).

[3] J.M. Ball and R.D. James, Fine phase mixtures as minimisers of energy. Arch. Rational Mech. Anal. 100 (1987) 13-52.

[4] J.M. Ball and R.D. James, Proposed experimental tests of a theory of fine microstructure and the two well problem. Phil. Trans. Roy. Soc. London Ser. A 338 (1992) 389-450.

[5] M. Chipot, The appearance of microstructures in problems with incompatible wells and their numerical approach. Numer. Math. 83 (1999) 325-352.

[6] M. Chipot and D. Kinderlehrer, Equilibrium configurations of crystals. Arch. Rational Mech. Anal. 103 (1988) $237-277$.

[7] M. Chipot and S. Müller, Sharp energy estimates for finite element approximations of non-convex problems, in Variations of domain and free-boundary problems in solid mechanics (Paris, 1997), Solid Mech. Appl. 66, Kluwer Acad. Publ., Dordrecht (1999) 317-325.

[8] S. Conti, Branched microstructures: scaling and asymptotic self-similarity. Comm. Pure Appl. Math. 53 (2000) $1448-1474$.

[9] S. Conti and B. Schweizer, Rigidity and Gamma convergence for solid-solid phase transitions with $S O(2)$-invariance. Comm. Pure Appl. Math. 59 (2006) 830-868.

[10] S. Conti, D. Faraco and F. Maggi, A new approach to counterexamples to $L^{1}$ estimates: Korn's inequality, geometric rigidity, and regularity for gradients of separately convex functions. Arch. Rational Mech. Anal. 175 (2005) 287-300.

[11] S. Conti, G. Dolzmann and B. Kirchheim, Existence of Lipschitz minimizers for the three-well problem in solid-solid phase transitions. Ann. Inst. H. Poincaré Anal. Non Linéaire 24 (2007) 953-962.

[12] B. Dacorogna and P. Marcellini, General existence theorems for Hamilton-Jacobi equations in the scalar and vectorial cases. Acta Math. 178 (1997) 1-37.

[13] C. De Lellis and L. Székelyhidi, The Euler equations as a differential inclusion. Ann. Math. (to appear).

[14] G. Dolzmann and K. Bhattacharya, Relaxed constitutive relations for phase transforming materials. The J. R. Willis 60 th anniversary volume. J. Mech. Phys. Solids 48 (2000) 1493-1517. 
[15] G. Dolzmann and B. Kirchheim, Liquid-like behavior of shape memory alloys. C. R. Math. Acad. Sci. Paris 336 (2003) 441-446.

[16] G. Dolzmann and S. Müller, Microstructures with finite surface energy: the two-well problem. Arch. Rational Mech. Anal. 132 (1995) 101-141.

[17] L.C. Evans and R.F. Gariepy, Measure theory and fine properties of functions, Studies in Advanced Mathematics. CRC Press, Boca Raton, FL (1992).

[18] G. Friesecke, R.D. James and S. Müller, A theorem on geometric rigidity and the derivation of nonlinear plate theory from three dimensional elasticity. Comm. Pure Appl. Math. 55 (2002) 1461-1506.

[19] B. Kirchheim, Deformations with finitely many gradients and stability of quasiconvex hulls. C. R. Acad. Sci. Paris Sér. I Math. 332 (2001) 289-294.

[20] B. Kirchheim, Rigidity and Geometry of Microstructures. Lectures note 16/2003, Max Planck Institute for Mathematics in the Sciences, Leipzig (2003).

[21] R.V. Kohn, New Estimates for Deformations in Terms of Their Strains. Ph.D. thesis, Princeton University, USA (1979).

[22] R.V. Kohn and S. Müller, Surface energy and microstructure in coherent phase transitions. Comm. Pure Appl. Math. 47 (1994) 405-435.

[23] A. Lorent, An optimal scaling law for finite element approximations of a variational problem with non-trivial microstructure. ESAIM: M2AN 35 (2001) 921-934.

[24] M. Luskin, On the computation of crystalline microstructure. Acta Numer. 5 (1996) 191-257.

[25] P. Mattila, Geometry of Sets and Measures in Euclidean Spaces, Cambridge Studies in Advanced Mathematics 44. Cambridge University Press (1995).

[26] S. Müller, Singular perturbations as a selection criterion for periodic minimizing sequences. Calc. Var. Partial Differ. Equ. 1 (1993) 169-204.

[27] S. Müller, Variational models for microstructure and phase transitions, in Calculus of variations and geometric evolution problems (Cetraro, 1996), Lecture Notes in Mathematics 1713, Springer, Berlin (1999) 85-210. www.mis.mpg.de/cgi-bin/lecturenotes.pl.

[28] S. Müller, Uniform Lipschitz estimates for extremals of singularly perturbed nonconvex functionals. MIS MPG, Preprint 2 (1999).

[29] S. Müller and V. Šverák, Attainment results for the two-well problem by convex integration, in Geometric Analysis and the Calculus of Variations. For Stefan Hildebrandt, J. Jost Ed., International Press, Cambridge (1996) 239-251.

[30] S. Müller and V. Šverák, Convex integration with constraints and applications to phase transitions and partial differential equations. J. Eur. Math. Soc. (JEMS) 1 (1999) 393-422.

[31] S. Müller and V. Šverák, Convex integration for Lipschitz mappings and counterexamples to regularity. Ann. Math. 157 (2003) $715-742$.

[32] S. Müller, M. Rieger and V. Šverák, Parabolic systems with nowhere smooth solutions. Arch. Rational Mech. Anal. 177 (2005) $1-20$.

[33] M.A. Sychev, Comparing two methods of resolving homogeneous differential inclusions. Calc. Var. Partial Differ. Equ. 13 (2001) 213-229.

[34] M.A. Sychev and S. Müller, Optimal existence theorems for nonhomogeneous differential inclusions. J. Funct. Anal. 181 (2001) $447-475$.

[35] V. Šverák, On the problem of two wells, in Microstructure and phase transition, D. Kinderlehrer, R.D. James, M. Luskin and J. Ericksen Eds., IMA Vol. Math. Appl. 54, Springer, New York (1993) 183-189. 